Florida International University FIU Digital Commons

$7-3-2003$

\title{
Static and dynamic mechanical testing of a polymer with potential use as heart valve material
}

Andres Felipe Aguirre

Florida International University

DOI: $10.25148 /$ etd.FI13101544

Follow this and additional works at: https://digitalcommons.fiu.edu/etd

Part of the Biomedical Engineering and Bioengineering Commons

\section{Recommended Citation}

Aguirre, Andres Felipe, "Static and dynamic mechanical testing of a polymer with potential use as heart valve material" (2003). FIU Electronic Theses and Dissertations. 1162.

https://digitalcommons.fiu.edu/etd/1162 
FLORIDA INTERNATIONAL UNIVERSITY

Miami, Florida

STATIC AND DYNAMIC MECHANICAL TESTING OF A POLYMER WITH POTENTIAL USE AS HEART VALVE MATERIAL

A thesis submitted in partial fulfillment of the

requirements for the degree of

MASTER OF SCIENCE

in

BIOMEDICAL ENGINEERING

by

Andrés Felipe Aguirre 
To: Dean Vish Prasad

College of Engineering

This thesis, written by Andrés Felipe Aguirre, and entitled Static and Dynamic Mechanical Testing of a Polymer with Potential Use as Heart Valve Material, having been approved in respect to style and intellectual content, is referred to you for judgment.

We have read this thesis and recommend that it be approved.

James E. Moore Jr.

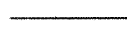

$\overline{\text { Arvind Agarwal }}$

Richard T. Schoephoerster, Major Professor

Date of Defense: July 3, 2003

The thesis of Andrés Felipe Aguirre is approved.

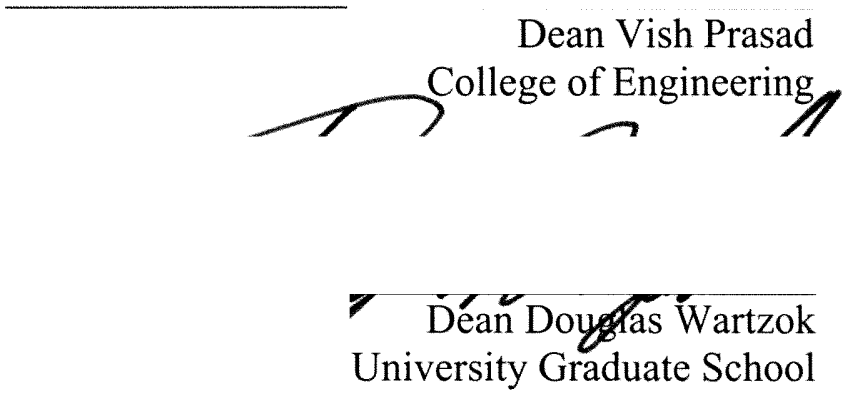

Florida International University, 2003 


\section{DEDICATION}

I dedicate this thesis to my Family and to Maria. Their love and support has been crucial in order for me to complete this work. 


\section{ACKNOWLEDGMENTS}

Two and a half years ago I knocked on Dr. Schoephoerster's door. I told him I wanted to work towards a master's degree under his supervision. He told me he had nothing available at the time. Four more visits after that he had something for me. Dr. Schoephoerster, thank you for giving me a chance and believing in me.

Maria Marquine, thank you for your love, hope, and understanding. Your help with the document was very important.

Mariana Oliva, thank you for your long hours of work making the specimens for my tests.

Siobhain Gallocher, thank you for always being there for me.

Dr. Vladimir Kasyanov, I really enjoyed working with you. Thank you for your support.

Dr. James Byrne, your help in the lab was very valuable.

Valve Team, thank you for creating such an enjoyable work environment.

Dr. Moore and Dr. Agarwal, thank you for your guidance and advice.

Dr. Wu and his team, thank you for your help and patience.

Ivan Erazo, thank you for being my friend and helping me during the rough times. 


\section{ABSTRACT OF THE THESIS}

\section{STATIC AND DYNAMIC MECHANICAL TESTING OF A POLYMER WITH \\ POTENTIAL USE AS HEART VALVE MATERIAL}

by

Andrés Felipe Aguirre

Florida International University, 2003

Miami, Florida

Professor Richard T. Schoephoerster, Major Professor

Synthetic tri-leaflet heart valves generally fail in the long-term use (more than 10 years). Tearing and calcification of the leaflets usually cause failure of these valves as a consequence of high tensile and bending stresses borne on the material. The primary purpose of this study was to explore the possibilities of a new polymer composite to be used as synthetic tri-leaflet heart valve material. This composite was comprised of polystyrene-polyisobutylene-polystyrene (Quatromer), a proprietary polymer, embedded with continuous polypropylene (PP) fibers. Quatromer had been found to be less likely to degrade in vivo than polyurethane. Moreover, it was postulated that a decrease in tears and perforations might result from fiber-reinforced leaflets reducing high stresses on the leaflets. The static and dynamic mechanical properties of the Quatromer/PP composite were compared with those of an implant-approved polyurethane (PU) for cardiovascular applications. Results show that the reinforcement of Quatromer with PP fibers improves both its static and dynamic properties as compared to the PU. Hence, this composite has the potential to be a more suitable material for synthetic tri-leaflet heart valves. 


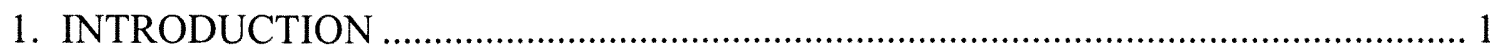

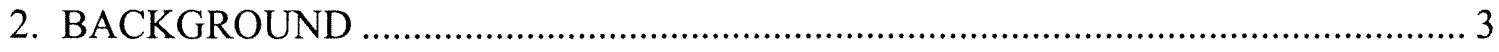

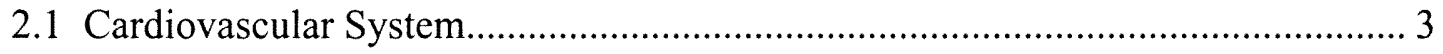

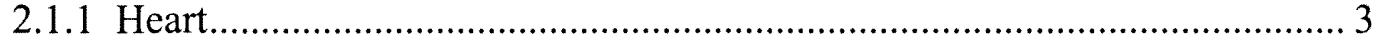

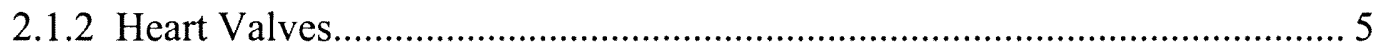

2.1.3 Heart Valve Diseases ................................................................................ 7

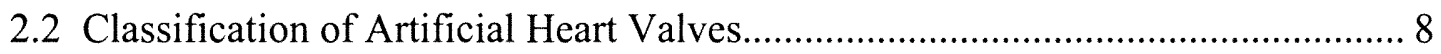

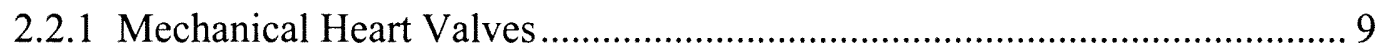

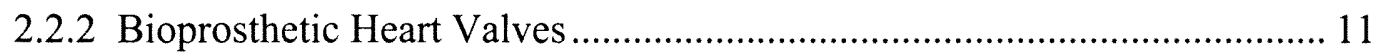

2.2.3 Synthetic Tri-leaflet Heart Valves .......................................................... 12

2.3 Polymers in Synthetic Tri-leaflet Heart Valves ................................................... 13

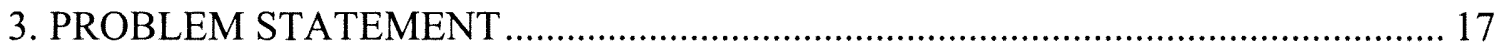

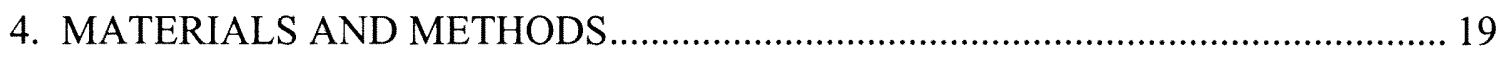

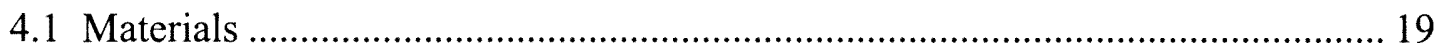

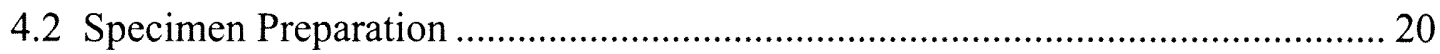

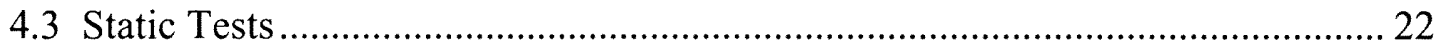

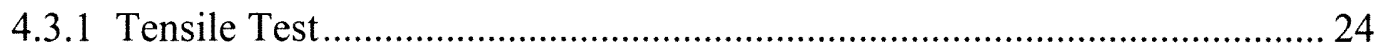

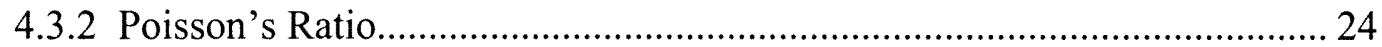

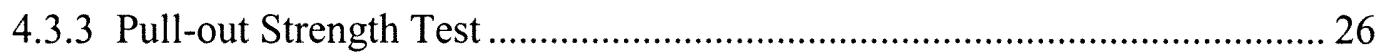

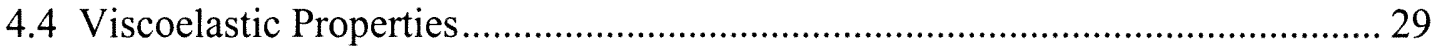

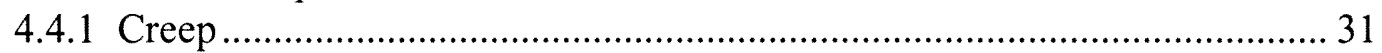

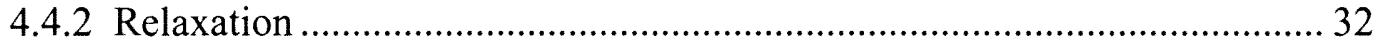

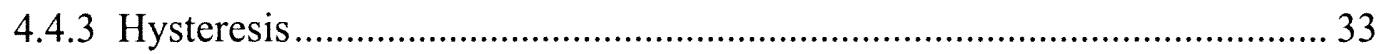

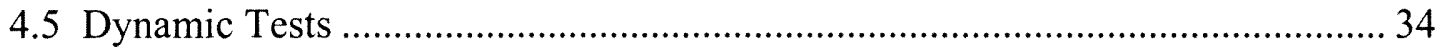

4.5.1 Tension-Tension Fatigue Test....................................................... 35

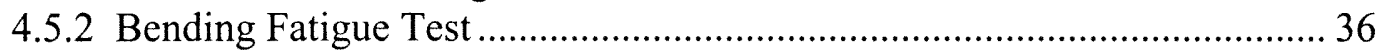

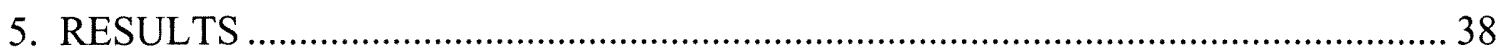

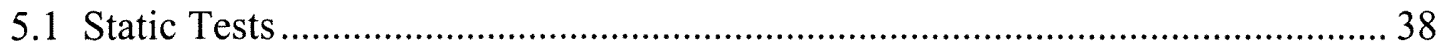

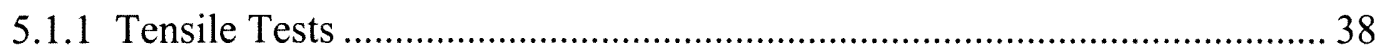

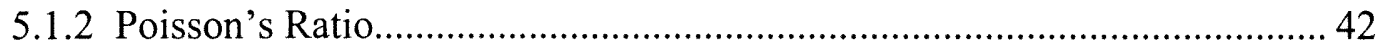

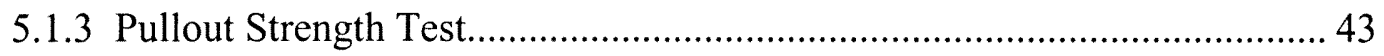

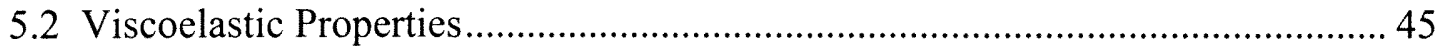

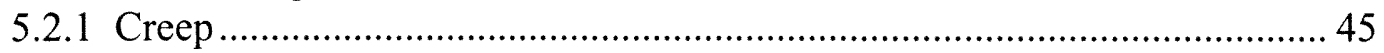

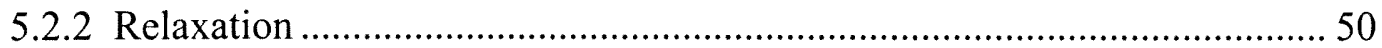

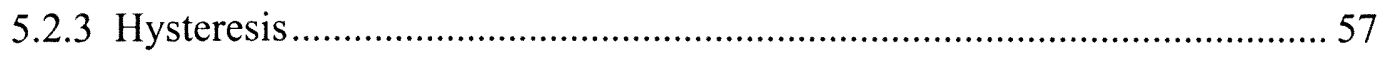

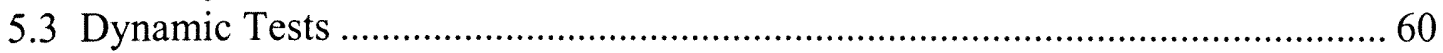

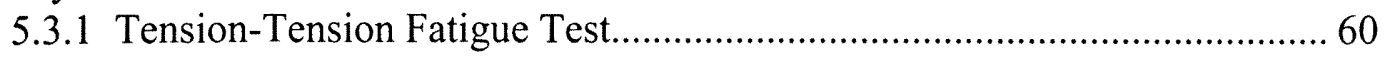




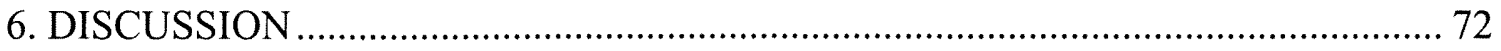

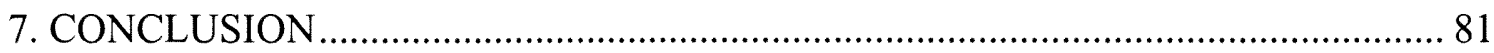

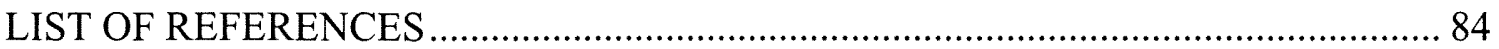




\section{LIST OF TABLES}

TABLE

PAGE

1. Number of years equivalent to bending cycles 37

2. Young's modulus (E), Ultimate Strain (US), Ultimate Tensile Stress (UTS), and Number of specimens tested $(\mathrm{N})$ of each material.

3. Values for $A_{f}, A_{c}, E_{f}$, and $E_{m}$ 41

4. Volume fraction of the fibers $\left(\mathrm{V}_{\mathrm{f}}\right)$, and theoretical (rule of mixture) and experimental values of the composite's Young's modulus $\left(\mathrm{E}_{\mathrm{c}}\right)$.

5. Strain $(\varepsilon)$ and Poisson's Ratio measurements of Quatromer ( $\varepsilon$ units are in $\mathrm{mm} / \mathrm{mm}$ ) . 43

6. Numerical summary of the creep test 47

7. Numerical summary of the creep test 48

8. ANOVA for $\tau_{\varepsilon}$ (CSTRAIN) and $\tau_{\sigma}$ (CSTRESS) 49

9. Tukey post hoc test for $\tau_{\varepsilon}$. 49

10. Tukey post hoc test for $\tau_{\sigma}$ 50

11. Numerical summary of the relaxation test

12. Numerical summary of the relaxation test a) upper $63.2 \%$ of the curve, b) lower $36.8 \%$ of the curve.

13. ANOVA for $\tau_{\varepsilon}$ (CSTRAIN) and $\tau_{\sigma}$ (CSTRESS); a) upper $63.2 \%$, b) lower $36.8 \%$ of the curve

14. Tukey post hoc test for $\tau_{\varepsilon}$ (upper $63.2 \%$ of the curve,). Mean values are shown. ...... 56

15. Tukey post hoc test for $\tau_{\sigma}$ (upper $63.2 \%$ of the curve). Mean values are shown........ 57

16. Numerical summary of the tensile results of the bending fatigue test a) Quatromer, b) $\mathrm{Q}+12$ PP Fibers 63

17. One-way ANOVA results for all Quatromer groups 65

18. Tukey post hoc test for the True Young's modulus ( $\left.E_{t r u e}\right)$ of Quatromer. 66 
19. Tukey post hoc test for the Engineering Young's modulus $\left(E_{\text {eng }}\right)$ of Quatromer ....... 66

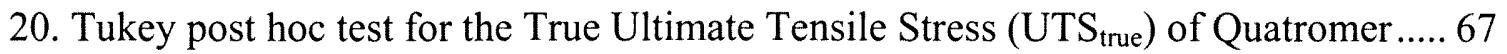

21. Tukey post hoc test for the Engineering Ultimate Tensile Stress $\left(\mathrm{UTS}_{\text {eng }}\right)$ of Quatromer.

22. One-way ANOVA results for all Quatromer + 12 PP Fibers groups ........................... 68

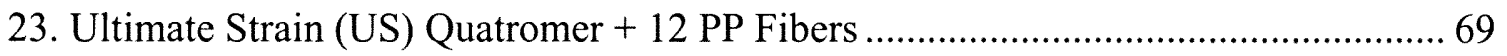

24. True Young's Modulus $\left(\mathrm{E}_{\text {true }}\right)$ for Quatromer +12 PP Fibers .....................................6 69

25. True Ultimate Tensile Stress $\left(\mathrm{UTS}_{\text {true }}\right)$ for Quatromer + 12 PP Fibers........................ 70

26. True Young's Modulus $\left(\mathrm{E}_{\text {eng }}\right)$ for Quatromer + 12 PP Fibers ….............................. 70

27. True Ultimate Tensile Stress $\left(\mathrm{UTS}_{\text {eng }}\right)$ for Quatromer + 12 PP Fibers ....................... 71

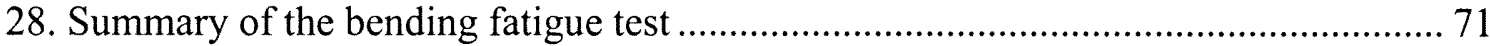


FIGURE

1. A diagrammatic frontal section of the heart, showing major landmarks and the path of blood (marked by arrows) ....................................................................................... 4

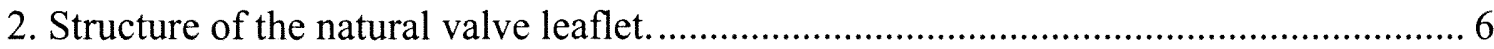

3. Natural valve leaflet. The clear lines are collagen fiber networks............................... 7

4. Five Types Of Prosthetic Heart Valves: ……........................................................... 10

5. Three-plate assembly used to mold rectangular specimen ......................................... 20

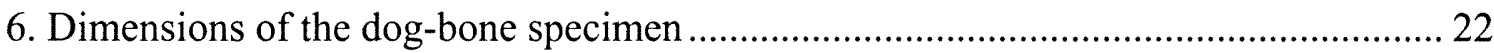

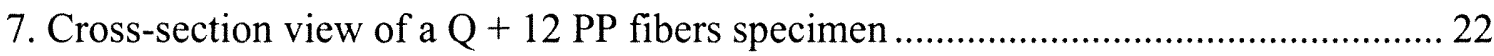

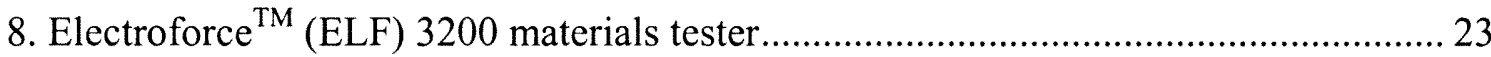

9. Quatromer specimen mounted on the testing machine showing the location of the four

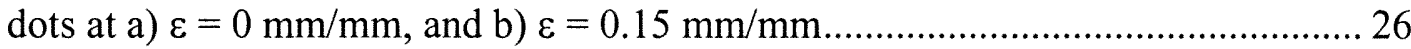

10. Specimen of Quatromer with 1 PP fiber embedded..................................................... 27

11. Specimen of Quatromer with 1 PP fiber embedded. The specimen mounted on the

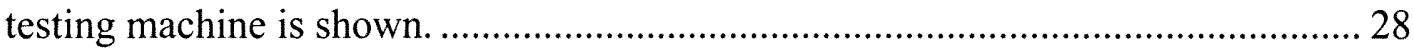

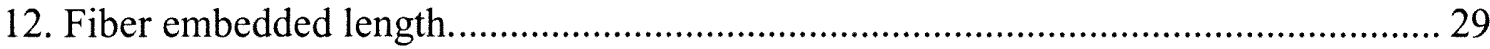

13. Kelvin Model o Standard linear solid model ............................................................ 30

14. Creep function of a Kelvin Model or Standard linear solid model........................... 32

15. Relaxation function of a Kelvin Model o Standard linear solid model ..................... 33

16. Measurement of the curvatures produced in the bending fatigue test. ...................... 37

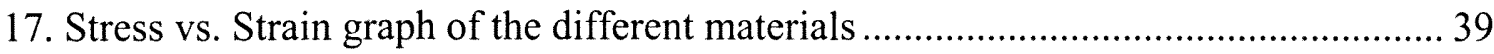

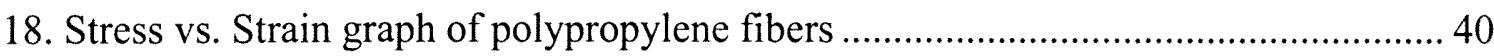

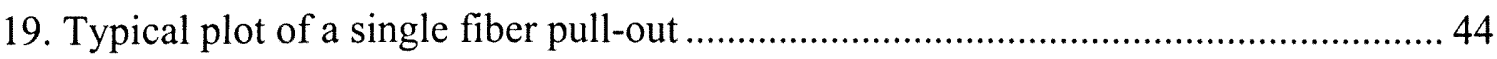

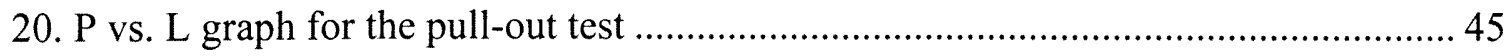


21. Plot of creep tests. a) Strain vs. time, b) normalized strain vs. time 46

22. Normalized stress vs. time creep test results (initial 10 seconds) 46

23. Normalized stress relaxation plotted against time 51

24. Normalized stress relaxation plotted against time (first 20 seconds) 52

25. Normalized stress relaxation plotted against time (Last 80 seconds) 52

26. Hysteresis test results at $1.25 \mathrm{~Hz}$ 58

27. Hysteresis test results at $1.25 \mathrm{~Hz}$ 58

28. Normalized hysteresis test results

29. The dynamic modulus of elasticity $(|\mathrm{G}|)$ and the internal damping $(\tan \delta)$ vs. logarithm of frequency $(\omega)$ 60

30. Stress vs. Number of Cycles of the different materials on a semi-log scale. Arrows indicate the material did not fail after 350 million cycles

31. Stress vs. Number of Cycles of the different materials. Arrows indicate the material did not fail after 350 million cycles 


\section{INTRODUCTION}

Since the 1960's heart valve prostheses have been efficiently used in helping patients with heart disease improve their overall quality of life (Edmunds 2001). Not only have heart valve prostheses extended life, but have also lessened the symptoms due to valvular heart disease. Nevertheless, as reported by NIH's Working Group on Heart Valves, 10-year mortality rates range from $30-55 \%$, indicating that advancements in valve design are still necessary. Moreover, efforts need to be directed toward improving morbidity and mortality outcomes, and should focus on minimizing structural degradation and thrombotic potential (Schoephoerster and Chandran 1991).

Currently, there are three kinds of heart valve prostheses: mechanical, bioprosthetic, and flexible membrane tri-leaflet made from synthetic resinous materials. Mechanical valves generally show excellent durability, but also require most patients to receive permanent anticoagulant therapy due to thrombotic reactions (Schoephoerster and Chandran 1991; Cannegieter, Rosendaal et al. 1995). Bioprosthetic valves exhibit advantages in hemodynamic properties, producing the central flow characteristic of natural valves (Chandran, Fatemi et al. 1989). However, they also show leaflet stiffening due to mineralization, which results in short fatigue life (usually less than 10 years) (Senthilnathana, Treasurea et al. 1999). Synthetic tri-leaflet valves are fabricated from biochemically inert synthetic materials, with polyurethane the typical material of choice (Schoephoerster, Gallocher et al. 2001). These valves present natural hemodynamics while also having the potential for long-term durability. Unfortunately, they have not been successful to date due to long-term material degradation. Long-term material 
degradation occurs through a combination of oxidative reactions with blood and the high dynamic tensile and bending stresses borne by the material. These valves show stress concentrations in the leaflets (Imamura and Kaye 1977). This, along with the thickness of the leaflet material, is an important factor limiting the long-term function of polymer valve prostheses (Reul 1983; Bernacca, O'Connor et al. 2002). Moreover, it was postulated that a decrease in tears and perforations might result from fiber-reinforced leaflets reducing high stresses on the leaflets.

The primary purpose of this thesis was to explore the possibilities of a new polymer composite to be used as a flexible membrane trileaflet heart valve material. This composite is composed of polystyrene-polyisobutylene-polystyrene (Quatromer), a proprietary polymer, embedded with continuous $10-0$ monofilament $(25 \mu \mathrm{m}$ diameter) polypropylene fibers (Prolene, Ethicon). Quatromer has been found to be less likely to degrade in vivo than polyurethane (Pinchuk, Khan et al. 1999). Hence, a Quatromer/polypropylene composite has the potential to become the material of choice for flexible membrane trileaflet heart valves. Polypropylene fibers have several unique properties that make them especially suited for use as leaflet reinforcement in a synthetic tri-leaflet heart valve. In order to determine the feasibility of the Quatromer/polypropylene composite as heart valve material, its static and dynamic mechanical properties were compared with those of a polyurethane approved for cardiovascular applications. 


\section{BACKGROUND}

This chapter serves as an introduction to the background on heart valves. The first section is a brief introduction to the cardiovascular system. In this section the heart is presented along with the heart valves and the diseases it most commonly suffers. The second section is an introduction to artificial heart valves. The history and evolution of the different types of heart valves are described as well as advantages and disadvantages of each type. The third section of this chapter is an introduction to polymers and its mechanical characterization.

\subsection{Cardiovascular System}

The cardiovascular system consists of the heart and blood vessels, which work together to supply blood to all the tissues in the body. The heart pumps blood throughout the body through the blood vessels. The blood vessels can be subdivided into the pulmonary circuit and the systemic circuit. The pulmonary circuit carries blood to and from the gas exchange surfaces of the lung, and the systemic circuit transports blood to and from the rest of the body.

\subsubsection{Heart}

The heart is a four-chambered pump approximately the size of a clenched fist and weights just about one pound. In normal conditions it pumps about 5 liters every minute. It is located near the anterior chest wall, directly posterior to the sternum (Martini 2001). 
The four chambers of the heart, in order of blood flow, are the right atrium, right ventricle, left atrium, and left ventricle (Figure 1). The atriums receive blood that is coming to the heart. The right atrium receives deoxygenated blood from the systemic circuit, while the left atrium receives blood from the pulmonary circuit that is rich in oxygen. The ventricles receive blood from the atriums. The right ventricle pumps blood to the lungs where carbon dioxide is removed and blood is refreshed with oxygen. The left ventricle pumps oxygenated blood to the body to provide oxygen and nutrients and to remove waste products.

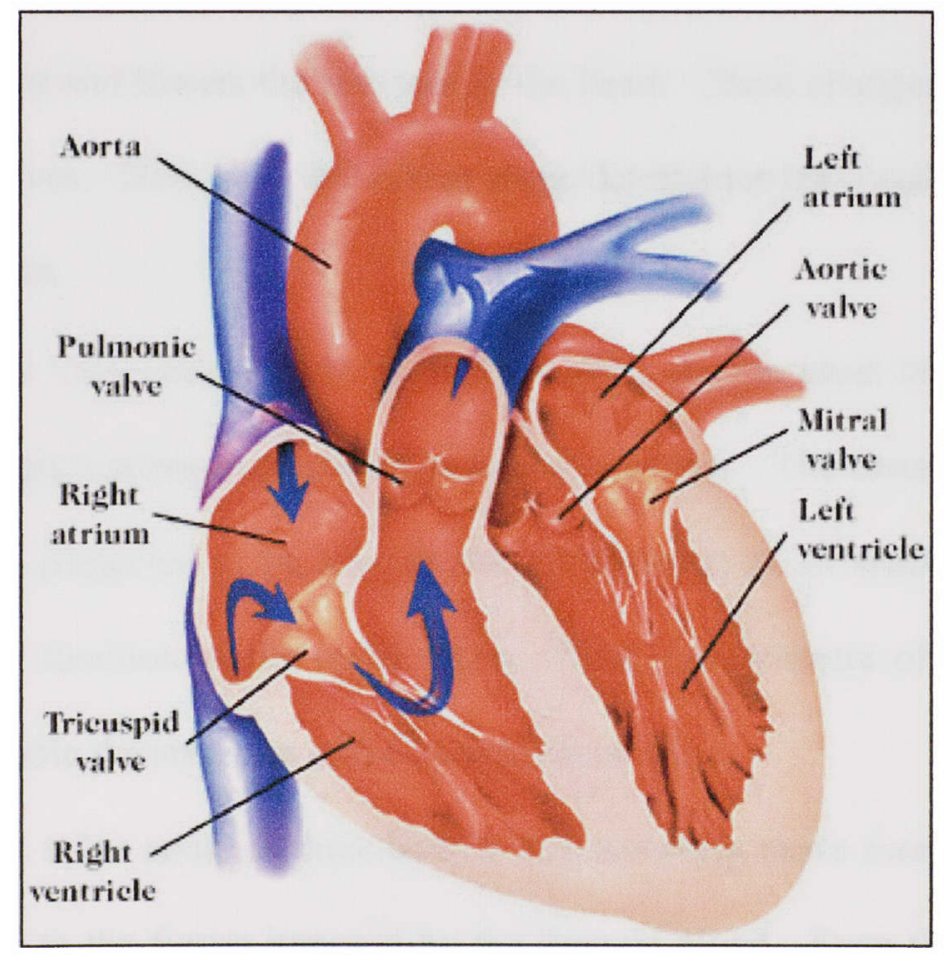

Figure 1. A diagrammatic frontal section of the heart, showing major landmarks and the path of blood (marked by arrows) http://www.cts.usc.edu/hpg-heartvalvesurgery.html 


\subsubsection{Heart Valves}

There are four valves in the heart, one at the exit of each chamber. In order of blood flow they are the tricuspid (right atrium), pulmonary (right ventricle), mitral (left atrium), and aortic (left ventricle) (Figure 1).

The Tricuspid and Mitral valves open during diastole, which is the period of time when the ventricles are filling with blood. The Pulmonic and Aortic valves open during systole, when blood is being ejected from the heart. The purpose of the valves is to make sure that blood flows only in one direction. That is, when the heart squeezes, blood flows in the proper direction, not backwards. During each heartbeat the heart squeezes and relaxes. This rises and lowers the pressure in the heart. These changes in pressure open and close the valves. How well the valves work determines how easily blood flows in and out of the heart.

The aortic valve along with the mitral valve, are the most affected by disease because of the higher-pressure gradients in the left ventricle. The material that we tested in our study was projected to be used in the construction of an artificial valve, which would mimic the function of the aortic valve. Thus, the structure of the material was intended to resemble the structure of the natural aortic valve.

The aortic valve contains three leaflets that passively move away or towards each other in response to the forces imposed by the flow of blood. Even though $90 \%$ of the leaflet is water, it also contains other components, such as connective tissue proteins collagen and elastin, glycosaminoglycans (GAGs, long chain sugars) and a small population of cells. Collagen and elastin are the main structural components, which are mostly responsible for giving the leaflet its unique mechanical properties. In cross- 
section, each leaflet has three distinct layers: the fibrosa, spongiosa, and ventricularis (Figure 2). Looking down from the aorta the fibrosa is on the top surface of the leaflet and the ventricularis is on the bottom. The fibrosa is the primary structural layer and consists mainly of collagen fiber bundles oriented in a circumferential direction, as shown in Figure 3. Hence, the structure of the leaflet is considerably stiffer in the circumferential direction as compared to the radial. The collagen bundles are surrounded by elastin, which helps maintain the valve's microstructure during unloading. The ventricularis is also mainly composed by collagen and elastin, but it is less stiff than the fibrosa because the collagen bundles are not oriented in a specific direction. The spongiosa is primarily water and also contains GAGs and some collagen and elastin that connect the other two layers together. For a more detailed description of the natural aortic histology see Kasyanov et al. (1984).

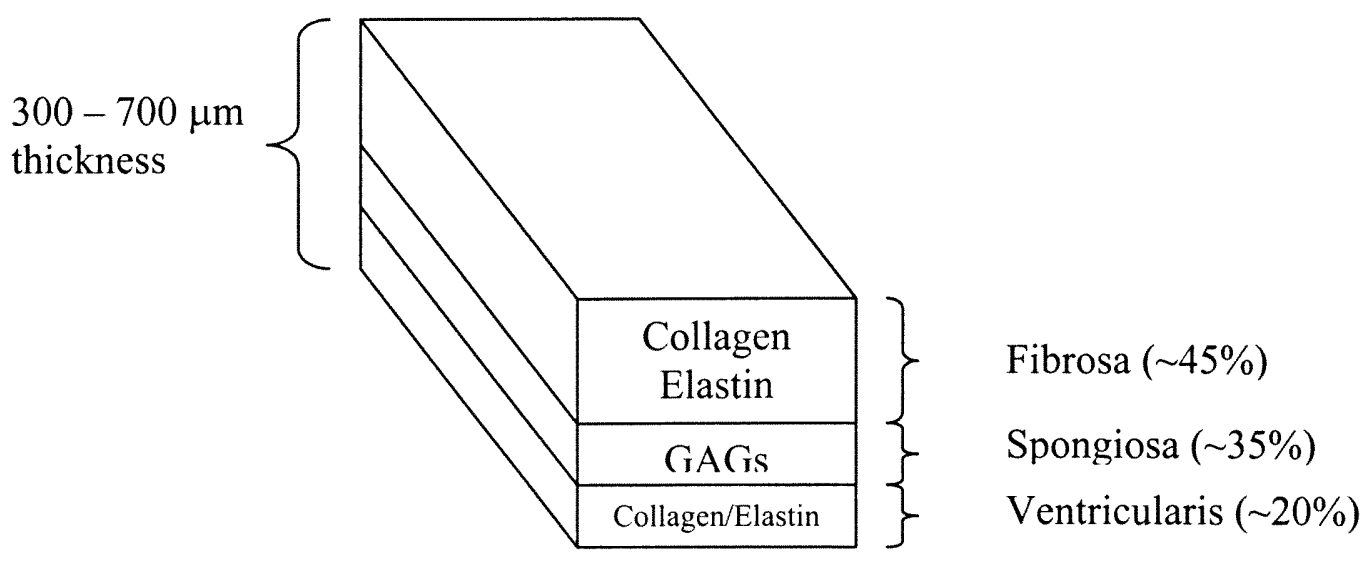

Figure 2. Structure of the natural valve leaflet. 


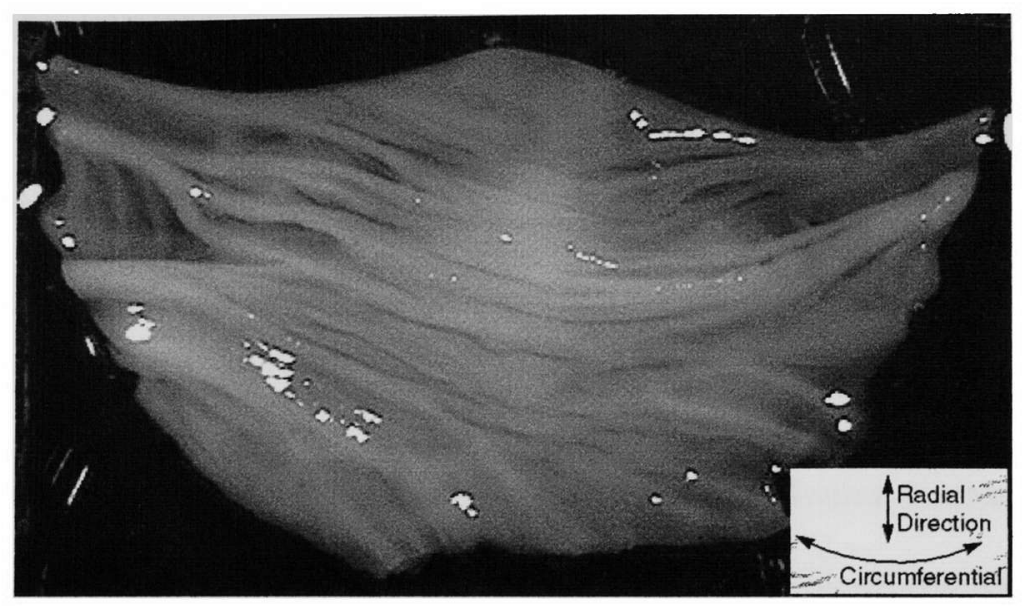

Figure 3. Natural valve leaflet. The clear lines are collagen fiber networks. www.lerner.ccf.org/bme/valve/ images/cusp_25k.jpg

\subsubsection{Heart Valve Diseases}

The functioning of natural heart valves may be deleteriously affected in several ways. A person can be born with an abnormal heart valve. Also, a heart valve can become damaged by infections such as rheumatic fever and infective endocarditis or due to changes in valve structure as a result of aging. As previously mentioned, the most commonly affected valves are the mitral and the aortic valve (Martini 2001).

Disease can affect heart valves in two major ways, stenosis and regurgitation. Stenosis is a condition in which the valve becomes stiff and the aperture is narrowed. When this occurs to the aortic valve the opening through which the blood must flow may become too small and the pressure in the ventricle is increased. When this condition is minor there are no apparent effects, but when this condition is severe it can be life threatening. A valve can become stenotic due to rheumatic fever disease or by age related calcification of the previously normal heart valve. This second condition is more 
common in older people, while the first condition is most common in younger people (Cannegieter, Rosendaal et al. 1995).

Regurgitation is a condition in which the valve allows blood to flow back. When this occurs the heart has to pump more blood than normal in order to carry the normal blood flow to the body. Regurgitation may be caused by a number of diseases such as chronic hypertension that dilates the aortic root, rheumatic valvular disease, chest trauma, infection of the heart valve (endocarditis), certain congenital disorders, and autoimmune diseases (Martini 2001). Again, if this condition is mild there are minimal effects on the overall health of the person. On the other hand, if this condition becomes severe the left ventricle may enlarge in order to maintain its high pumping volume or cardiac output. If this enlargement goes too far permanent damage may occur and replacement of the valve can become necessary (Martini 2001).

\subsection{Classification of Artificial Heart Valves}

Replacement of diseased natural heart valves with artificial ones has been life saving. Artificial heart valves have clinically been used for nearly 4 decades. Currently, in the United States surgical correction of valvular disease accounts for more than 85,000 open-heart operations annually (Sapirstein and Smith 2001). Yet, replacement valves are never as good as healthy natural valves (Senthilnathana, Treasurea et al. 1999). There are three major types of prosthetic heart valves: mechanical, bioprosthetic, and synthetic trileaflet. As described below each has its own advantages and disadvantages. 


\subsubsection{Mechanical Heart Valves}

The primary benefit of mechanical heart valves is that structural failure of approved valves is almost nonexistent, and valves can reasonably be expected to perform properly for at least 20 years. The mechanical performance of these valves is excellent (Sapirstein and Smith 2001). However, the major drawback of using these valves is the need for anticoagulation therapy. The complications of thrombosis, embolism and bleeding contribute significantly to morbidity and mortality (Senthilnathana, Treasurea et al. 1999). The United States Food and Drug Administration (FDA) has approved devices that represent three mechanical valve designs: caged-ball, tilting-disc, and bileaflet.

The Starr-Edwards valve is an example of a caged-ball prosthesis. It was designed in the 1950's and first used clinically in the 1960's (Figure 4a). The fundamental design of this type of valve has not changed over three decades of use. It consists of an occluding ball that moves in a surrounding cage. The cage and ball of the Starr-Edwards heart valve were originally made of methacrylate. Later the methacrylate ball was replaced with a silicone rubber coated nylon ball to reduce valve noise. Alloys of cobalt, chromium, molybdenum, and nickel were used for the cage; and were proved to be biocompatible. Even though this valve was proved to do the job, the presence of the ball in the center of the valve orifice results in considerable turbulent flow and anticoagulation requirements are somewhat higher than for newer mechanical valves. Currently, this valve is rarely used (DeWall, Qasim et al. 2000). 


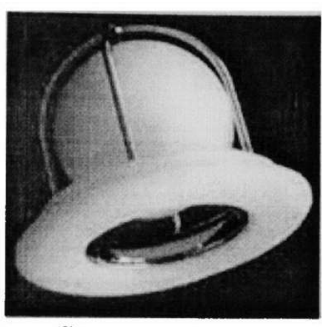

a
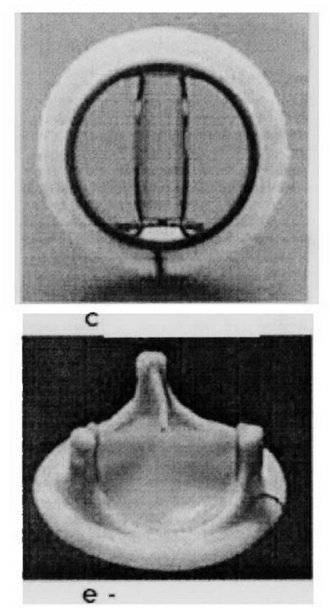

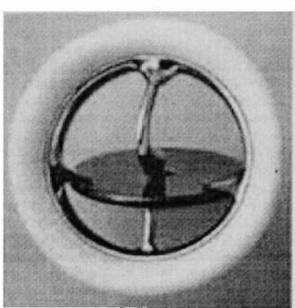

b

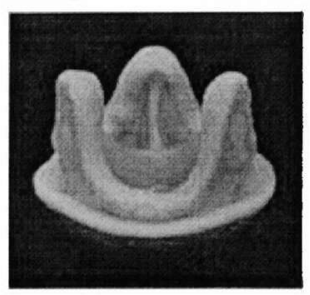

d
Figure 4. Five Types Of Prosthetic Heart

Valves:

(a) Starr-Edwards mitral caged ball valve

(b) Medtronic Hall tilting disk valve

(c) St. Jude bileaflet valve

(d) Hancock porcine valve

(e) Carpentier-Edwards bovine pericardial valve http://www.rjmatthewsmd.com/Definitions /pop/107fig.htm

In the 1970's, the Bjork-Shiley tilting-disc valve became available. This valve consists of a free-floating disc tilting on the edge of an orifice ring (Figure 4b). Two struts arching across the inflow and outflow sides of valve orifice permitted the disc to pivot between open and closed positions. The cage of these valves was made of titanium, while the disc was made of Pyrolyte (DeWall, Qasim et al. 2000). In some instances, with older models, the strut completely separated from the valve ring, and catastrophic disc embolization occurred. These older models were withdrawn from the domestic market in 1988 (DeWall, Qasim et al. 2000).

The most recently approved mechanical heart valve is the St Jude bileaflet valve (Figure 4c). It contains two semicircular discs of pyrolytic carbon, which open with 
independent hinge mechanisms. These valves have profiles that facilitate implantation into small cavities. Opening angles of the leaflets approach 90 degrees, improving valve hemodynamics. The St Jude bileaflet valve is now the most commonly implanted mechanical heart valve (DeWall, Qasim et al. 2000).

\subsubsection{Bioprosthetic Heart Valves}

Tissue valves have been clinically available since the early 1960's when Ross and Barratt-Boyes first described aortic valve replacement with a homograft (Sapirstein and Smith 2001). The primary benefit of this kind of valves is the decreased thrombogenicity compared with a mechanical device. Thus, they do not require long-term anticoagulation therapy. They have excellent hemodynamics, with nonturbulent flow and low transvalvular gradients. Also, they function as quietly as a natural valve. Nevertheless, the major drawback of bioprosthetic valves is the relatively high rate of structural degradation that almost uniformly occurs. They begin to deteriorate after 5 to 6 years of implantation (Fann and Miller 1996). Leaflet wear, worsen by calcification, leads to tears and loss of adequate coaptation. Moreover, they are not suitable for children. Three types of tissue valves are available: porcine xenograft (with and without stent), bovine pericardial valve, and aortic homograft.

The Carpentier-Edwards and Hancock standard porcine heterografts have been available since the 1970's. They have been described as first-generation bioprosthetics. These aortic valves are fixed in glutaraldehyde at high pressure (Figure 4d). 
The Ionescu-Shiley bovine pericardial valve was designed in the 1970's, but its implantation was halted because the device showed evidence of accelerated deterioration. The Carpentier-Edwards Pericardial valve has a better external/internal diameter ratio and a low resistance, which allows improved hemodynamic performance (Figure 4e). The construction is independent of the anatomical constraints imposed by the porcine valves.

Homografts and autografts are the closest to natural valves. A homograft is a graft of tissue obtained from a donor of the same species, but with a different genetic make-up from, the recipient, as a tissue transplant between two humans. An autograft is a tissue or organ grafted into a new position in or on the body of the same individual. Unfortunately, these are technically more difficult to insert, availability is a problem, and optimum preservation is unknown (Fann and Miller 1996).

\subsubsection{Synthetic Tri-leaflet Heart Valves}

The search for a durable, non-thrombogenic valve has led to the consideration of flexible membrane tri-leaflet valves. So far, mechanical valves cannot be matched for durability, and deterioration is the major problem with tissue valves. This third group of

prosthetic heart valves tries to combine the best of both worlds: the durability of mechanical heart valves, and the low thrombogenicity and natural flow characteristics of bioprosthetic heart valves, while overcoming the disadvantages of each of these types of valves.

Many different materials have been used for this application. One of the first materials used was silicone rubber, but it was unable to withstand repeated flexions 
associated with opening and closure (Chetta and Lloyd 1980; Parfeev, Grushetskii et al. 1982). Polytetrafluoroethylene was also used as heart valve material, but it was not able to resist thrombosis and calcification (Imamura and Kaye 1977). In recent times, prosthetic valves made of different forms of polyurethane are being tested (Chetta and Lloyd 1980; Bernacca, Mackay et al. 1995; Bernacca, O'Connor et al. 2002). Polyurethanes have a relatively good biocompatibility, but there have been problems associated with long-term implants (more than 10 years), such as material degradation causing premature failure of devices. Consequently, the development of heart valves made from these materials has been slower than anticipated (Wheatley, Raco et al. 2000). Various studies have shown that failure of bioprosthetic and synthetic valves occurs as a consequence of high tensile and bending stresses acting on the leaflet during opening and closing (Chetta and Lloyd 1980; Bernacca, Mackay et al. 1995; Fann and Miller 1996; Bernacca, O'Connor et al. 2002).

In vitro fatigue testing has shown that a polyetherurethane valve may achieve up to 400 million cycles (Jansen and Reul 1992). This number of cycles represents up to 11 years of fatigue life as number of beats (physiological average of $70 \mathrm{bpm}$ ). This type of valves are still in a testing phase, and not yet commercially available.

\subsection{Polymers in Synthetic Tri-leaflet Heart Valves}

Polymers are synthetic materials composed of long-chains or network of molecules called monomers. Polymers are used as biomaterials because of their ease of manufacturability, processability, low cost, and a wide range of physical and mechanical 
properties. They are classified into thermosets and thermoplastics. Thermosets become permanently hard and rigid when heated, while thermoplastics become soft when heated. Polymers can have crystalline, amorphous, or semicrystalline structures. There are four distinct regions of viscoelastic deformation for polymers. Rigid below the glass transition temperature $\left(\mathrm{T}_{\mathrm{g}}\right)$, leathery near the glass transition temperature, rubbery above the glass transition temperature, and viscous near the melting temperature. Polymers can be characterized by some of their most significant mechanical properties such as tensile strength, Young's modulus, toughness, tensile elongation, and the flexural strength among other mechanical properties. Viscoelastic properties such as stress relaxation and creep can be modeled using mechanical models of material behavior. One of them is the Kelvin model that consists of a spring that is connected in parallel with a dashpot connected to a spring in series. Dynamic mechanical analysis is used to simulate the cyclic loading conditions that a mechanical part might experience in practice. Mechanical properties of polymers are dependant on the rate of application, temperature, and amount of strain. Hooke's law is a good approximation for the relationship between the stress and strain at only small deformations (Shackelford 1996).

As explained before, in the section concerning synthetic tri-leaflet heart valves, in recent years polyurethane has gained the attention of many researchers. However, susceptibility to degradation and mineralization has limited the use of polyurethanes for long-term implants. While surface modification can assist in reducing thrombogenic and calcification reactions (Bernacca and Wheatley 1998), the limiting factor is still longterm biostability of the material itself. Medical uses of polyurethanes have typically been polyester and polyether based formulations. Polyether urethanes degrade by oxidation of 
the ether linkage by cellular substances, typically superoxide and hydrogen ion releases from the granules of leukocytes and macrophages (Zhao, McNally et al. 1993), while the degradation mechanism of polyester urethanes is hydrolysis (Amin, Willie et al. 1993). The effects of degradation are surface cracking and loss of molecular weight, which manifests as a loss of tensile strength of the material. The weak link in these materials is the ester and ether bonds (Pinchuk, Martin et al. 1988). A new class of polycarbonatebased urethanes was designed without ether linkages to eliminate the first site of oxidative attack (Pinchuk, Esquivel et al. 1991). However, it is now clear, that over many years, the next weak links, the urethane and carbonate bonds, slowly degrade by hydrolysis. These degradation modes suggest that the most oxidative stable molecules stand a better chance of survival for ultra long-term use in the body. These molecules must be essentially devoid of heterogeneous linkages in the backbone; they must only contain carbon-carbon linkages; and they must not have the ability to form double bonds between the linkages. Solution, Quatromer (Pinchuk, Khan et al. 1999).

We have chosen to test a composite of Quatromer embedded with polypropylene (PP) fibers. Quatromer is a proprietary polymer that has been found to be less likely to degrade in vivo than polyurethane. It has also been demonstrated to have physical and chemical properties between those of silicone rubber and polyurethane (Pinchuk, Khan et al. 1999). Its ability to withstand boiling concentrated nitric acid suggests that it will perform well in an oxidative and acidic environments such as those found in the body. Two-year testing as microporous stent-grafts demonstrate that that the polymer does not degrade even when implanted next to metal (Pinchuk, Khan et al. 1999). 
Polypropylene fibers have several unique properties that make them especially suited for use as leaflet reinforcement in a synthetic tri-leaflet heart valve. Polypropylene is thermoplastic chemically inert and stable in an alkaline environment. It has a relatively high melting point, and its cost is low. It does not absorb water, due to a hydrophobic surface. Its specific gravity is 0.91 . It is lightest in weight among synthetic fibers, and is one of the strongest. It has little moisture and water absorption. It has a low heat transfer rate, and a melting point of $160^{\circ} \mathrm{C}-170^{\circ} \mathrm{C}$. 


\section{PROBLEM STATEMENT}

Heart valve prostheses have been used for almost half a century in helping individuals with heart valve diseases (Edmunds 2001). Efforts have been directed towards finding the most suitable design and materials for creating the ideal artificial heart valve. Nevertheless, up to date, artificial valves are not as reliable and/or durable as healthy natural ones.

Synthetic tri-leaflet valves are fabricated from biochemically inert synthetic materials, with polyurethane being the typical material of choice. These valves attempt to combine the best of the other two available types of artificial valves: the durability of mechanical valves and the hemodynamic properties of bioprosthetic valves. Although synthetic tri-leaflet valves present natural hemodynamics and have the potential for longterm durability, they have not been successful to date due to long-term material degradation.

It seems reasonable to believe that synthetic tri-leaflet valves might be improved with a suitable material choice. Moreover, it was postulated that a decrease in tears and perforations might result from fiber-reinforced leaflets reducing high stresses on the leaflets. A new material for implant applications, polystyrene-polyisobutylenepolystyrene (Quatromer) is a certain proprietary polymer that has been found to be less likely to degrade in vivo than PU (Pinchuk, Khan et al. 1999). The primary purpose of this study was to explore the possibilities of a new polymer composite to be used as synthetic tri-leaflet heart valve material. This composite was comprised of Quatromer embedded with continuous $10-0$ monofilament $(0.025 \mathrm{~mm}$ diameter $)$ polypropylene fibers 
(Prolene, Ethicon). Polypropylene fibers have several unique properties that make them especially suited for use as leaflet reinforcement in a synthetic tri-leaflet heart valve. Therefore, a Quatromer/polypropylene composite has the potential to become the material of choice for flexible membrane trileaflet heart valves.

In order to determine the feasibility of the Quatromer/polypropylene composite as heart valve material, its static and dynamic mechanical properties were compared with those of an implant-approved polyurethane. 


\section{MATERIALS AND METHODS}

This chapter describes the materials used and procedures followed in the present study. The first section describes the materials that were tested. The second section describes the procedures followed to prepare the specimens of the different materials for each test. Section three describes the procedure followed to carry out the static tests, including the tensile test, Poisson's ratio test, pull-out test, and the creep and relaxation, tests to determine the viscoelastic properties of the different materials. Section four describes the different dynamic tests that were performed. Tests described in this section include the hysteresis test, the tension-tension fatigue test, and the bending fatigue test.

\subsection{Materials}

Materials tested include Quatromer, a polyurethane approved for cardiovascular applications (PU), 10-0 monofilament (25 $\mu \mathrm{m}$ diameter) polypropylene fibers (Prolene, Ethicon) (PP fibers), and a Quatromer/polypropylene (Q/PP) composite.

Quatromer is a triblock composed of a core of polyisobutylene capped on both ends by a harder block of polystyrene, polystyrene-polyisobutylene-polystyrene (Pinchuk, Khan et al. 1999). Quatromer was reinforced with PP fibers. This was the material of choice for our study due to the reasons explained above.

The polyurethane tested in this project was used as a comparative material against Quatromer and the Q/PP composite. Even thought no synthetic tri-leaflet heart valves are manufactured with this polyurethane, it is approved for cardiovascular applications. Up to the date, there are no synthetic tri-leaflet heart valves commercially available. 


\subsection{Specimen Preparation}

Specimens were fabricated of each of the materials prescribed above. The Q/PP composite specimens were prepared as follows. A 20\% Quatromer and $80 \%$ toluene in weight solution was obtained by mixing the Quatromer pellets in toluene with the help of a stirring magnet and a stirring plate. The Quatromer pellets were dried for 24 hours at $70^{\circ} \mathrm{C}$ before mixing. A three-plate assembly was used to mold the solution into rectangular pieces of the desired thickness with the polypropylene threads embedded along the mid-section of the specimens. Caution was taken to insure the PP fibers did not touch each other or cross, and the distance between each other was maintained as constant as possible. However, it cannot be assumed that the fibers were placed totally equidistant from each other (Figure 5).

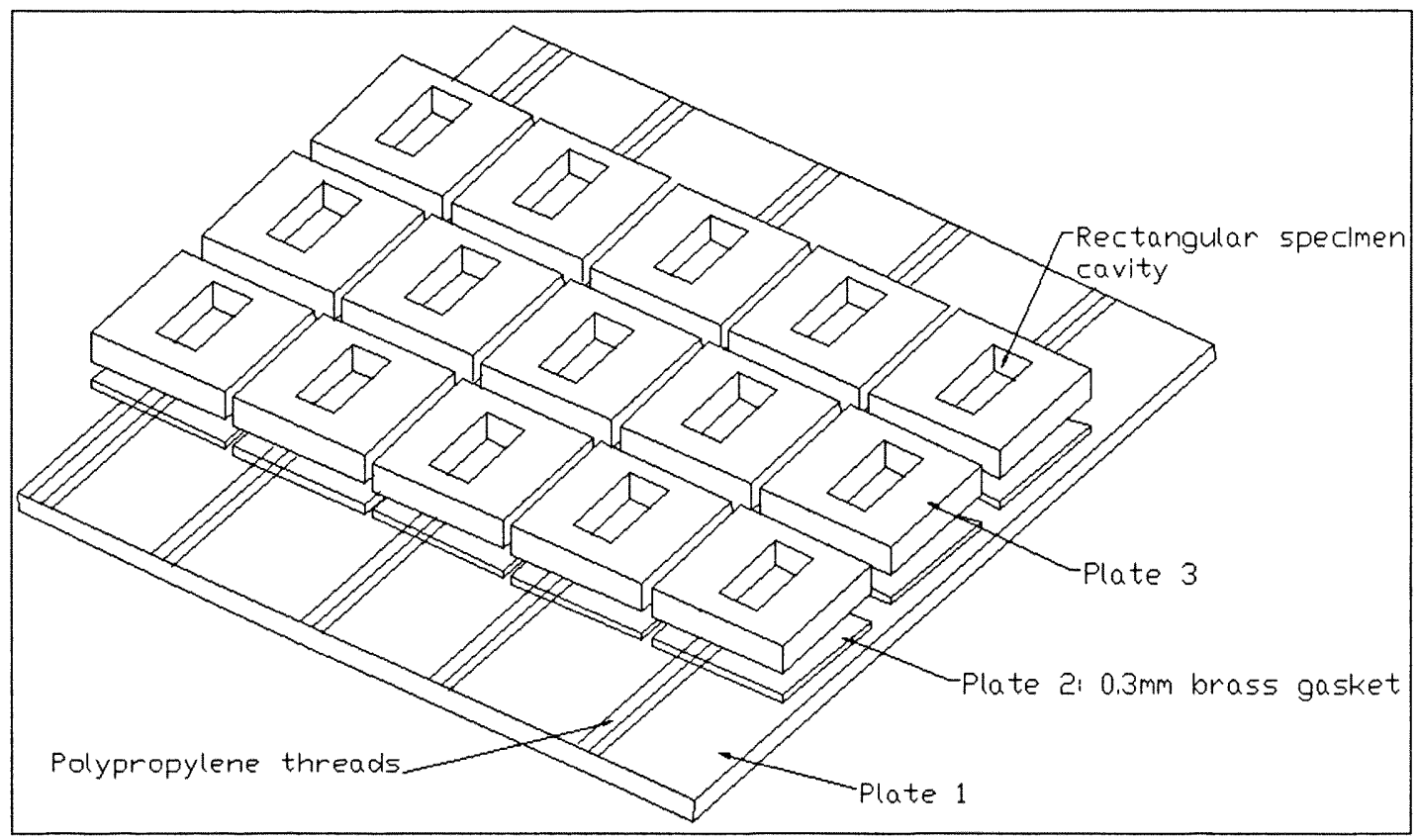

Figure 5. Three-plate assembly used to mold rectangular specimen 
The three-plate assembly was composed of a stainless steel base plate, a set of 15 $0.3 \mathrm{~mm}$ thick brass gaskets (plate 2) with rectangular cavities in the centers, and a set of $151 / 4$ in thick stainless steel plates with rectangular cavities in the centers as depicted in Figure 5. The brass gaskets were placed on top of plate 1 and the desired number of fibers was laid out longitudinally on top of the brass gaskets for each row of specimens. Plate 3 was placed on top after securing the fibers at each end with scotch tape. Six \#2-40 hex socket head screws for each cavity were used to tighten up the assembly. Five $\mathrm{ml}$ of Quatromer solution (for $0.3 \mathrm{~mm}$ thick specimens) were poured into each cavity and the whole plate assembly was placed in an oven at $70^{\circ} \mathrm{C}$ for four hours. All of the plates were thoroughly cleaned with toluene to remove any residue after every usage. The drying process was followed by cutting and inspection processes. A steel cutting die and a hammer were used to cut the rectangular specimens into dog-bone specimens following ASTM standard D 638 - 89, Type M-III (see Figure 6). A caliper was used to measure specimen thickness at three different places on the specimen, each end and the middle. The average specimen thickness obtained was $0.3 \pm 0.01 \mathrm{~mm}$. Specimens that had a thickness variation along its length of more than $10 \%$ were discarded. Figure 7 shows the transverse section of the $\mathrm{Q} / \mathrm{PP}$ composite at the gauge length. This composite has $12 \mathrm{PP}$ fibers embedded in the Quatromer.

Both specimens of Quatromer and PU were prepared following the same procedure as for the Quatromer/PP composite, with the exception that no fibers were embedded. Polypropylene fiber specimens consisted of a single fiber. A compliant and strain-compatible material was used on the end of the specimen to reduce stress concentrations in the gripped area and thereby promote tensile failure in the gage section. 


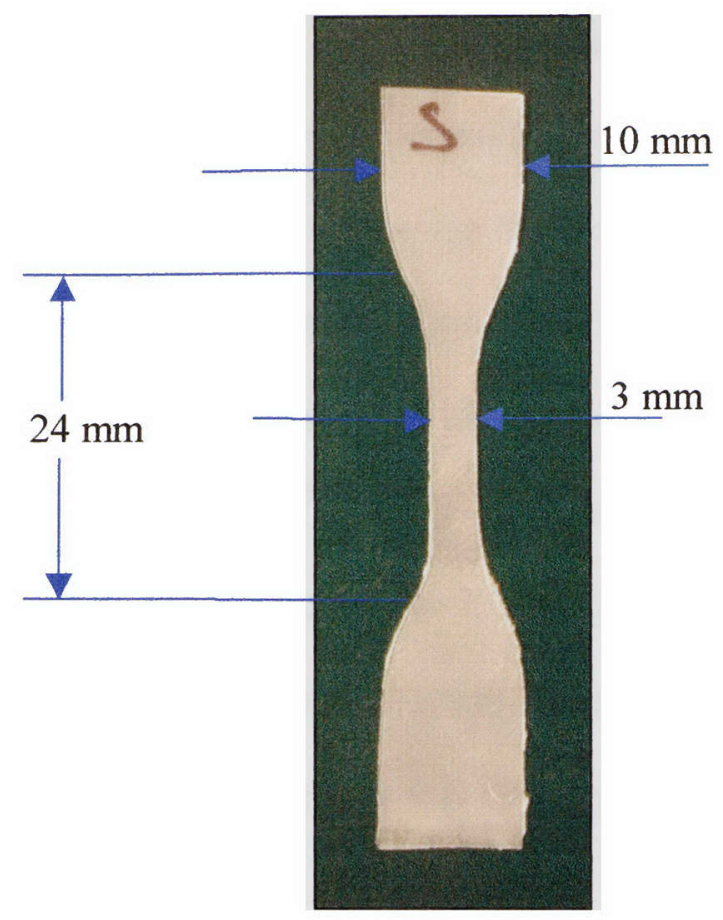

Figure 6. Dimensions of the dog-bone specimen

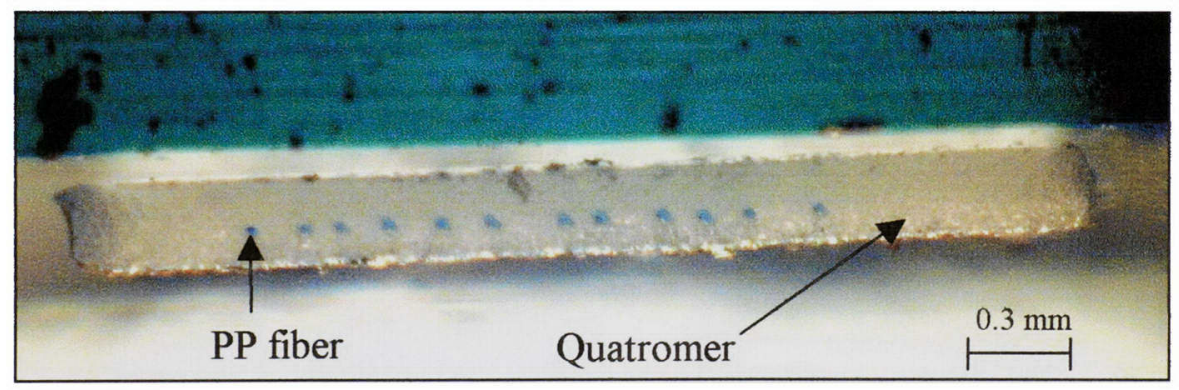

Figure 7. Cross-section view of a $\mathrm{Q}+12$ PP fibers specimen

\subsection{Static Tests}

The static mechanical properties of Quatromer, PU, 10-0 monofilament $(25 \mu \mathrm{m}$ diameter) PP fibers, and a Quatromer/PP composite were determined. The Q/PP composite had 3 or 12 PP fibers embedded in the Quatromer. Four types of static tests 
were performed to each material: a tensile test, to create stress vs. strain data; Poisson's ratio test, to determine the contraction perpendicular to the extension caused by tensile stresses; a pull-out strength test, to determine the bonding forces between the fibers and the matrix; and a test to determine the viscoelastic properties of these materials. The purpose of these tests was to determine the materials static properties. Tensile tests were performed using the Electroforce ${ }^{\mathrm{TM}}$ (ELF) 3200 materials tester (Enduratec Systems Corp., Minnetonka, MN), see Figure 8. The ELF 3200 motor uses direct electromagnetic conversion to apply force. This material tester has a maximum stroke of $12.5 \mathrm{~mm}$, and features a $225 \mathrm{~N}$ motor and has a static to $400 \mathrm{~Hz}$ frequency range.

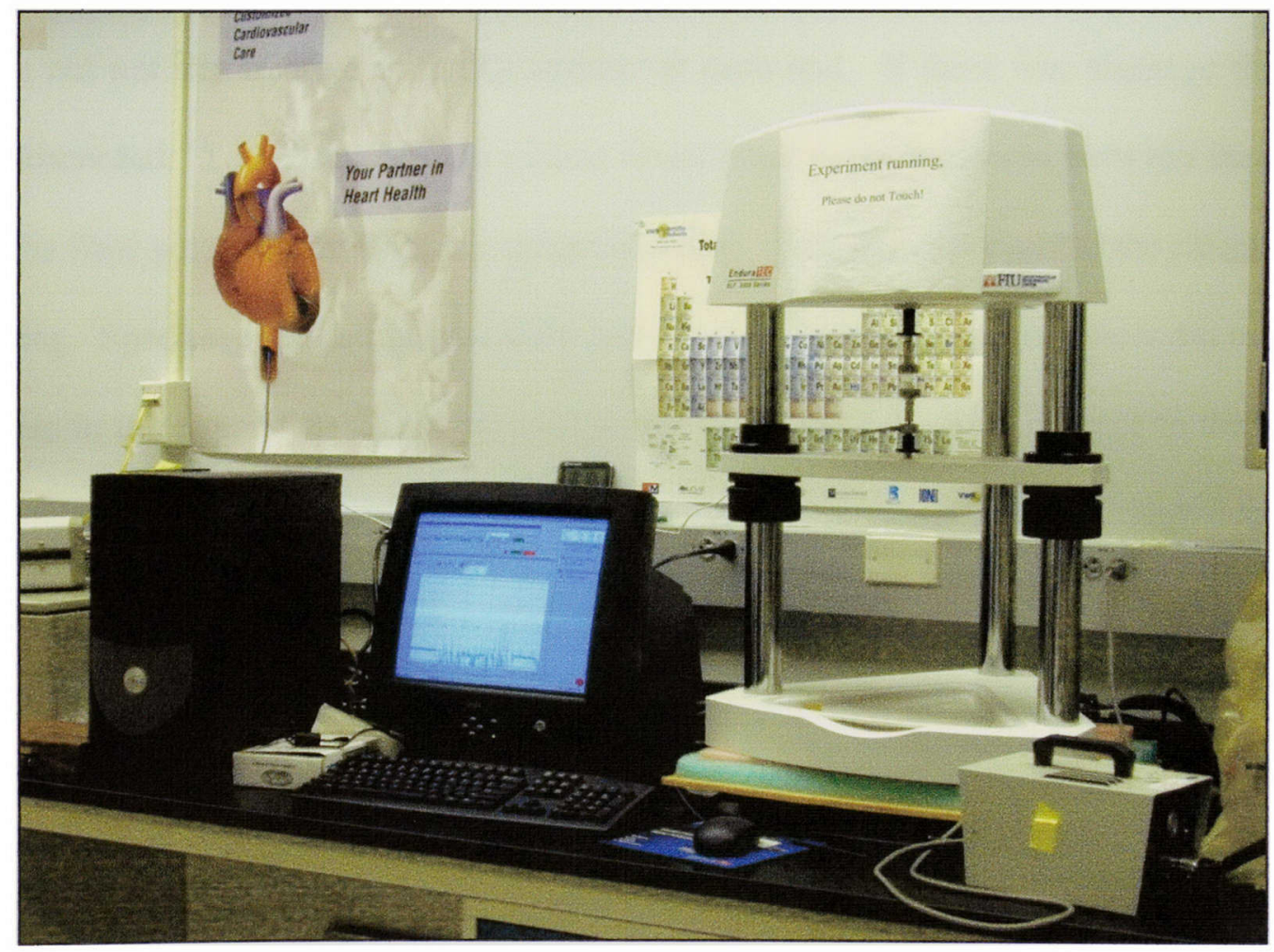

Figure 8. Electroforce ${ }^{\mathrm{TM}}$ (ELF) 3200 materials tester 


\subsubsection{Tensile Test}

Tensile properties of all the materials were performed according to ASTM standards D 638M - 89 (plastics), D 882 - 88 (thin plastic sheets), D 3039 - 89 (composites). Outcome measures include: Young's Modulus (E), ultimate tensile stress (UTS), and ultimate strain (US).

The specimen was held in the testing machine by action grips and pulled at a crosshead speed of $5 \mathrm{~mm} / \mathrm{min}$, which was in accordance with ASTM standards. The specimen was held in a way that slippage relative to the grips was prevented. Grip surfaces are deeply scored to help prevent specimen slippage. Special care was taken for the Q/PP composite. These specimens were checked after each test to make sure that the fibers did not slip relative to the Quatromer at each end. If there was slippage the test was discarded. To test the polypropylene fibers bond paper was used between the grips and the fiber when mounted on the testing machine. This paper prevented the fibers from slipping. Specimens were checked after each test to make sure that the fibers did not slip relative to the paper. Again, in the case where slippage of the fiber relative to the paper occurred, the test was discarded. Tests of any material were not valid if failure occurred at the gripping section. At least five specimens were tested, while taking into consideration that the standard deviation for each of the tests was less than $10 \%$.

\subsubsection{Poisson's Ratio}

Measurement of Poisson's ratio for the Quatromer was carried out in order to determine the contraction perpendicular to the extension caused by tensile stresses. This 
test was performed according to ASTM standard E 132-97. The specimen was held in the same manner as described above for the static tensile tests. Because of the fragility of the samples no extensometers were used. Longitudinal and transverse strain measurements were made as follows.

Four dots $(0.03 \mathrm{~mm}$ in diameter) were marked with India ink at the center of the front side of the specimen in symmetry with the horizontal and vertical axis. These dots formed a square. First a picture of the specimen was taken showing the initial location of the dots as seen in Figure 9a. Then, the specimen was pulled until it reached 15\% strain. Once it reached its maximum elongation (and before relaxation occurred) a second picture was taken showing the new location of the dots, see Figure $9 \mathrm{~b}$. These pictures were taken with the same camera at the same location fixed to the ground. A third picture of a grid, placed on the grips of the tensile machine was taken with the same camera at the same location as before. This picture was used as a reference for the other pictures. Spacing of the lines of the grid is $1 \mathrm{~mm}$.

After the pictures were taken they were analyzed with Photoshop ${ }^{\circledR}$ as follows. The picture of the grid was overlaid on each of the other two pictures. The locations of the points on each picture were measured with reference to the grid. With this information the displacement of the dots were measured with respect to the grid. To reduce measurement errors, a distinguishable location of each dot was used as measurement reference. Poisson's ratio was calculated as follows:

$$
\mu=-\frac{\varepsilon_{t}}{\varepsilon_{l}}
$$




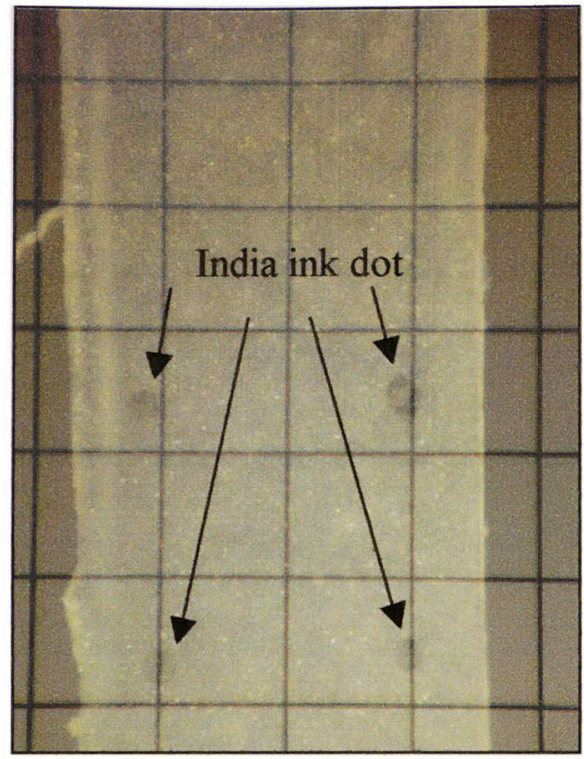

a)

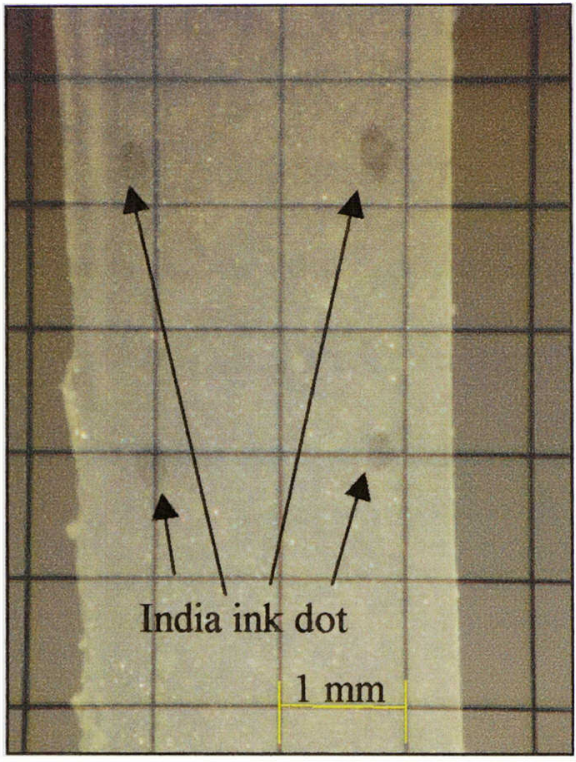

b)

Figure 9. Quatromer specimen mounted on the testing machine showing the location of the four dots at a) $\varepsilon=0 \mathrm{~mm} / \mathrm{mm}$, and b) $\varepsilon=0.15 \mathrm{~mm} / \mathrm{mm}$

where $\mu$ is Poisson's ratio, $\varepsilon_{t}$ is transverse strain, and $\varepsilon_{l}$ is longitudinal strain. Strain $(\varepsilon)$ was defined as:

$$
\varepsilon=\frac{l_{f}-l_{o}}{l_{o}}
$$

where $l_{\mathrm{f}}$ final length, and $\mathrm{l}_{\mathrm{o}}$ is initial length. The same procedure was followed for each specimen. At least five specimens were tested, while taking into consideration that the standard deviation of the results was less than $10 \%$.

\subsubsection{Pull-out Strength Test}

The pull-out strength test was performed in order to determine the properties of the fiber/matrix interface. Specimens used for this test were produced in a similar way as 
those used in the tensile test of the Quatromer/PP composite. These specimens were prepared as follows. First, a Quatromer/PP composite specimen was produced. Secondly, two cuts, (A) and (B), were made with a scalpel blade using the microscope (see Figure 10). This cut was made as close as possible to the fiber without damaging it. Subsequently, the top portion of Quatromer, portion (C) on Figure 10, was carefully removed without damaging the PP fiber. Then the fiber was cut at a distance $\mathrm{L}$ from the beginning of the Quatromer.

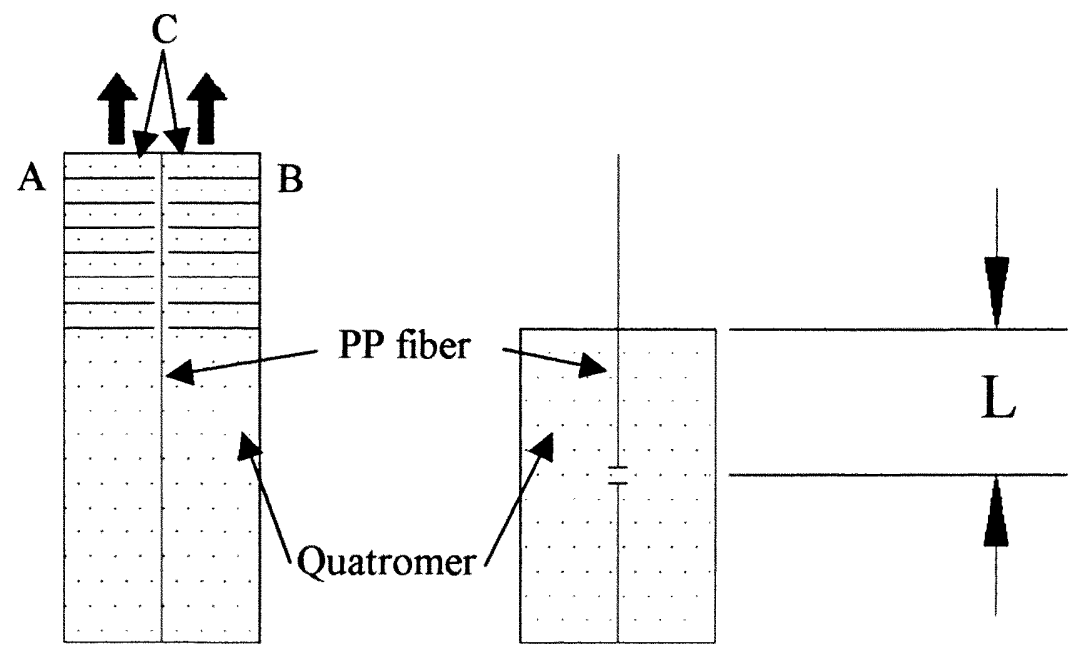

Figure 10. Specimen of Quatromer with 1 PP fiber embedded

Once a portion of the fiber (at least $1 \mathrm{~cm}$ long) was liberated from the Quatromer matrix, it was mounted on the tensile testing machine, see Figure 11. The fiber extended out of the matrix in one end. Close to the other end the fiber was cut using a scalpel blade. The distance of the cut made from the first end determined the length of fiber embedded in the matrix. Caution was taken to cut transversally as less amount of matrix as possible. Specimens with various embedded lengths $(\mathrm{L})$ were tested, enough to create 
an embedded length vs. debonding force $(\mathrm{P})$ curve. Sufficient amount of data points were achieved to fit a line with a coefficient of determination $\left(\mathrm{r}^{2}\right)$ greater than 0.9 . In order to measure the length of the embedded fiber, a picture of the specimen was taken under the microscope, see Figure 12. A $1 \times 1 \mathrm{~mm}$ grid was placed on top of the specimen to determine the correction factor for the measurements. The cut on the specimen caused a portion of the fiber to debond from the matrix. This portion can be noticed in Figure 12 where a white line beside the fiber (blue line) indicates debonding between the fiber and the matrix. Only the portion where this white line is not visible is the portion where the fiber and the matrix are bonded together (L).
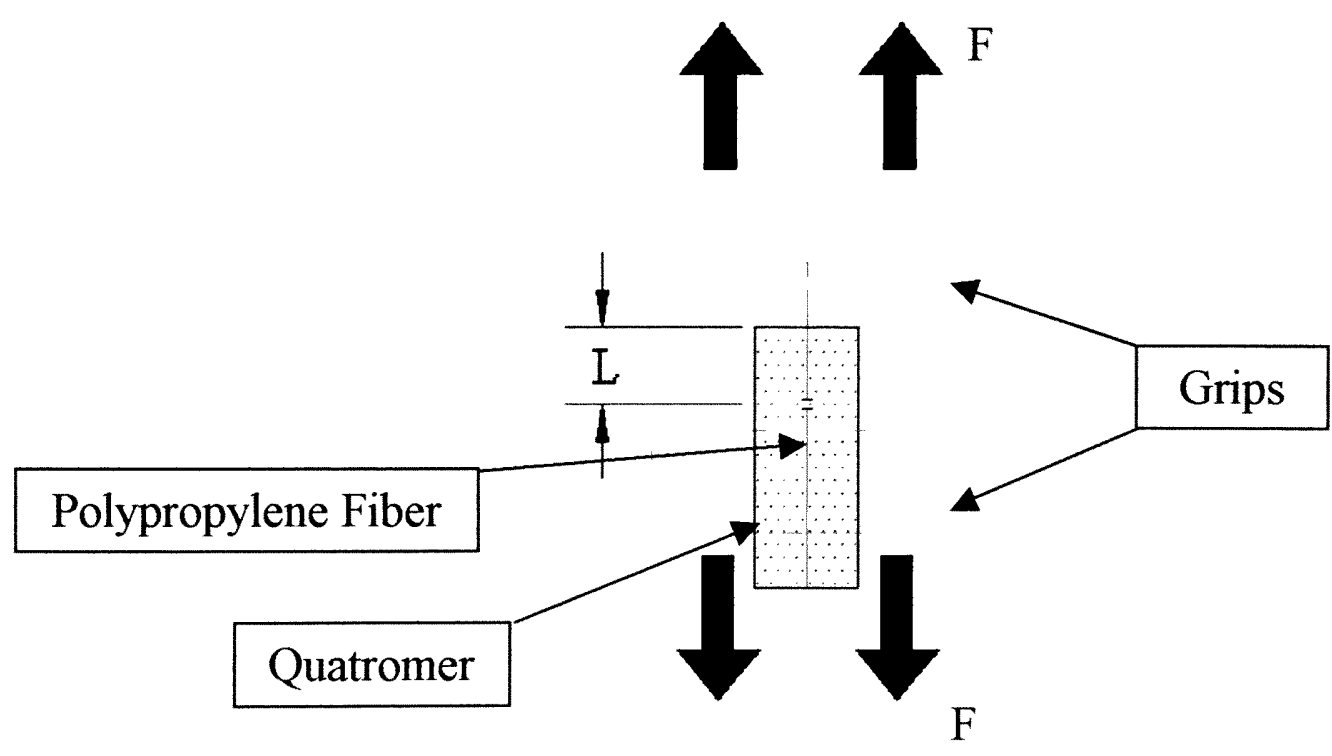

Figure 11. Specimen of Quatromer with 1 PP fiber embedded. The specimen mounted on the testing machine is shown. 


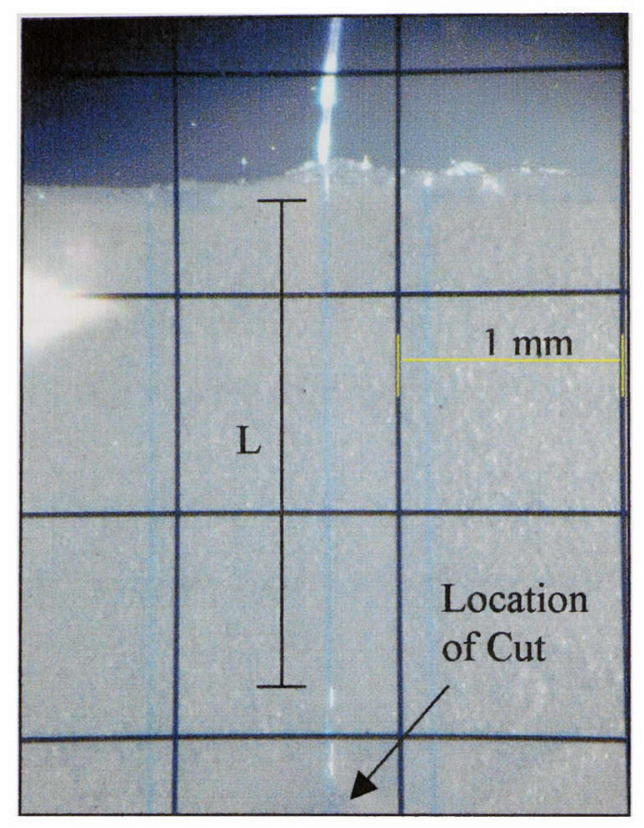

Figure 12. Fiber embedded length.

\subsection{Viscoelastic Properties}

Creep and relaxation curves were created in order to determine the viscoelastic properties between the different materials and compare them. A Kelvin mechanical model was used to describe the viscoelastic behavior of Quatromer, PP fibers, Quatromer/PP composite, and PU. This model consists of a spring connected in parallel to a spring and a dashpot, which are connected in series as seen in Figure 13, where $\mu_{0}$, $\mu_{1}$, and $\eta_{1}$ are constants. $\mathbf{U}$ is the elongation of spring with constant $\mu_{\mathbf{0}}$, which is also the total elongation of the spring and the dashpot $\left(\mu_{1}\right.$ and $\left.\eta_{1}\right) . \mathbf{F}$ is the force applied to the model. For these tests each specimen was held in the same manner as in the tensile test. 


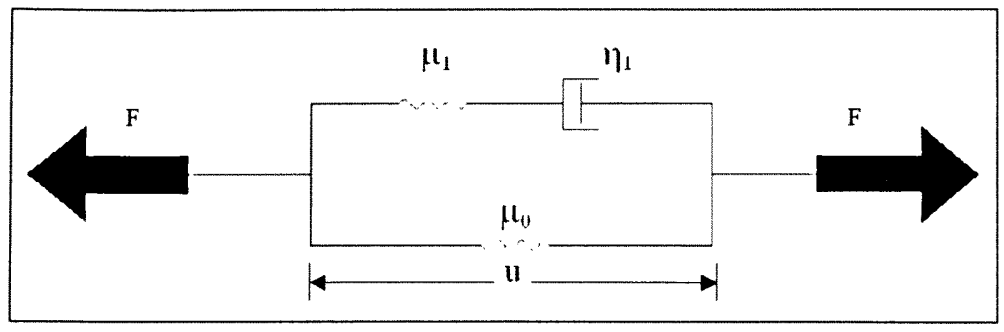

Figure 13. Kelvin Model o Standard linear solid model

The differential equation relating the force and the displacement is given by (Fung 1993)

$$
F+\tau_{\varepsilon} \dot{F}=E_{R}\left(u+\tau_{\sigma} \dot{u}\right),
$$

with initial condition

$$
\tau_{\varepsilon} F(0)=E_{R} \tau_{\sigma} u(0)
$$

where

$$
\tau_{\varepsilon}=\frac{\eta_{1}}{\mu_{1}} \quad, \quad \tau_{\sigma}=\frac{\eta_{1}}{\mu_{0}}\left(1+\frac{\mu_{0}}{\mu_{1}}\right) \quad \mathrm{E}_{\mathrm{R}}=\mu_{0} .
$$

$\tau_{\varepsilon}$ represents the relaxation time for the viscoelastic Kelvin body under constant strain conditions, whereas $\tau_{\sigma}$ represents the retardation time for constant stress (Fung 1993). Equation 4.4 may be simplified to:

$$
\frac{F(0)}{u(0)}=\mu_{1}+\mu_{0}
$$


This equation tells us that the initial response is equal to the sum of the spring constants in the Kelvin model. These represent the elastic response of the material to the stimulus applied.

\subsubsection{Creep}

Creep is the plastic deformation of a material under constant load over a long period of time (Fung 1993). If equation 4.3 is solved for $u(t)$ when $F(t)$ is a unit-step function $1(t)$, the result is the creep function (equation 4.6).

$$
c(t)=\frac{1}{E_{R}}\left[1-\left(1-\frac{\tau_{\varepsilon}}{\tau_{\sigma}}\right) e^{\frac{-t}{\tau_{\sigma}}}\right] l(t),
$$

where the unit-step function $l(t)$ is defined as

$$
l(t)= \begin{cases}1 & \text { when } t>0 \\ \frac{1}{2} & \text { when } t=0 \\ 0 & \text { when } t<0\end{cases}
$$

It represents the elongation produced by a sudden application at $t=0 \mathrm{sec}$. of a constant force of magnitude unity. The creep function is illustrated in Figure 14. The test was load controlled, the input was a force unit-step force applied at $t=0 \mathrm{sec}$. Three specimens of each material were tested. The specimens were stretched to a strain of 0.1 
$\mathrm{mm} / \mathrm{mm}$. Since this was a load controlled test, the amount of force needed to stretch the specimen to $\varepsilon=0.1 \mathrm{~mm} / \mathrm{mm}$ was calculated from the stress vs. strain curves. Once the test was concluded, equation 3.6 was fitted on the data using $\operatorname{SPSS}^{\circledR}$ through the Levenberg-Marquardt nonlinear curve fitting method. The Levenberg-Marquardt method is a single-shot method, which attempts to find the local fit-statistic minimum nearest to the starting point. Its principal advantage is that it uses information about the first and second derivatives of the fit-statistic as a function of the thawed parameter values to guess the location of the fit-statistic minimum. Thus this method works well (and fast) if the statistic surface is well-behaved. Its principal disadvantages are that it will not work as well with pathological statistic surfaces, and there is no guarantee it will find the global fit-statistic minimum (Press, Teukolsky et al. 1988).

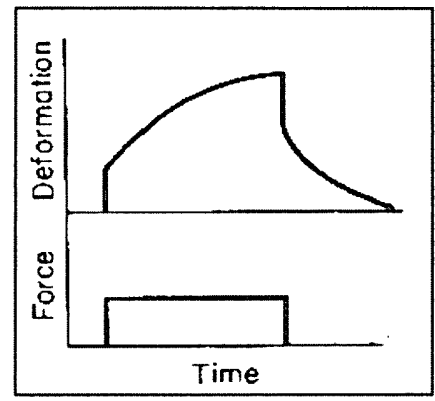

Figure 14. Creep function of a Kelvin Model or Standard linear solid model

\subsubsection{Relaxation}

Relaxation is the response of a body when it is suddenly strained and then the strain is maintained constant afterward. This way, the stresses induced in the body 
decrease with time (Fung 1993). If equation 4.3 is solved for $F(t)$ when $u(t)$ is a unit-step function $1(t)$, the result is the relaxation function (equation 4.8).

$$
k(t)=E_{R}\left[1-\left(1-\frac{\tau_{\sigma}}{\tau_{\varepsilon}}\right) e^{\frac{-t}{\tau_{\varepsilon}}}\right] l(t) .
$$

The relaxation function is illustrated in Figure 15. The test was strain controlled, the input was a strain unit-step function applied at $t=0 \mathrm{sec}$. Three specimens of each material were tested. The specimens were stretched to $\varepsilon=0.1 \mathrm{~mm} / \mathrm{mm}$. Once the test was concluded, equation 3.8 was fitted on the data using SPSS $^{\circledR}$ through the LevenbergMarquardt nonlinear regression estimation method.

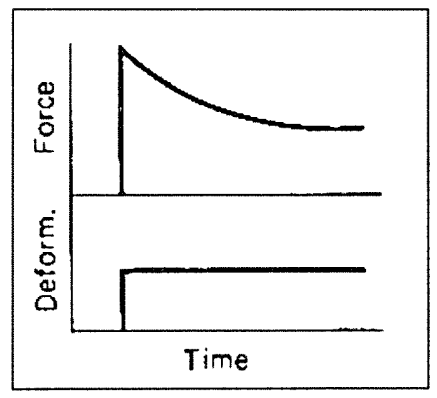

Figure 15. Relaxation function of a Kelvin Model o Standard linear solid model

\subsubsection{Hysteresis}

The phenomenon of hysteresis is present when a body that is subjected to cyclic loading has a different stress-strain relationship in the loading process than in the unloading process. The load frequency for this test was $1.25 \mathrm{~Hz}$. This frequency was based on normal physiological conditions of 75 beats per minute. It was determined 
elsewhere (Liu 2002) that the maximum strain of a Quatromer leaflet under physiological conditions is $0.15 \mathrm{~mm} / \mathrm{mm}$. Based on these results it was decided to strain the specimens this amount.

\subsection{Dynamic Tests}

The fatigue properties of a material represent its response to cyclic loading. It is well recognized that the strength of a material is significantly reduced under cyclic loading (Mallick 1993). A hysteresis curve was created in order to determine the stressstrain relationship in the loading and unloading process of the different materials. Two types of fatigue life analyses were performed: a standard tension-tension fatigue test to create $\mathrm{S} / \mathrm{N}$ (stress vs. number of cycles) data, and a unique bending fatigue test to assess long-term bending effects on material properties. The purpose of these dynamic tests was to provide fatigue properties of the material under tension and bending conditions. They will demonstrate that the Quatromer/PP composite has statistically equal or longer fatigue life than the implant-approved polyurethane. Tension fatigue tests were performed using the Electroforce ${ }^{\mathrm{TM}}$ (ELF) 3200 materials tester (Enduratec Systems Corp., Minnetonka, $\mathrm{MN}$ ). This material tester has a stroke of $12.5 \mathrm{~mm}$, and has a static to $400 \mathrm{~Hz}$ frequency range. Bending tests were performed using the MTS 858 Mini Bionix ${ }^{\circledR}$ system (MTS Systems Corp., Eden Prairie, MN). This system has a stroke of $\pm 50 \mathrm{~mm}$, and it can be operated at frequencies up to $30 \mathrm{~Hz}$. It is capable of testing loads up to $\pm 25 \mathrm{kN}$. 


\subsubsection{Tension-Tension Fatigue Test}

The tension fatigue properties were assessed according to ASTM standard D $3479 \mathrm{M}-96$. The specimens were held in the same manner as in the static tensile tests. This test was load controlled. That is, the specimen was cycled between specified maximum and minimum loads so that constant load amplitude was maintained. Load range was defined as $\pm 10 \%$ the mean load. Load amounts used for each specimen were progressively lowered from the static value until a sufficient amount of data points existed to create a $\mathrm{S} / \mathrm{N}$ curve.

The load frequency was $100 \mathrm{~Hz}$. At this frequency, heating of the specimen may occur. Therefore, the temperature of the specimens was monitored through thermal infrared imaging techniques to insure it did not exceed $100{ }^{\circ} \mathrm{C}$ (glass transition temperature, $\mathrm{T}_{\mathrm{g}}=165^{\circ} \mathrm{C}$ ). Specimens were tested in air. Cycling was performed for each polymer until failure. Since there could be significant fatigue damage without actual fracture, failure was defined as a strain of $0.5 \mathrm{~mm} / \mathrm{mm}$. This failure criterion is based on the knowledge that normal maximum local strains in natural heart valves vary between $10-20 \%$. Above $20 \%$ it is not likely to be functional. We decided to test beyond this limit to fully characterize the material in extreme conditions. Also, this was the maximum strain that could be reached with the tensile testing machine and the dimensions of the specimens used. After 350 million cycles without failure the test was stopped and the material was considered with infinite life for that stress. At this point the stress applied to the material appeared to be below its endurance limit. This number of cycles represents up to 10 years of fatigue life as number of beats (physiological average of $70 \mathrm{bpm}$ ). 


\subsubsection{Bending Fatigue Test}

The investigator is not aware of an ASTM or ISO standard test for bending fatigue of an elastomeric material. Nevertheless, it is well known that valve leaflets are subjected to pure bending in normal flow conditions (Bernacca, Mackay et al. 1995). The effects of cyclic bending on the test materials were assessed as follows. The specimens were loaded as in the Standard Tension Fatigue configuration, but in buckling rather than in tension mode. In this case the test was deflection controlled, rather than load controlled as in the tension fatigue tests.

By image analysis it was determined that the deflection of the specimen produced a major curvature of $0.296 \mathrm{~mm}^{-1}$ as shown in Figure 16. This is in agreement with curvatures that have been measured for prosthetic valve leaflets in vitro (Lyengar, Sugimoto et al. 2001). A polymer with zero residual strain after cyclic loading is normally thought to be durable and have good fatigue tolerance properties. Therefore, a stiffness reduction analysis was used. Five groups of specimens were tested. Each group was formed by 5 specimens of Quatromer and 5 specimens of Q +12 PP fibers. These groups were cycled at $12 \mathrm{~Hz}$ for 7, 16, 57.5, 79, and 89 million cycles (see Table 1). After cycling, each specimen was tested (within a one hour lapse) according to the Static Test Protocols listed above. A change in the material properties indicated impairment due to fatigue. 


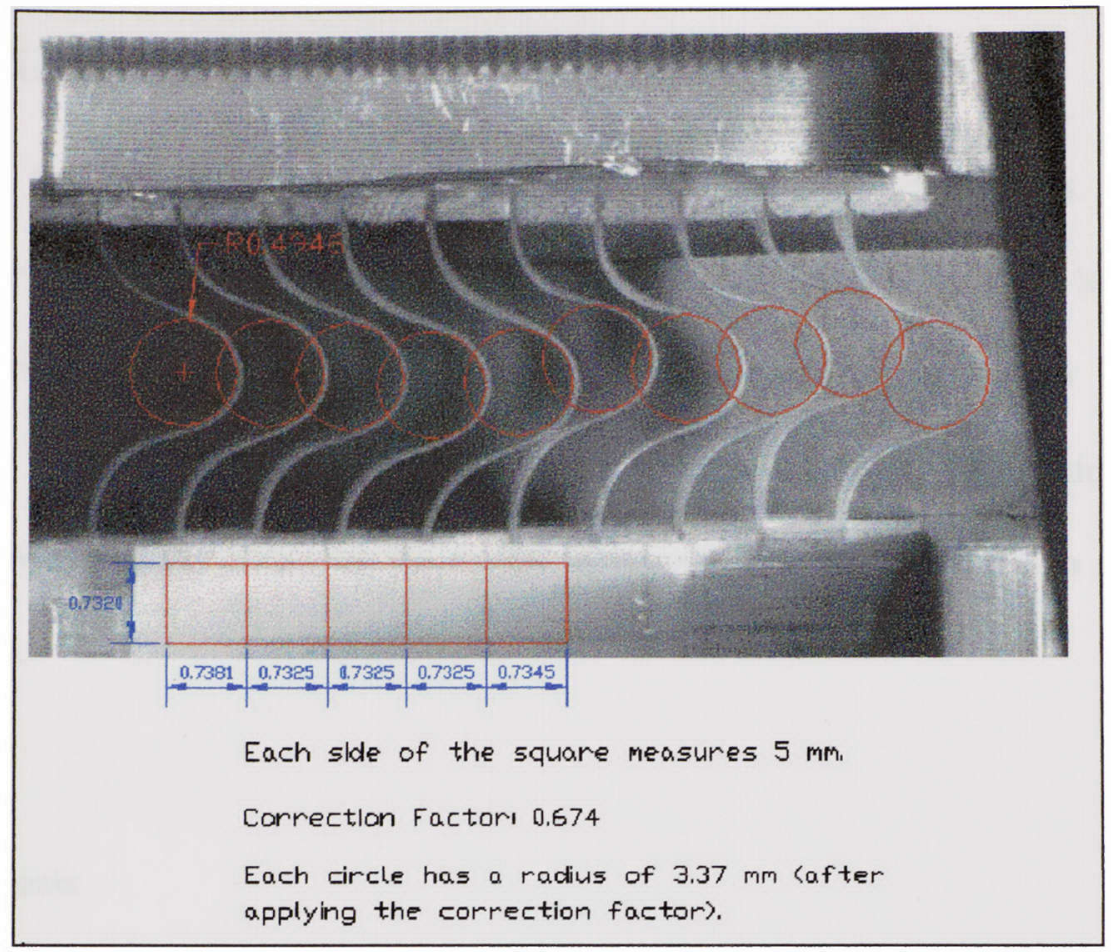

Figure 16. Measurement of the curvatures produced in the bending fatigue test.

Table 1. Number of years equivalent to bending cycles

\begin{tabular}{|c|c|c|}
\hline Group & $\begin{array}{c}\text { Number of Bending Cycles } \\
\text { (million) }\end{array}$ & $\begin{array}{c}\text { Equivalent Time at } \\
\text { Physiological Rate }(\mathbf{7 0} \mathbf{b p m})\end{array}$ \\
\hline 1 & 0 & 0 \\
2 & 7 & 65 days \\
3 & 16 & 148 days \\
4 & 57.5 & 1.5 years \\
5 & 79 & 2 years \\
6 & 89 & 2.3 years \\
\hline
\end{tabular}




\section{RESULTS}

The first section presents the results of the static mechanical tests. Within this section, the results of the tensile test, pull-out test, poison's ratio test, and the determination of the viscoelastic properties for each of the materials studied, are presented. In the second section the results of the dynamic mechanical tests are presented, which include the results of the hysteresis, and tension-tension and bending fatigue tests.

\subsection{Static Tests}

\subsubsection{Tensile Tests}

Tensile tests were carried out in order to create a stress vs. strain curve for each material. Figure 17 shows the stress vs. strain curves for Quatromer, Q + 3 PP fibers, PU, and Q + 12 PP Fibers. Figure 18 shows the stress vs. strain curve for 1 PP fiber.

As indicated by Figure 17, PU had a significantly higher strength than Quatromer. Its Young's modulus was almost five times greater than for Quatromer (see Table 2 for numerical results of the different materials). Young's modulus was measured in the initial 10\% in strain. In Figure 17, Quatromer and PU are shown to have an elongation up to $0.5 \mathrm{~mm} / \mathrm{mm}$. These two materials did not fail at this strain; the tensile machine was not capable to elongate them any further (because it reached its maximum stroke) so the test was stopped at this strain. PU showed a higher strength than Quatromer, but once Quatromer was embedded with PP fibers its strength increased even surpassing the PU. 


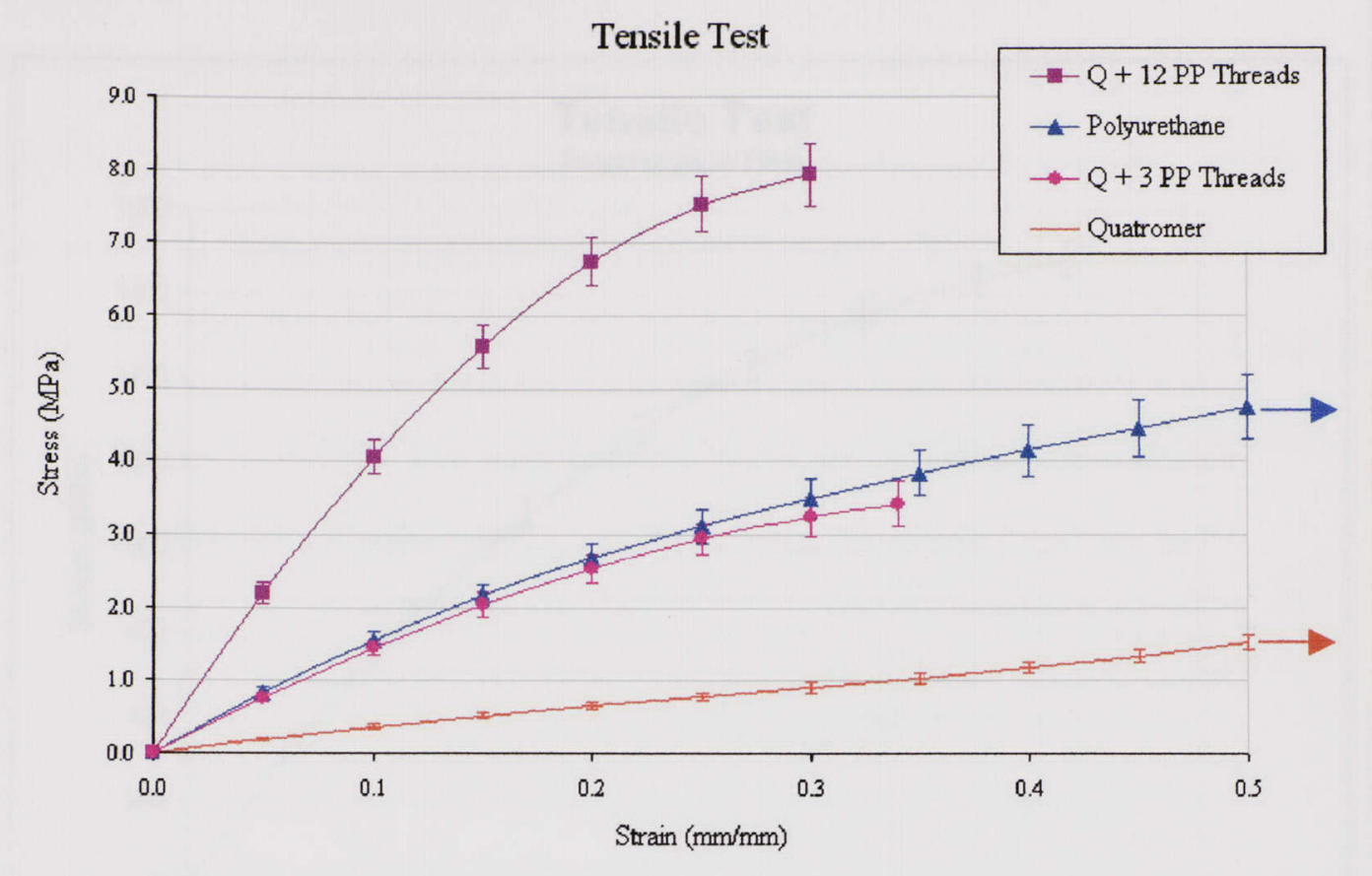

Figure 17. Stress vs. Strain graph of the different materials The error bars represent one standard deviation

For Q + 3 PP fibers its strength was in the range of the PU. This composite failed at a strain of $0.35 \mathrm{~mm} / \mathrm{mm}$, when the PP fibers broke. Q + 12 PP fibers showed a much higher strength than PU, its Young's modulus was more than 2 times greater than for PU. $\mathrm{Q}+12 \mathrm{PP}$ fibers strain at failure was $0.3 \mathrm{~mm} / \mathrm{mm}$. 


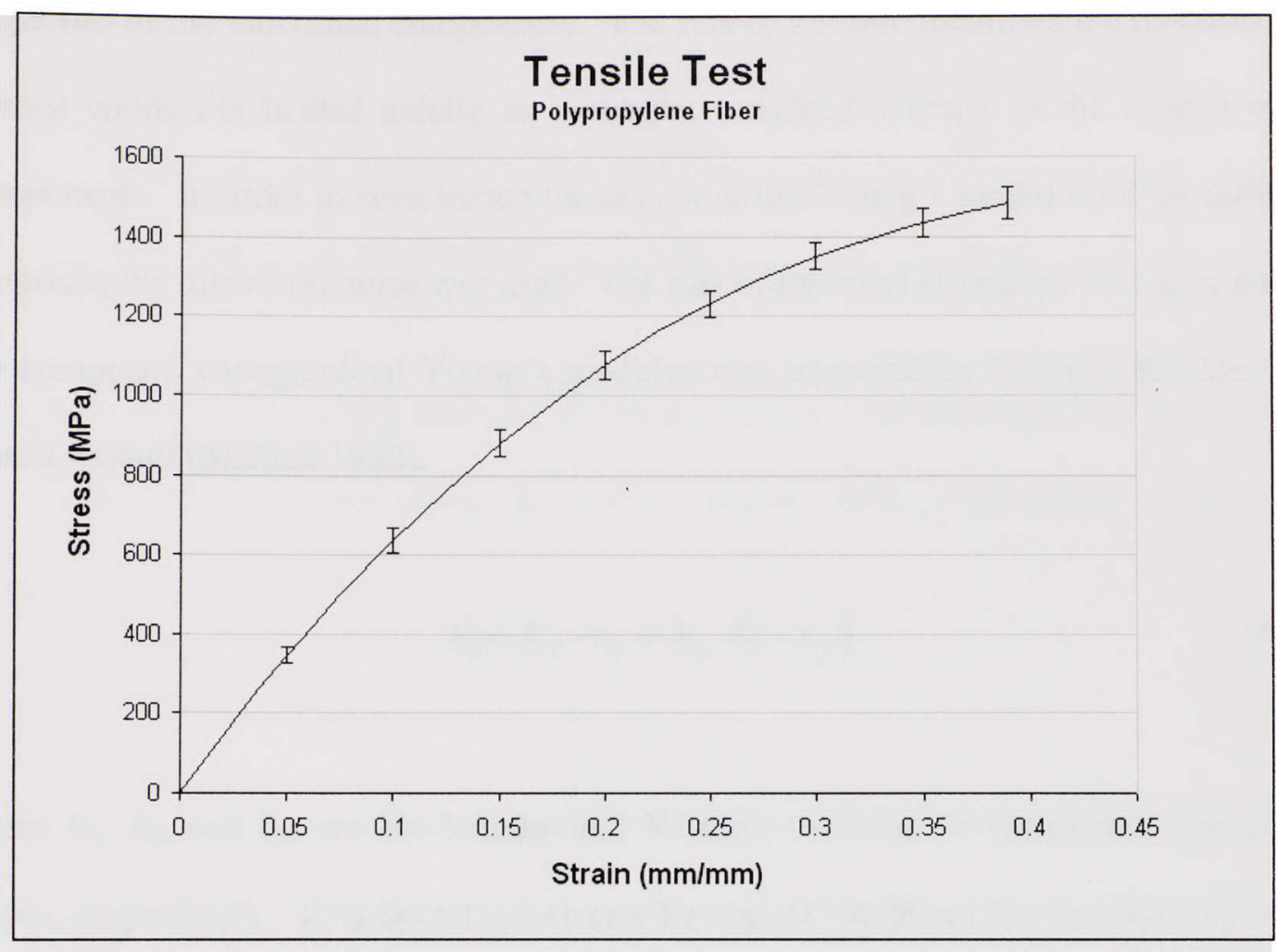

Figure 18. Stress vs. Strain graph of polypropylene fibers

The error bars represent one standard deviation.

Table 2. Young's modulus (E), Ultimate Strain (US), Ultimate Tensile Stress (UTS), and Number of specimens tested $(\mathrm{N})$ of each material

\begin{tabular}{|l|c|c|c|c|}
\hline \multicolumn{1}{|c|}{ Material } & $\begin{array}{c}\mathbf{E} \\
(\mathbf{M P a})\end{array}$ & $\begin{array}{c}\text { US } \\
(\mathbf{m m} / \mathbf{m m})\end{array}$ & $\begin{array}{c}\text { UTS } \\
(\mathbf{M P a})\end{array}$ & $\mathbf{N}$ \\
\hline Quatromer (Q) & $3.88 \pm 0.40$ & $>0.5$ & $1.43 \pm 0.15$ & 22 \\
\hline PP Fiber & $6,633.65 \pm 492$ & $0.43 \pm 0.05$ & $1,543.54 \pm 124$ & 14 \\
\hline Q + 3 PP Fibers & $14.93 \pm 2.67$ & $0.34 \pm 0.05$ & $3.40 \pm 0.55$ & 8 \\
\hline Polyurethane & $18.53 \pm 1.23$ & $>0.5$ & $5.44 \pm 0.41$ & 13 \\
\hline Q+12 Fibers & $45.44 \pm 2.85$ & $0.30 \pm 0.03$ & $7.92 \pm 0.87$ & 10 \\
\hline
\end{tabular}


The properties of the composite, in some way, represent an average of the properties of the individual components. The rule of mixture identifies the modulus of a fibrous composite loaded axially as a simple, weighted average of the moduli of its components. In order to corroborate the results of the Young's modulus of the different materials, the rule of mixtures was used. The rule of mixtures (Equation 5.1) showed that the composite's longitudinal Young's modulus was intermediate between the fiber and matrix moduli (Mallick 1988).

$$
E_{c}=E_{f} \cdot v_{f}+E_{m} \cdot\left(1-v_{f}\right)
$$

where $E_{c}, E_{f}$, and $E_{m}$ are the longitudinal Young's modulus of composite, fibers, and matrix, respectively. $V_{\mathrm{f}}$ is the ratio between the area of the fiber $\left(\mathrm{A}_{\mathrm{f}}\right)$, and the area of the composite $\left(A_{c}\right)$ (see Equation 5.2). Table 3 shows the values of $A_{f}, A_{c}, E_{f}$, and $E_{m}$, which were used in equation 5.1. These values represent the previously measured mean values. Table 4 shows the results of Young's modulus calculated with the rule of mixture and the experimental results.

$$
v_{f}=\frac{A_{f}}{A_{c}}
$$

Table 3. Values for $A_{f}, A_{c}, E_{f}$, and $E_{m}$

\begin{tabular}{|c|c|c|c|c|}
\hline $\begin{array}{c}\mathbf{A}_{\mathbf{f}}(\mathbf{e a c h}) \\
\left(\mathbf{m}^{\mathbf{2}}\right)\end{array}$ & $\begin{array}{c}\mathbf{A}_{\mathbf{c}}(\mathbf{Q}+\mathbf{3} \text { PP Fibers }) \\
\left(\mathbf{m}^{\mathbf{2}}\right)\end{array}$ & $\begin{array}{c}\mathbf{A c}(\mathbf{Q}+\mathbf{1 2} \text { PP Fibers }) \\
\left(\mathbf{m}^{\mathbf{2}}\right)\end{array}$ & $\begin{array}{c}\mathbf{E}_{\mathbf{f}} \\
(\mathbf{M P a})\end{array}$ & $\begin{array}{c}\mathbf{E}_{\mathbf{m}} \\
(\mathbf{M P a})\end{array}$ \\
\hline $4.91 \mathrm{E}-10$ & $9.00 \mathrm{E}-07$ & $8.40 \mathrm{E}-07$ & 6,634 & 3.88 \\
\hline
\end{tabular}


Table 4. Volume fraction of the fibers $\left(\mathrm{V}_{\mathrm{f}}\right)$, and theoretical (rule of mixture) and experimental values of the composite's Young's modulus $\left(\mathrm{E}_{\mathrm{c}}\right)$.

\begin{tabular}{|c|c|c|c|}
\hline \# of Fibers & $\begin{array}{c}\mathbf{V}_{\mathbf{f}} \\
\left(\mathrm{m}^{2} / \mathrm{m}^{2}\right)\end{array}$ & $\begin{array}{c}\text { Rule of Mixture } \\
(\mathrm{MPa})\end{array}$ & $\begin{array}{c}\text { Experimental Results } \\
(\mathrm{MPa})\end{array}$ \\
\hline $\mathbf{3}$ & $1.64 \mathrm{E}-03$ & 14.73 & 14.93 \\
$\mathbf{1 2}$ & $6.54 \mathrm{E}-03$ & 50.5 & 45.44 \\
\hline
\end{tabular}

The load contribution of the fibers to the composite was calculated by the fraction of the total composite load carried by the axially loaded fibers (Shackelford 1996), (see Equation 5.3).

$$
\frac{P_{f}}{P_{c}}=\frac{E_{f}}{E_{c}} \cdot V_{f}
$$

where $P_{f}$ and $P_{c}$ are the fiber and composite loads, respectively. For $Q+3 P P$ fibers, $P_{f} /$ $\mathrm{P}_{\mathrm{c}}=0.73$; that is almost $3 / 4$ of the entire uniaxial load was carried by $0.16 \mathrm{vol} \%$ of high modulus fibers, while for the $\mathrm{Q}+12 \mathrm{PP}$ fibers composite, $\mathrm{P}_{\mathrm{f}} / \mathrm{P}_{\mathrm{c}}=0.95$; that is nearly the entire uniaxial load was carried by 0.65 vol \% of high modulus fibers.

\subsubsection{Poisson's Ratio}

Poisson's ratio of Quatromer was performed in order to determine the contraction perpendicular to the extension caused by tensile stresses. This test was performed following the methods described in Section 4.2.2. Results are presented on Table 5. The negative sign on the strain indicates the distance between points is decreased, while a 
positive sign indicates the distance between the points is increased. Five specimens were tested.

Table 5. Strain $(\varepsilon)$ and Poisson's Ratio measurements of Quatromer ( $\varepsilon$ units are in $\mathrm{mm} / \mathrm{mm}$ )

\begin{tabular}{|c|cc|c|}
\hline Sample & \multicolumn{2}{|c|}{$\boldsymbol{\varepsilon}$} & Poisson's Ratio \\
\hline $\mathbf{1}$ & Horizontal $(\mathbf{x})$ & Vertical $(\mathbf{y})$ & \\
$\mathbf{2}$ & -0.0614 & 0.1480 & 0.415 \\
$\mathbf{3}$ & -0.0560 & 0.1426 & 0.393 \\
$\mathbf{4}$ & -0.0654 & 0.1481 & 0.409 \\
$\mathbf{5}$ & -0.0569 & 0.1580 & 0.414 \\
\hline Mean & -0.0601 & 0.1433 & 0.397 \\
Std. Dev. & 0.00378 & 0.1480 & $\mathbf{0 . 4 1}$ \\
\hline
\end{tabular}

\subsubsection{Pullout Strength Test}

The pull-out strength test was performed in order to determine the properties of the fiber/matrix interface. This test was performed following the methods described in section 4.3.3. Figure 19 shows a typical force vs. displacement plot for a single fiber of the pull-out test. Pull-out tests for all fibers displayed a similar pattern. The following features are worth noting. First, a linear rise in force (A) is followed by an instantaneous drop in force. In this initial stage, between 0 and $\mathrm{A}$, the fiber is physically and chemically locked. Subsequently, a second rise occurs up to a second peak (B). At this point, between $\mathrm{A}$ and $\mathrm{B}$, the fiber becomes physically locked (chemical bonding no longer exists) since the relaxation of the strain energy in the fiber has caused resumption of the original diameter (Poisson's ratio effect) and contact between the fiber and matrix internal surface. The lateral compressive forces resulting from resin shrinkage dictate this 
'static' frictional force; it is, therefore, related to fiber length. Finally, a second sudden drop occurs followed by a linear fall of force.

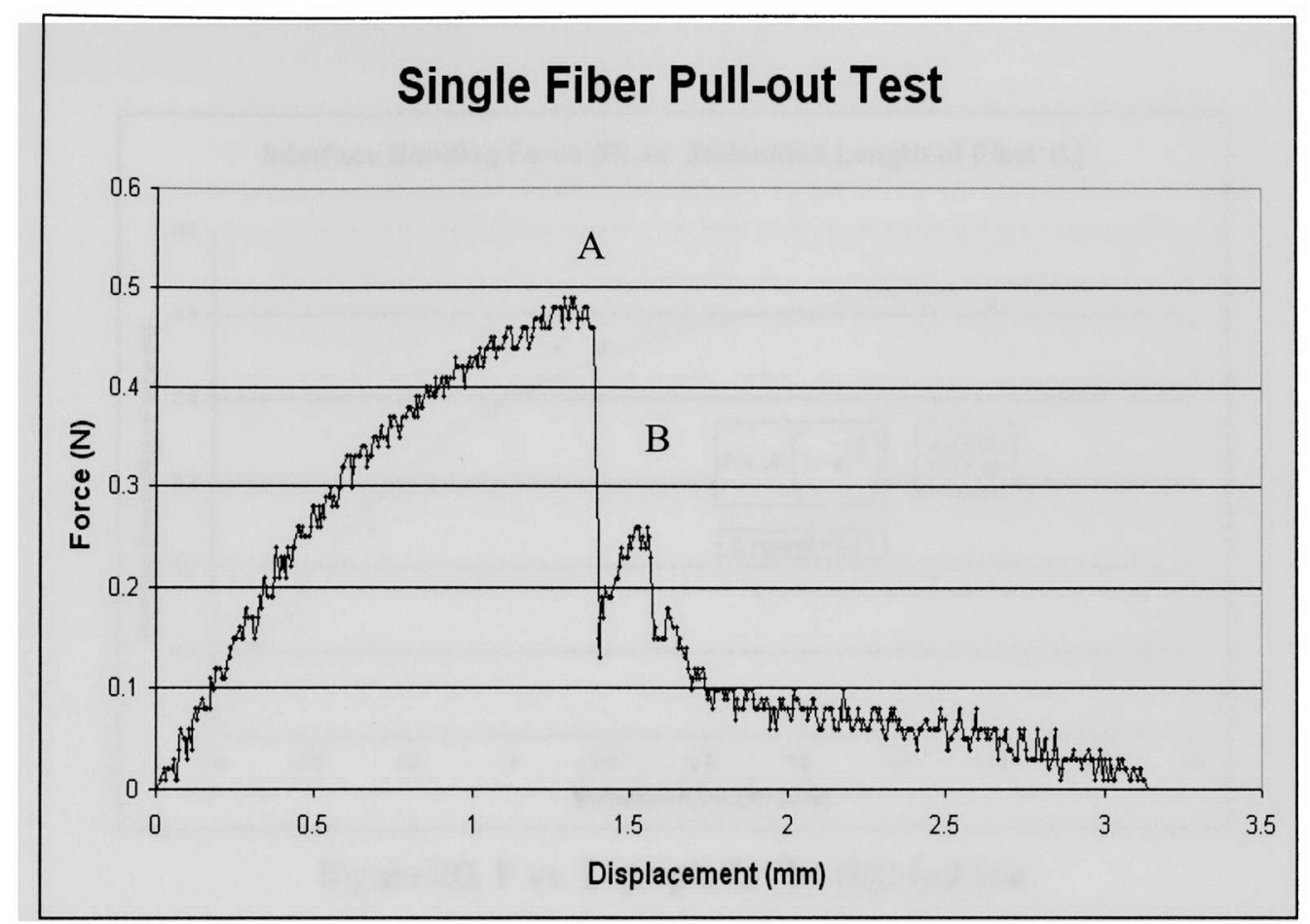

Figure 19. Typical plot of a single fiber pull-out

Figure 20 displays the interface bonding force (P) vs. embedded length of fiber (L) of the pull-out test. This graph shows a rising portion of the plot followed by a plateau. The force never exceeds $0.5 \mathrm{~N}$, which is the tensile strength of the PP fiber. The results were fitted to an exponential function of the form:

$$
P=A \cdot\left(1-e^{-\frac{L}{B}}\right)
$$


where, A and B are constants. These constants represent the plateau and the time constant, respectively.

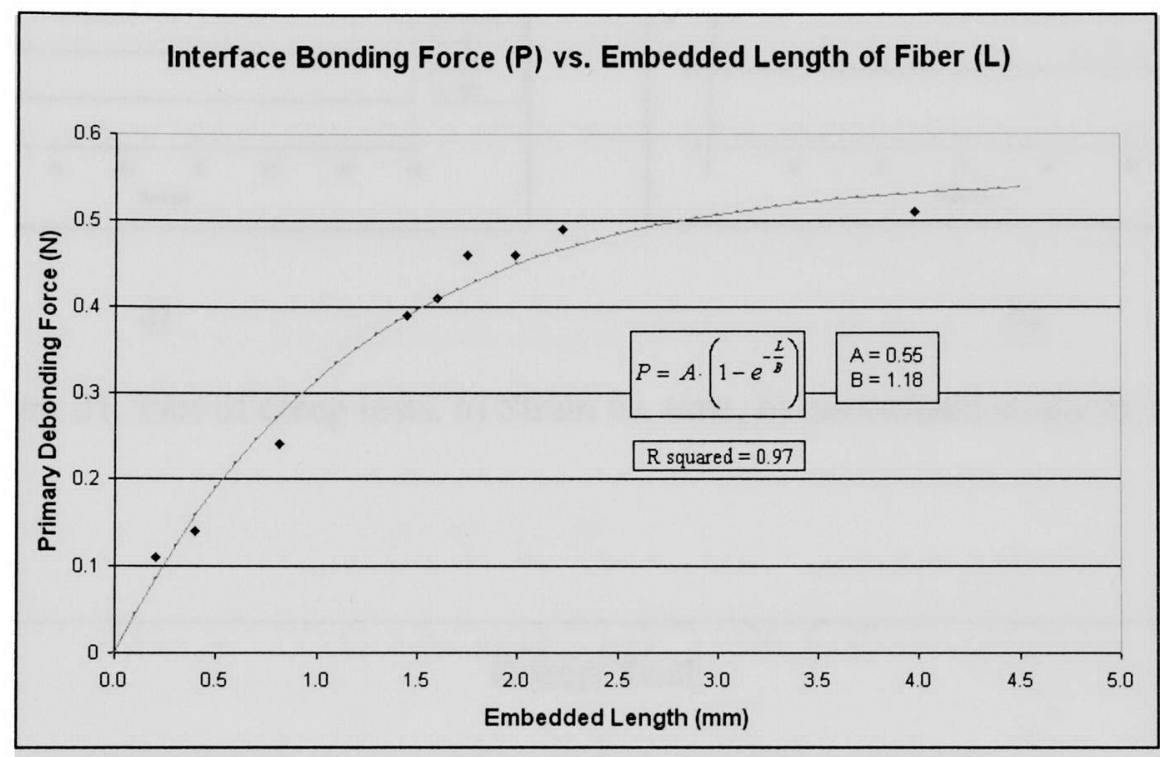

Figure 20. P vs. L graph for the pull-out test

\subsection{Viscoelastic Properties}

\subsubsection{Creep}

Creep tests were performed in order to characterize the plastic deformation of each of the materials over a long period of time. Figure 21 shows the results of the creep tests. Figure $21 \mathrm{a}$, shows the strain vs. time, while Figure $21 \mathrm{~b}$ shows normalized strain vs. time of the creep curves of the different materials. Three specimens were tested for each material. Figure 22 shows the initial 10 seconds of each normalized curve. In this figure, the initial response of each material is shown in more detail. Figure 22 shows that Q and 


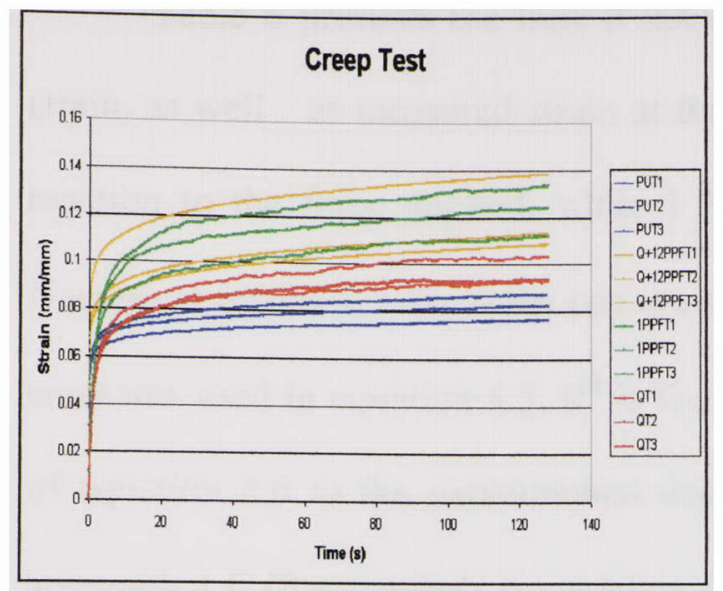

a)

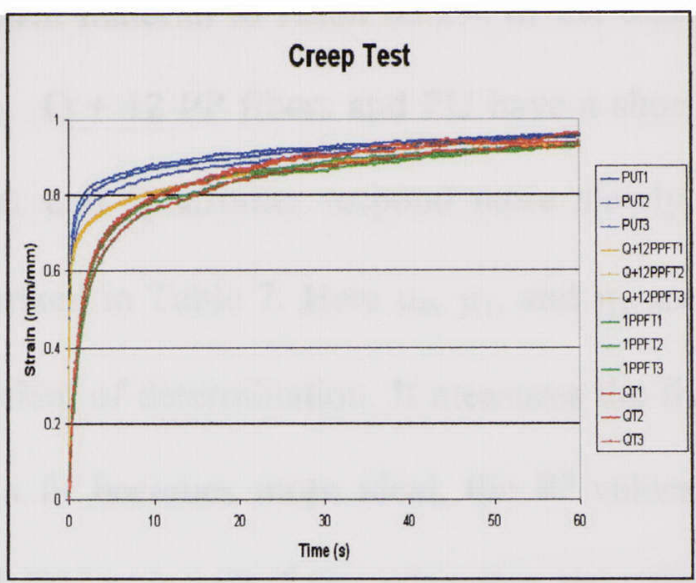

b)

Figure 21. Plot of creep tests. a) Strain vs. time, b) normalized strain vs. time

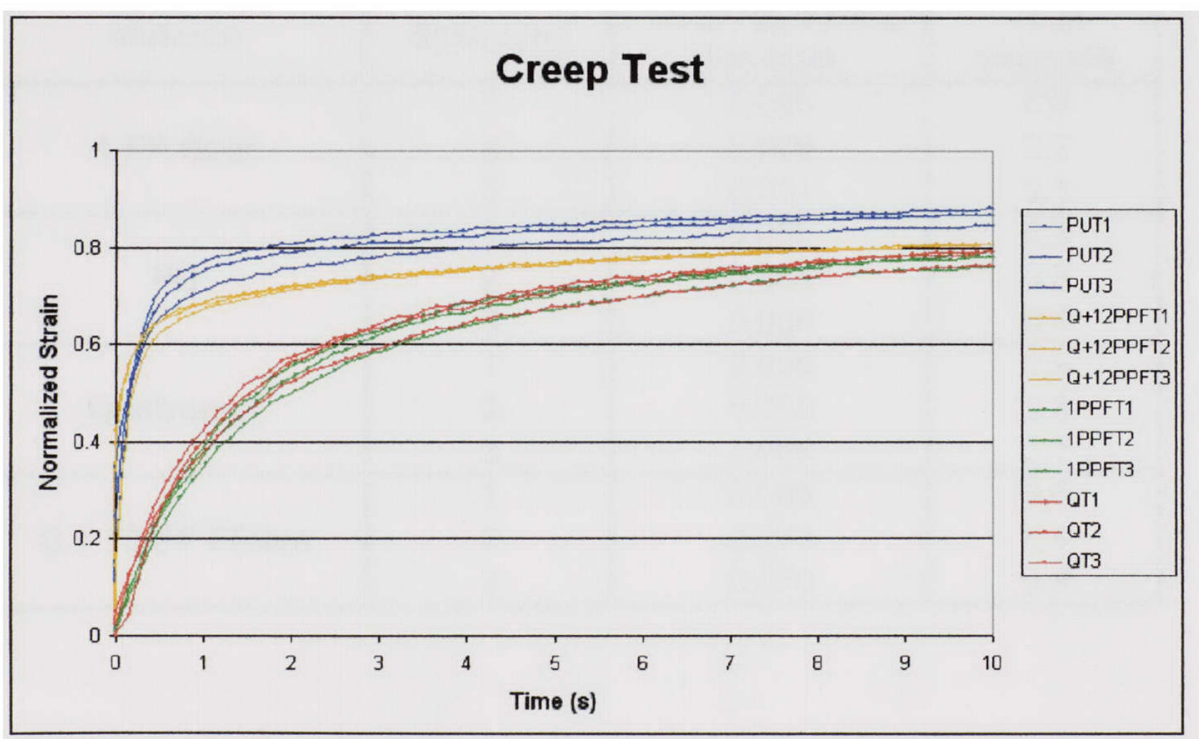

Figure 22. Normalized stress vs. time creep test results (initial 10 seconds)

Table 6 presents the time it takes for each material to reach $63.2 \%$ of the total strain, as well as measured strain at this time. Q + 12 PP fibers and PU have a short 
Table 6 presents the time it takes for each material to reach $63.2 \%$ of the total strain, as well as measured strain at this time. $\mathrm{Q}+12 \mathrm{PP}$ fibers and PU have a short reaction to the force applied, while $1 \mathrm{PP}$ fiber and Quatromer respond more slowly. Numerical results for the creep test are summarized in Table 7. Here $\mu_{0}, \mu_{1}$, and $\eta_{1}$ are constants used in equation $4.5 . \mathrm{R}^{2}$ is the coefficient of determination. It measures the fit of equation 4.6 to the experimental data. As a fit becomes more ideal, the $\mathrm{R}^{2}$ values approach 1.0 ( 0 represents a complete lack of fit). $\tau_{\varepsilon}$ is called the relaxation time for constant strain, whereas $\tau_{\sigma}$ is called the relaxation time for constant stress.

Table 6. Numerical summary of the creep test

\begin{tabular}{|c|c|c|c|}
\hline Material & Specimen & $\begin{array}{c}\text { Strain @ 63.2\% } \\
\mathrm{mm} / \mathbf{m m}\end{array}$ & $\begin{array}{c}\text { Time } \\
\text { seconds }\end{array}$ \\
\hline \multirow{2}{*}{ 1 PP fiber } & 1 & 0.085 & 4.0 \\
& 2 & 0.079 & 3.2 \\
& 3 & 0.071 & 3.1 \\
\hline \multirow{2}{*}{ PU } & 1 & 0.050 & 0.3 \\
& 2 & 0.048 & 0.3 \\
Quatromer & 3 & 0.056 & 0.4 \\
\hline \multirow{2}{*}{ Q + 12 PP Fibers } & 2 & 0.059 & 2.9 \\
& 3 & 0.058 & 2.8 \\
& 1 & 0.066 & 3.7 \\
\hline
\end{tabular}

A One-way ANOVA was performed on $\tau_{\varepsilon}$ and $\tau_{\sigma}$, followed by a Tukey post hoc test. The One-way ANOVA yielded a significant difference between the materials on both $\tau_{\varepsilon}$ and $\tau_{\sigma}$, as shown in Table 8 . The results of the Tukey test for $\tau_{\varepsilon}$ and $\tau_{\sigma}$ are presented on Tables 9 and 10, respectively. 
As shown in Table 9, Tukey's test for $\tau_{\varepsilon}$ yielded no significant difference between

1 PP fiber and Q. There was also no significant difference for $\tau_{\varepsilon}$ between for PU and Quatromer + 12 PP Fibers. There was a significant difference between Q and 1 PP fiber as compared to $\mathrm{PU}$ and $\mathrm{Q}+12 \mathrm{PP}$ fibers.

Table 7. Numerical summary of the creep test

\begin{tabular}{|l|cc|c|c|c|c|}
\hline \multicolumn{1}{|c|}{ Material } & $\eta_{\mathbf{1}}$ & $\mu_{\mathbf{0}}$ & $\mu_{\mathbf{1}}$ & $\mathbf{R}^{2}$ & $\tau_{\varepsilon}$ & $\tau_{\sigma}$ \\
\hline Quatromer (Q) & & & & & \\
Specimen 1 & 66.38 & 10.94 & 13.57 & 0.88 & 4.89 & 10.96 \\
Specimen 2 & 69.53 & 11.17 & 15.57 & 0.88 & 4.47 & 10.69 \\
Specimen 3 & 71.86 & 10.01 & 11.78 & 0.88 & 6.10 & 13.28 \\
\hline Mean & 69.26 & 10.71 & 13.64 & 0.88 & $\mathbf{5 . 1 5}$ & $\mathbf{1 1 . 6 4}$ \\
Std. Dev. & 2.75 & 0.62 & 1.89 & 0.00 & 0.85 & 1.42 \\
\hline PU & & & & & & \\
Specimen 1 & 79.47 & 12.36 & 4.07 & 0.87 & 19.54 & 25.97 \\
Specimen 2 & 68.58 & 13.35 & 4.01 & 0.81 & 17.12 & 22.25 \\
Specimen 3 & 75.47 & 11.61 & 3.70 & 0.89 & 20.41 & 26.91 \\
\hline Mean & 74.51 & 12.44 & 3.92 & 0.86 & 19.02 & $\mathbf{2 5 . 0 5}$ \\
Std. Dev. & 5.51 & 0.88 & 0.20 & 0.04 & 1.71 & 2.46 \\
\hline 1 PP Fiber & & & & & & \\
Specimen 1 & 51.95 & 7.87 & 12.05 & 0.88 & 4.31 & 10.91 \\
Specimen 2 & 46.86 & 8.48 & 11.97 & 0.86 & 3.92 & 9.44 \\
Specimen 3 & 54.94 & 9.24 & 9.26 & 0.85 & 5.93 & 11.88 \\
\hline Mean & 51.25 & 8.53 & 11.09 & 0.86 & $\mathbf{4 . 7 2}$ & 10.74 \\
Std. Dev. & 4.09 & 0.69 & 1.59 & 0.02 & 1.07 & 1.23 \\
\hline Quatromer + 12 PP Fibers & & & & & & \\
Specimen 1 & 63.67 & 7.31 & 2.95 & 0.90 & 21.58 & 30.29 \\
Specimen 2 & 78.42 & 8.94 & 3.62 & 0.86 & 21.66 & 30.43 \\
Specimen 3 & 79.59 & 9.34 & 3.75 & 0.86 & 21.20 & 29.73 \\
\hline Mean & 73.90 & 8.53 & 3.44 & 0.87 & $\mathbf{2 1 . 4 8}$ & $\mathbf{3 0 . 1 5}$ \\
Std. Dev. & 8.87 & 1.07 & 0.43 & 0.02 & 0.24 & 0.37 \\
\hline
\end{tabular}


Table 8. ANOVA for $\tau_{\varepsilon}$ (CSTRAIN) and $\tau_{\sigma}$ (CSTRESS)

\begin{tabular}{|ll|r|r|r|r|r|}
\hline & \multicolumn{1}{|c|}{ Sum of } & Squares & df & Mean Square & \multicolumn{1}{c|}{ F } & Sig. \\
\hline CSTRAIN & Getween Groups & 712.982 & 3 & 237.661 & 197.271 & .000 \\
& Within Groups & 9.638 & 8 & 1.205 & & \\
& Total & 722.620 & 11 & & & \\
\hline CSTRESS & Between Groups & 847.540 & 3 & 282.513 & 115.943 & .000 \\
& Within Groups & 19.493 & 8 & 2.437 & & \\
& Total & 867.033 & 11 & & & \\
\hline
\end{tabular}

Table 9. Tukey post hoc test for $\tau_{\varepsilon}$.

\section{Relaxation Time for Constant Strain}

Tukey HSD

\begin{tabular}{|l|r|r|r|}
\hline & & \multicolumn{2}{|c|}{ Subset for alpha $=.05$} \\
\cline { 3 - 4 } MATERIAL & $\mathrm{N}$ & 1 & \multicolumn{1}{c|}{2} \\
\hline 1 PP Fiber & 3 & 4.7200 & \\
Quatromer (Q) & 3 & 5.1533 & \\
PU & 3 & & 19.0233 \\
Q + 12 PP Fibers & 3 & & 21.4800 \\
Sig. & & .961 & .096 \\
\hline
\end{tabular}

Means for groups in homogeneous subsets are displayed.

a. Uses Hamonic Mean Sample Size $=3.000$.

Tukey's test for $\tau_{\sigma}$, indicated no significant difference between 1 PP fiber and Q (see

Table 10. However, unlike for $\tau_{\varepsilon}$, Tukey's test showed a significant difference between PU and Q + 12 PP fibers. 
Table 10. Tukey post hoc test for $\tau_{\sigma}$.

\begin{tabular}{l}
\hline \multicolumn{5}{|c|}{ Relaxation Time for Constant Stress } \\
\begin{tabular}{|l|r|r|r|r|}
\hline Tukey HSD & \multicolumn{3}{|c|}{ Subset for alpha $=.05$} \\
\cline { 2 - 5 } MATERIAL & $\mathrm{N}$ & 1 & 2 & 3 \\
\hline 1 PP Fiber & 3 & 10.7433 & & \\
Quatromer (Q) & 3 & 11.6433 & & \\
PU & 3 & & 25.0433 & \\
Q + 12 PP Fibers & 3 & & & 30.1500 \\
Sig. & & .892 & 1.000 & 1.000 \\
\hline
\end{tabular} \\
Means for groups in homogeneous subsets are displayed. \\
a. Uses Harmonic Mean Sample Size $=3.000$. \\
\hline
\end{tabular}

\subsubsection{Relaxation}

Relaxation tests were performed in order to characterize the response of a body when it is suddenly strained and then the strain is maintained constant afterwards. Normalized results for the complete relaxation test are presented in Figure 23. Three specimens of each material were tested. Figure 24 shows the initial 20 seconds of the test, where the initial response is shown clearly. Figure 25 shows the last 80 seconds of the test, where the final relaxation occurs.

As seen on Figure 23, Quatromer showed the highest decay of stress. PU showed the lowest decay of stress. Q +12 PP fibers and 1 PP fiber fall in between. The same procedures used to analyze the results of the creep test were used to analyze the relaxation results. It was not possible to fit a single curve to the data so the data were divided in two parts. The first portion of the curve included the upper $63.2 \%$ of the data points of the total relaxation (Figure 24). This is where most of the decay of stress occurs in the less amount of time. The second portion of the cure includes the lower $36.8 \%$ of 
the data points of the total relaxation (Figure 25), where the decay of stress is much lower when compared to the initial part of the curve.

As shown in Figure 24, there was a clear difference between Q + 12 PP fibers and PU. PU had the lowest decay of stress. The rate of decay of the PP fiber is somewhat in the ranges of that for PU and Q +12 PP fibers

In Figure 25, the last 80 seconds of the relaxation test is presented. The four materials had the same rate of decay at this stage of the test. Quatromer still had the highest decay of stress, while PU had the lowest decay of stress. Q + 12 PP fibers and the 1 PP fiber fell in between the other two materials.

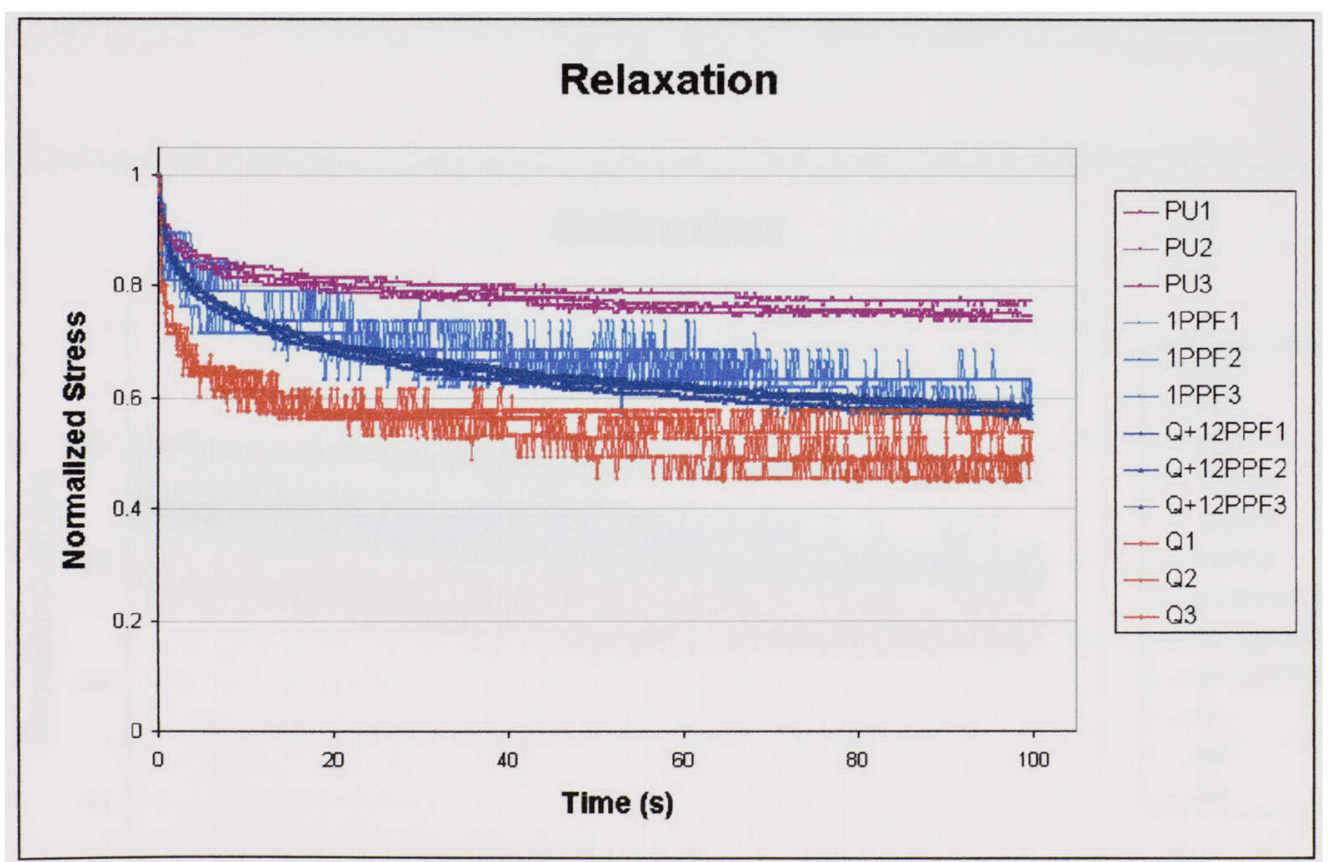

Figure 23. Normalized stress relaxation plotted against time 


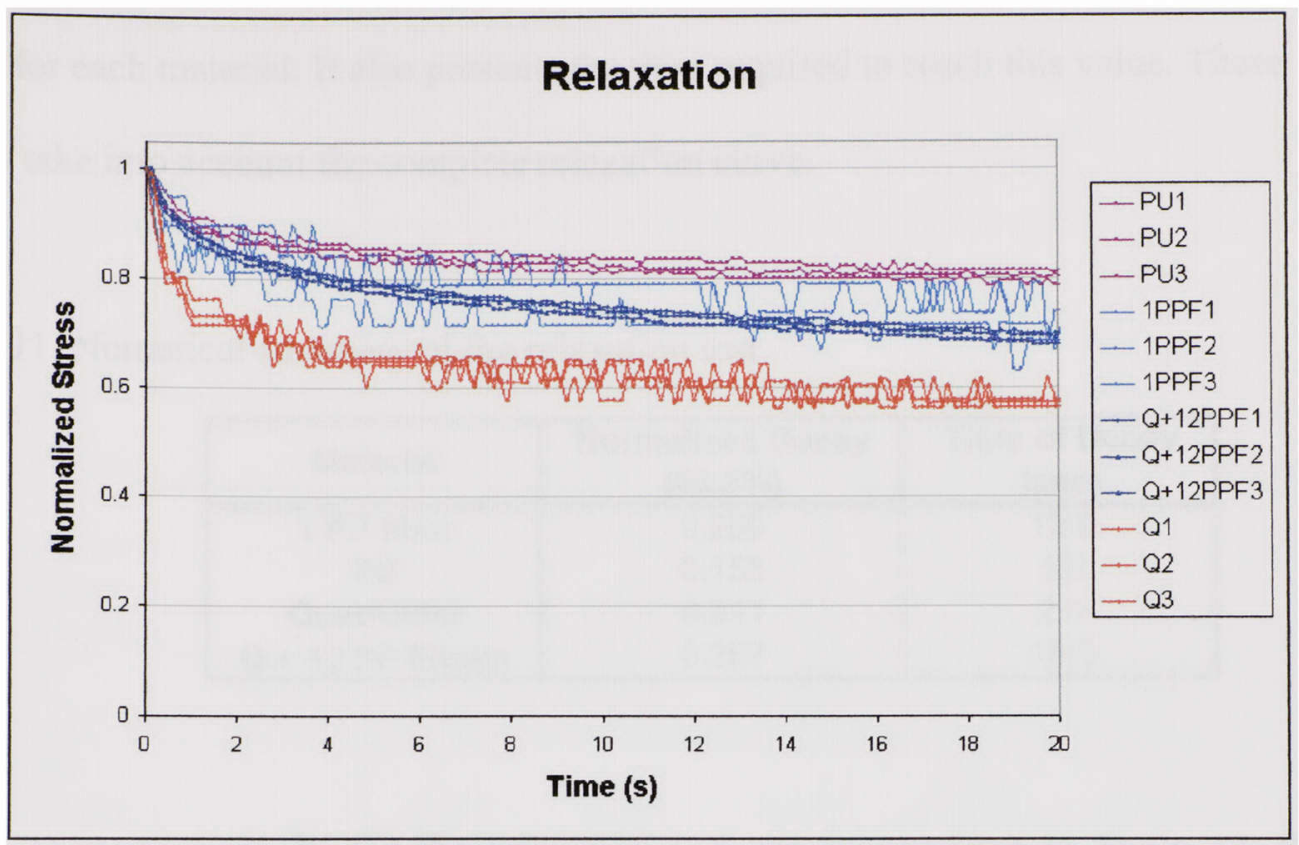

Figure 24. Normalized stress relaxation plotted against time (first 20 seconds)

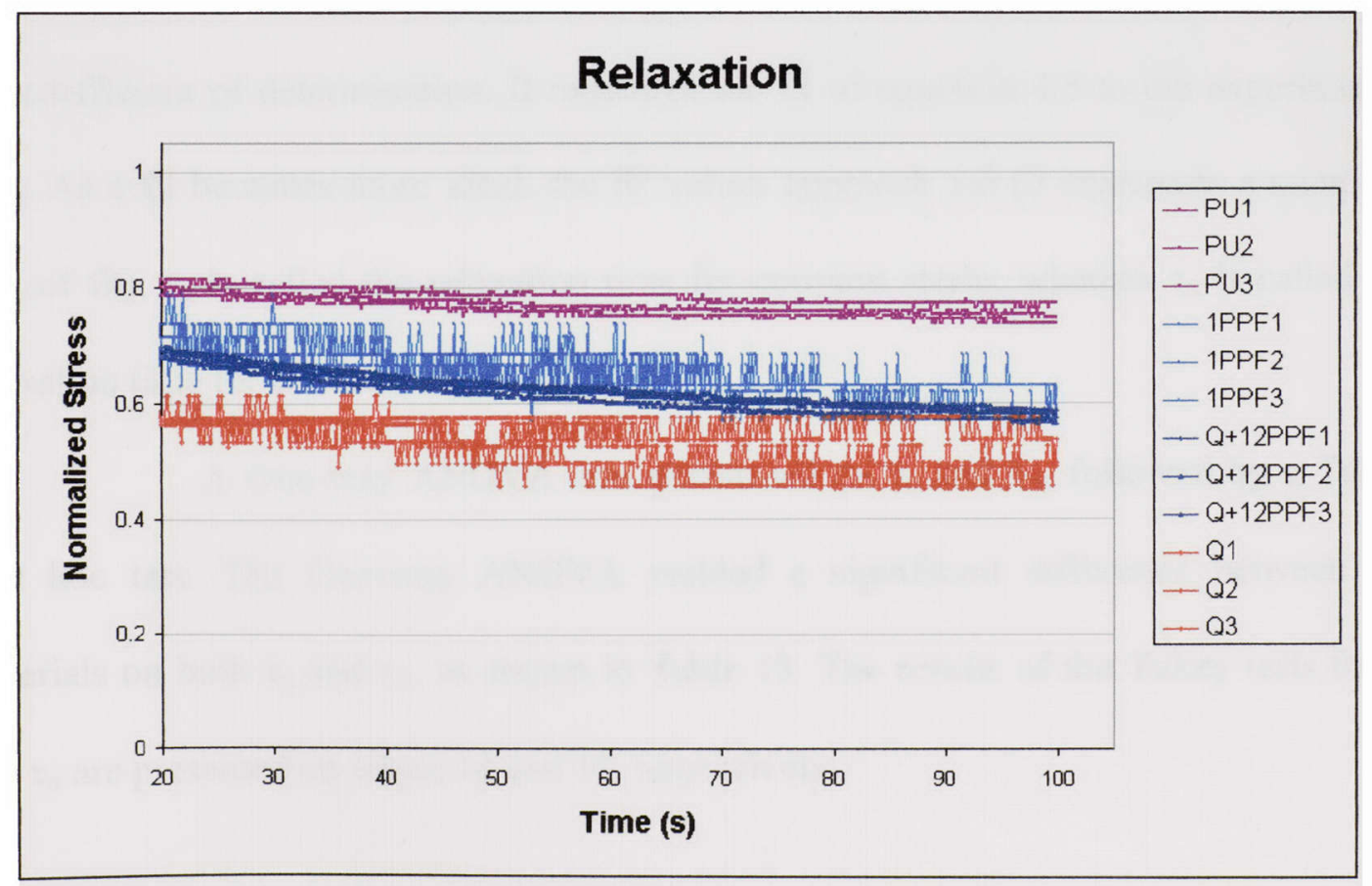

Figure 25. Normalized stress relaxation plotted against time (Last 80 seconds) 
Table 11 presents the values of the normalized amount of the upper $63.2 \%$ of decay for each material. It also presents the time required to reach this value. These results take into account the complete relaxation curve.

Table 11. Numerical summary of the relaxation test

\begin{tabular}{|c|c|c|}
\hline Material & $\begin{array}{c}\text { Normalized Decay } \\
(\mathbf{6 3 . 2 \% )}\end{array}$ & $\begin{array}{c}\text { Time of Decay } \\
\text { (sec) }\end{array}$ \\
\hline 1 PP fiber & 0.250 & 12.0 \\
PU & 0.155 & 5.0 \\
Quatromer & 0.311 & 2.1 \\
Q + 12 PP Fibers & 0.267 & 15.3 \\
\hline
\end{tabular}

Numerical results for the relaxation test are summarized in Table 12. Part a) shows the results for the upper $63.2 \%$ of the curve, while part b) shows the results of the lower $36.8 \%$ of the curve. Here, $\mu_{0}, \mu_{1}$, and $\eta_{1}$ are constants used in Equation $4.5 . \mathrm{R}^{2}$ is the coefficient of determination. It measures the fit of equation 4.8 to the experimental data. As a fit becomes more ideal, the $\mathrm{R}^{2}$ values approach 1.0 ( 0 represents a complete lack of fit). $\tau_{\varepsilon}$ is called the relaxation time for constant strain, whereas $\tau_{\sigma}$ is called the relaxation time for constant stress.

A One-way ANOVA was performed on $\tau_{\varepsilon}$ and $\tau_{\sigma}$, followed by a Tukey post hoc test. The One-way ANOVA yielded a significant difference between the materials on both $\tau_{\varepsilon}$ and $\tau_{\sigma}$, as shown in Table 13. The results of the Tukey tests for $\tau_{\varepsilon}$ and $\tau_{\sigma}$ are presented on tables 14 and 15 , respectively. 
Table 12. Numerical summary of the relaxation test a) upper $63.2 \%$ of the curve, b) lower $36.8 \%$ of the curve.

\begin{tabular}{|c|c|c|c|c|c|c|}
\hline Material & $\eta_{1}$ & $\mu_{0}$ & $\mu_{1}$ & $\mathbf{R}^{2}$ & $\tau_{\varepsilon}$ & $\tau_{\sigma}$ \\
\hline \multicolumn{7}{|l|}{ Quatromer } \\
\hline Specimen 1 & 0.057 & 0.179 & 0.093 & 0.929 & 0.61 & 0.93 \\
\hline Specimen 2 & 0.070 & 0.179 & 0.088 & 0.937 & 0.80 & 1.19 \\
\hline Specimen 3 & 0.052 & 0.177 & 0.076 & 0.926 & 0.68 & 0.98 \\
\hline Mean & 0.060 & 0.178 & 0.086 & 0.931 & 0.698 & 1.032 \\
\hline Std. Dev. & 0.009 & 0.001 & 0.009 & 0.006 & 0.093 & 0.137 \\
\hline \multicolumn{7}{|l|}{ PU } \\
\hline Specimen 1 & 0.308 & 1.137 & 0.176 & 0.884 & 1.75 & 2.02 \\
\hline Specimen 2 & 0.366 & 1.225 & 0.187 & 0.955 & 1.96 & 2.26 \\
\hline Specimen 3 & 0.478 & 1.544 & 0.255 & 0.960 & 1.87 & 2.18 \\
\hline Mean & 0.384 & 1.302 & 0.206 & 0.933 & 1.860 & 2.153 \\
\hline Std. Dev. & 0.087 & 0.214 & 0.043 & 0.043 & 0.107 & 0.123 \\
\hline \multicolumn{7}{|l|}{1 PP Fiber } \\
\hline Specimen 1 & 0.092 & 0.151 & 0.046 & 0.846 & 1.99 & 2.60 \\
\hline Specimen 2 & 0.059 & 0.150 & 0.038 & 0.811 & 1.54 & 1.94 \\
\hline Specimen 3 & 0.081 & 0.149 & 0.038 & 0.840 & 2.12 & 2.66 \\
\hline Mean & 0.078 & 0.150 & 0.041 & 0.832 & 1.882 & 2.399 \\
\hline Std. Dev. & 0.017 & 0.001 & 0.005 & 0.018 & 0.300 & 0.400 \\
\hline \multicolumn{7}{|l|}{ Quatromer + 12 PP Fibers } \\
\hline Specimen 1 & 3.006 & 1.888 & 0.600 & 0.974 & 5.01 & 6.61 \\
\hline Specimen 2 & 2.682 & 1.740 & 0.606 & 0.967 & 4.43 & 5.97 \\
\hline Specimen 3 & 3.160 & 1.684 & 0.538 & 0.929 & 5.87 & 7.75 \\
\hline Mean & 2.949 & 1.771 & 0.581 & 0.957 & 5.105 & 6.774 \\
\hline Std. Dev. & 0.244 & 0.106 & 0.037 & 0.024 & 0.727 & 0.902 \\
\hline
\end{tabular}

a) 


\begin{tabular}{|c|c|c|c|c|c|c|}
\hline Material & $\eta_{1}$ & $\mu_{0}$ & $\mu_{1}$ & $\mathbf{R}^{2}$ & $\tau_{\varepsilon}$ & $\tau_{\sigma}$ \\
\hline \multicolumn{7}{|l|}{ Quatromer } \\
\hline Specimen 1 & 1.745 & 0.128 & 0.057 & 0.863 & 30.62 & 44.22 \\
\hline Specimen 2 & 1.766 & 0.122 & 0.071 & 0.856 & 24.93 & 39.42 \\
\hline Specimen 3 & 1.659 & 0.135 & 0.059 & 0.813 & 28.01 & 40.33 \\
\hline Mean & 1.723 & 0.128 & 0.062 & 0.844 & 27.852 & 41.325 \\
\hline Std. Dev. & 0.057 & 0.006 & 0.007 & 0.027 & 2.844 & 2.551 \\
\hline \multicolumn{7}{|l|}{$\mathrm{PU}$} \\
\hline Specimen 1 & 6.697 & 1.055 & 0.179 & 0.911 & 37.51 & 43.86 \\
\hline Specimen 2 & 7.647 & 1.119 & 0.178 & 0.918 & 43.01 & 49.84 \\
\hline Specimen 3 & 8.106 & 1.377 & 0.234 & 0.924 & 34.70 & 40.58 \\
\hline Mean & 7.483 & 1.183 & 0.197 & 0.918 & 38.403 & 44.761 \\
\hline Std. Dev. & 0.718 & 0.170 & 0.032 & 0.007 & 4.227 & 4.694 \\
\hline \multicolumn{7}{|l|}{1 PP Fiber } \\
\hline Specimen 1 & 2.041 & 0.114 & 0.048 & 0.834 & 42.18 & 60.04 \\
\hline Specimen 2 & 1.941 & 0.119 & 0.047 & 0.821 & 40.99 & 57.26 \\
\hline Specimen 3 & 1.853 & 0.116 & 0.051 & 0.857 & 36.41 & 52.34 \\
\hline Mean & 1.945 & 0.117 & 0.049 & 0.838 & 39.860 & 56.547 \\
\hline Std. Dev. & 0.094 & 0.003 & 0.002 & 0.018 & 3.045 & 3.899 \\
\hline \multicolumn{7}{|l|}{ Quatromer + 12 PP Fibers } \\
\hline Specimen 1 & 26.364 & 1.490 & 0.724 & 0.957 & 36.41 & 54.11 \\
\hline Specimen 2 & 24.960 & 1.351 & 0.681 & 0.954 & 36.63 & 55.10 \\
\hline Specimen 3 & 23.862 & 1.341 & 0.646 & 0.958 & 36.93 & 54.72 \\
\hline$\overline{\text { Mean }}$ & 25.062 & 1.394 & 0.684 & 0.957 & 36.658 & 54.645 \\
\hline Std. Dev. & 1.254 & 0.083 & 0.039 & 0.002 & 0.257 & 0.500 \\
\hline
\end{tabular}

b)

Table 13. ANOVA for $\tau_{\varepsilon}\left(\right.$ CSTRAIN) and $\tau_{\sigma}$ (CSTRESS); a) upper $63.2 \%$, b) lower $36.8 \%$ of the curve.

\begin{tabular}{|ll|r|r|r|r|r|}
\hline & Sum of & df & Mean Square & F & Sig. \\
\hline TSTRAIN & Between Groups & 32.301 & 3 & 10.767 & 67.516 & .000 \\
& Mithin Groups & 1.276 & 8 & .159 & & \\
& Total & 33.577 & 11 & & & \\
\hline TSTRESS Between Groups & 57.524 & 3 & 19.175 & 76.204 & .000 \\
& Within Groups & 2.013 & 8 & .252 & & \\
& Total & 59.537 & 11 & & & \\
\hline
\end{tabular}

a) 


\begin{tabular}{|c|c|c|c|c|c|c|}
\hline & & $\begin{array}{l}\text { Sum of } \\
\text { Squares }\end{array}$ & $d f$ & Mean Square & $F$ & Sig. \\
\hline \multirow[t]{3}{*}{ TSTRAIN } & Between Groups & 261.351 & 3 & \multirow{3}{*}{$\begin{array}{r}87.117 \\
8.832\end{array}$} & \multirow[t]{3}{*}{9.864} & \multirow[t]{3}{*}{.005} \\
\hline & Within Groups & 70.657 & 8 & & & \\
\hline & Total & 332.008 & 11 & & & \\
\hline \multirow[t]{3}{*}{ TSTRESS } & Between Groups & 495.909 & 3 & \multirow{3}{*}{$\begin{array}{r}165.303 \\
11.000\end{array}$} & \multirow[t]{3}{*}{15.028} & \multirow[t]{3}{*}{.001} \\
\hline & Within Groups & 87.996 & 8 & & & \\
\hline & Total & 583.905 & 11 & & & \\
\hline
\end{tabular}

b)

In Table 14, Tukey's test for $\tau_{\varepsilon}$ showed there was no significant difference between 1 PP fiber and PU. Quatromer was significantly different than these two materials as well as $Q+12$ PP fibers, which had the highest mean for $\tau_{\varepsilon}$. There was also no significant difference between $\tau_{\varepsilon}$ for PU and Q +12 PP fibers. Table 15 shows the same distribution of results for $\tau_{\sigma}$ as for $\tau_{\varepsilon}$.

Table 14. Tukey post hoc test for $\tau_{\varepsilon}$ (upper $63.2 \%$ of the curve,). Mean values are shown.

\section{Relaxation Time for Constant Strain}

Tukey HSD ${ }^{a}$

\begin{tabular}{|l|r|r|r|r|}
\hline \multirow{2}{*}{ TEST } & \multirow{2}{*}{$N$} & \multicolumn{3}{|c|}{ Subset for alpha $=.05$} \\
\cline { 3 - 5 } & \multicolumn{1}{|c|}{1} & \multicolumn{1}{c|}{3} \\
\hline Quatromer (Q) & 3 & .6967 & & \\
PU & 3 & & 1.8600 & \\
1 PP Fiber & 3 & & 1.8833 & \\
Q + 12 PP Fibers & 3 & & & 5.1033 \\
Sig. & & 1.000 & 1.000 & 1.000 \\
\hline
\end{tabular}

Means for groups in homogeneous subsets are displayed.

a. Uses Harmonic Mean Sample Size $=3.000$. 
Table 15 . Tukey post hoc test for $\tau_{\sigma}$ (upper $63.2 \%$ of the curve). Mean values are shown.

Relaxation Time for Constant Stress

Tukey HSD $^{a}$
\begin{tabular}{|l|r|r|r|r|}
\hline & & \multicolumn{3}{|c|}{ Subset for alpha $=.05$} \\
\cline { 3 - 5 } TEST & $N$ & 1 & 2 & 3 \\
\hline Quatromer (Q) & 3 & 1.0333 & & \\
PU & 3 & & 2.1533 & \\
1 PP Fiber & 3 & & 2.4000 & \\
Q + 12 PP Fiber & 3 & & & 6.7767 \\
Sig. & & .097 & .929 & 1.000 \\
\hline
\end{tabular}

Means for groups in homogeneous subsets are displayed.

a. Uses Harmonic Mean Sample Size $=3.000$.

\subsubsection{Hysteresis}

Hysteresis tests were performed in order to present the phenomenon when a body that is subjected to cyclic loading has a different stress-strain relationship in the loading process than in the unloading process. Three specimens of each material were tested. Figure 26 shows the results of the hysteresis test for Quatromer, PU, and the Q +12 PP fibers at $1.25 \mathrm{~Hz}$. Of the three polyurethane specimens one was damaged before the test, only two specimens were tested. Figure 27 shows the results of the hysteresis test for the PP fibers. This test is shown in a separate chart due to the large difference in stress levels involved. The results of the hysteresis tests of all the materials are presented in a normalized chart as shown in Figure 28. 


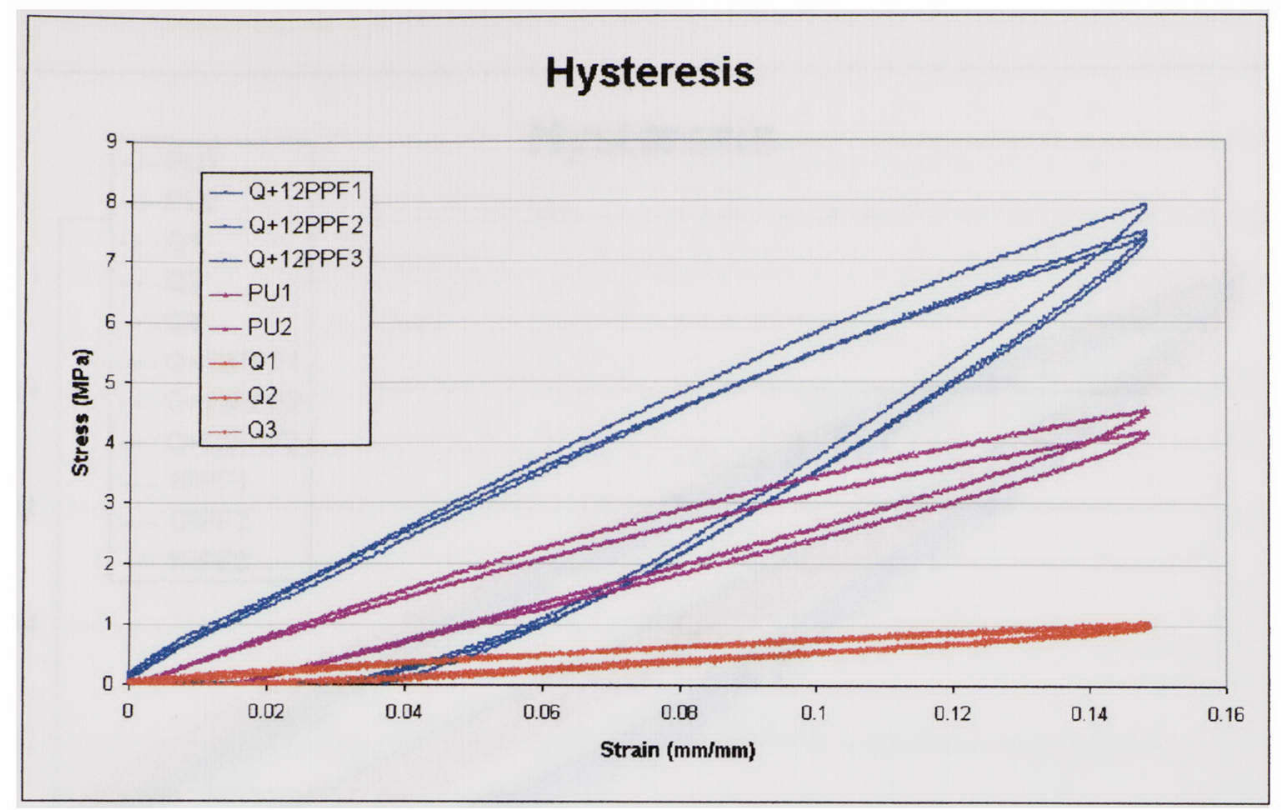

Figure 26. Hysteresis test results at $1.25 \mathrm{~Hz}$

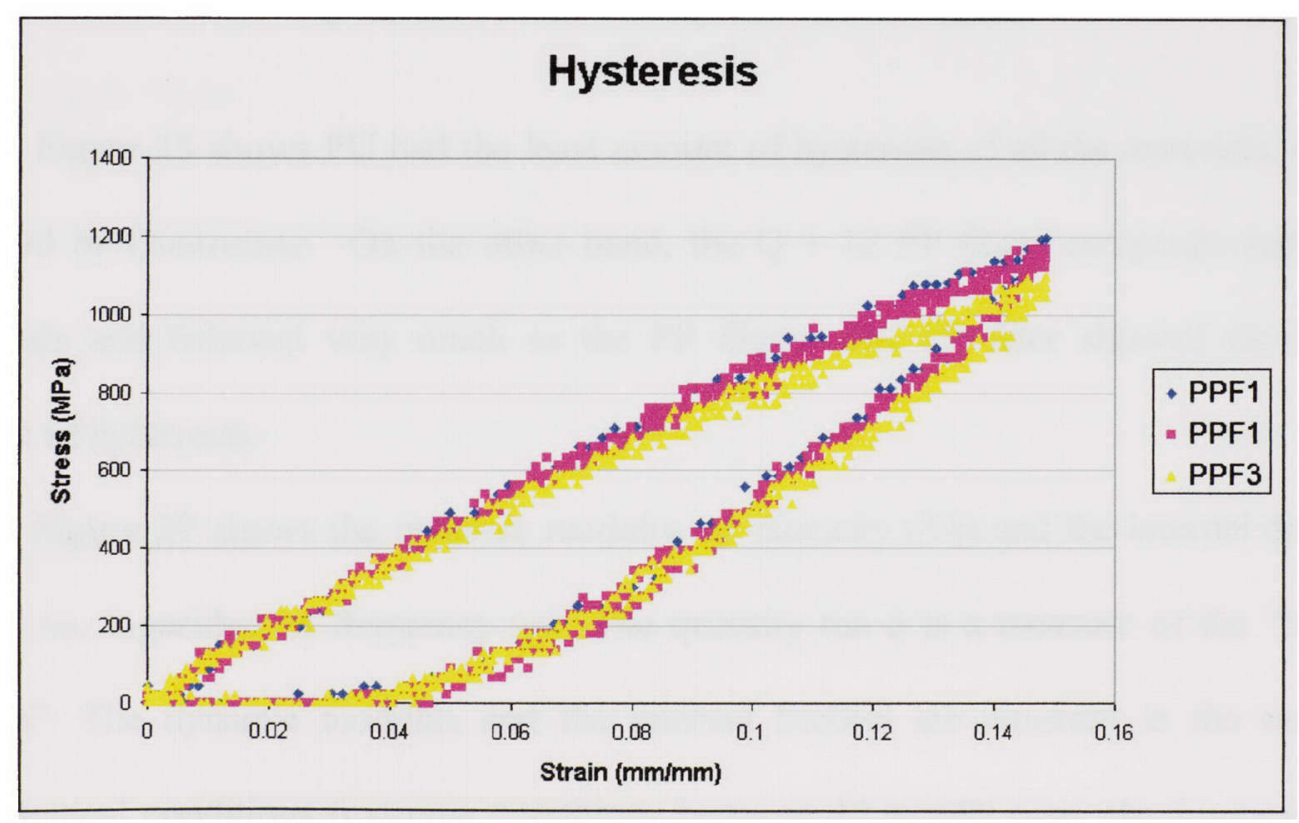

Figure 27. Hysteresis test results at $1.25 \mathrm{~Hz}$ 


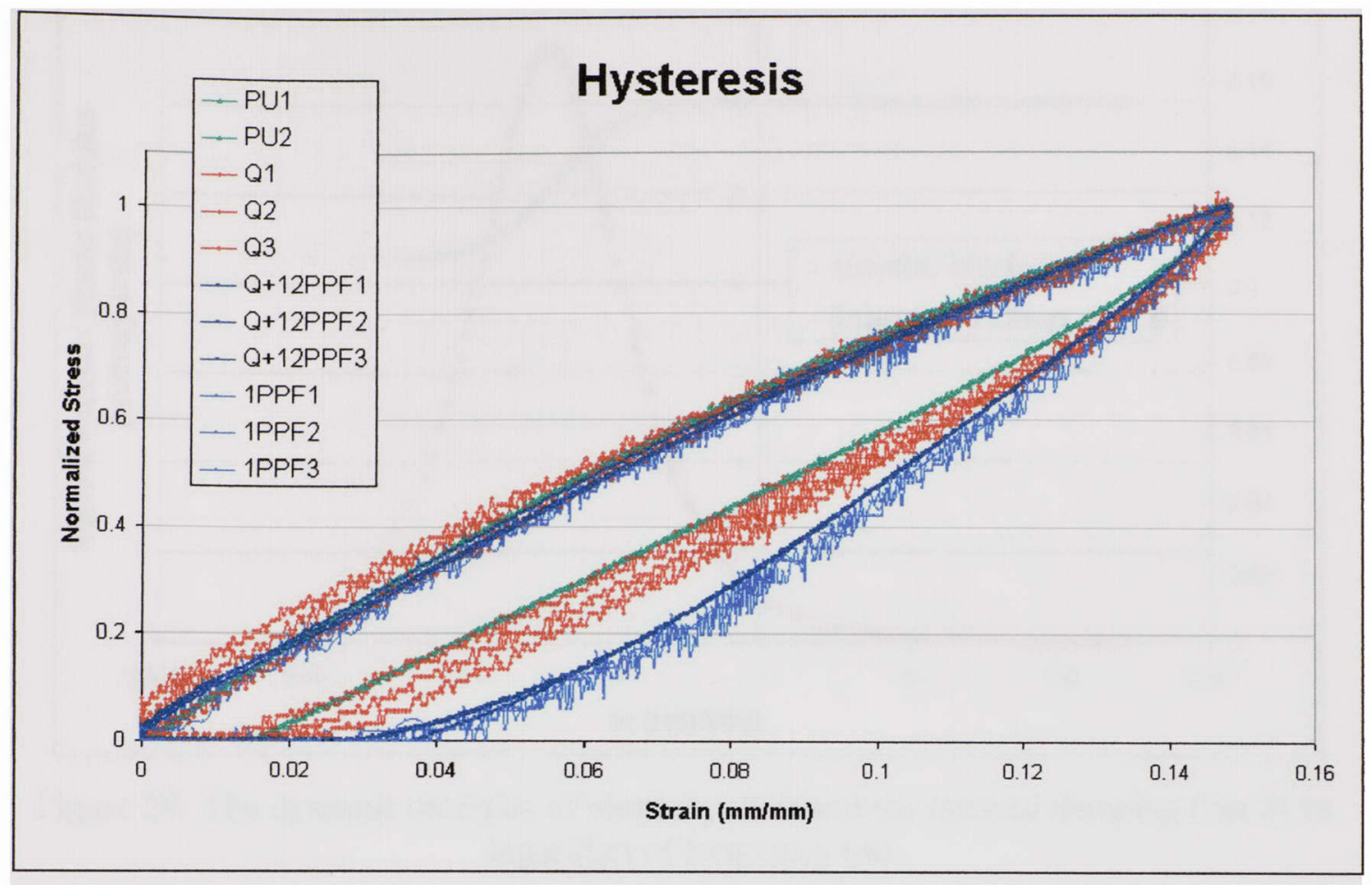

Figure 28. Normalized hysteresis test results

Figure 28 shows PU had the least amount of hysteresis of all the materials, closely followed by Quatromer. On the other hand, the Q + 12 PP fibers composite had more hysteresis and behaved very much as the PP fibers. The PP fiber showed the largest amount of hysteresis.

Figure 29 shows the dynamic modulus of elasticity $(|\mathrm{G}|)$ and the internal damping $(\tan \delta)$ vs. logarithm of frequency $(\omega)$. The quantity $\tan \delta$ is a measure of the "internal friction". The dynamic modulus and the internal friction are constant in the range of physiological conditions (extreme conditions between 40 to $180 \mathrm{bpm}$, the frequency $(\omega)$ is equivalent to 4.2 to $18.9 \mathrm{rad} / \mathrm{sec}$ ), see Figure 29. 


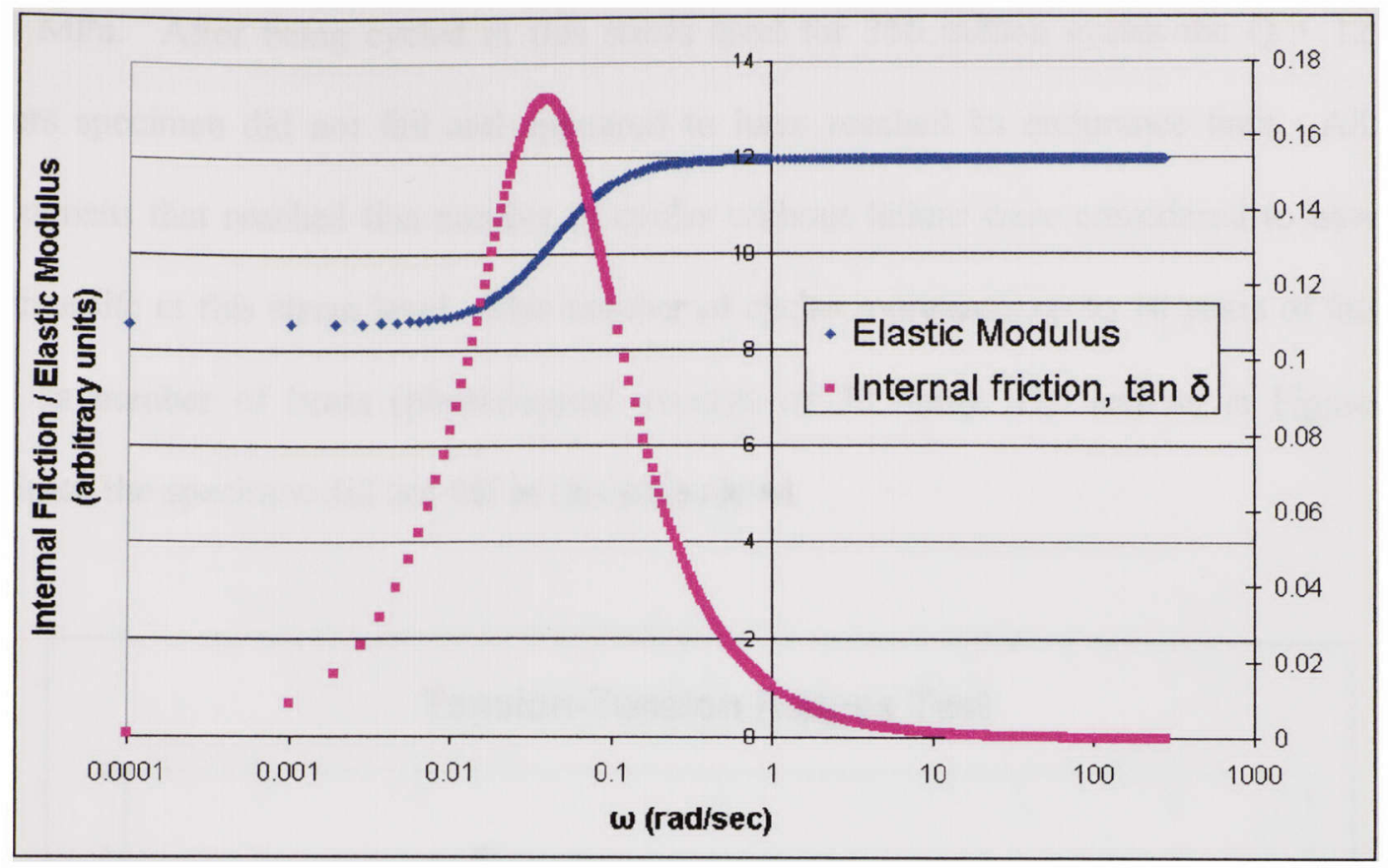

Figure 29. The dynamic modulus of elasticity $(|\mathrm{G}|)$ and the internal damping $(\tan \delta)$ vs. logarithm of frequency $(\omega)$

\subsection{Dynamic Tests}

\subsubsection{Tension-Tension Fatigue Test}

Tension-tension fatigue tests were performed in order to create a stress vs. number of cycles curve. Results are presented in Figures 30 and 31. This figure shows the stress vs. number of cycles for Quatromer, Q + 3 PP fibers, PU, and Q + 12 PP fibers.

Quatromer showed an endurance limit below 0.33 MPa. At this stress level the specimen failed after 130 million cycles, equivalent to 3.5 years at physiological rate of 70 bpm. Q + 3 PP fibers showed an endurance limit higher than for Quatromer. PU had a higher endurance limit than the previous two materials, above 1.5 MPa. The specimen did not fail at this stress level. Q +12 PP fibers showed an endurance limit above PU, at 
2.5 MPa. After being cycled at this stress level for 350 million cycles the Q $+12 \mathrm{PP}$ fibers specimen did not fail and appeared to have reached its endurance limit. All the specimens that reached this number of cycles without failure were considered to have an infinite life at this stress level. This number of cycles represents up to 10 years of fatigue life as number of beats (physiological average of $70 \mathrm{bpm}$ ). The arrows in Figure 30 indicate the specimen did not fail at this stress level.

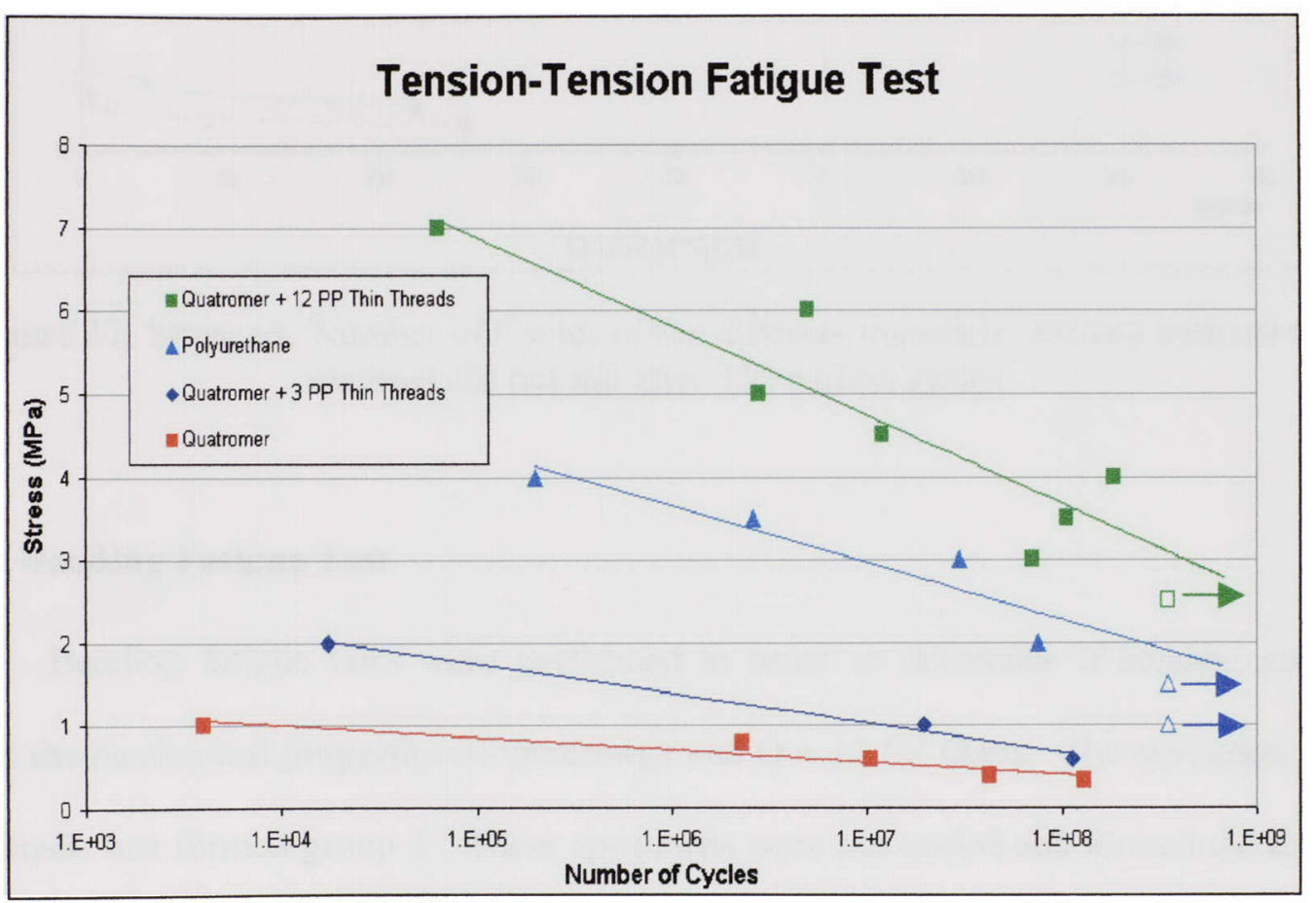

Figure 30. Stress vs. Number of Cycles of the different materials on a semi-log scale. Arrows indicate the material did not fail after 350 million cycles 


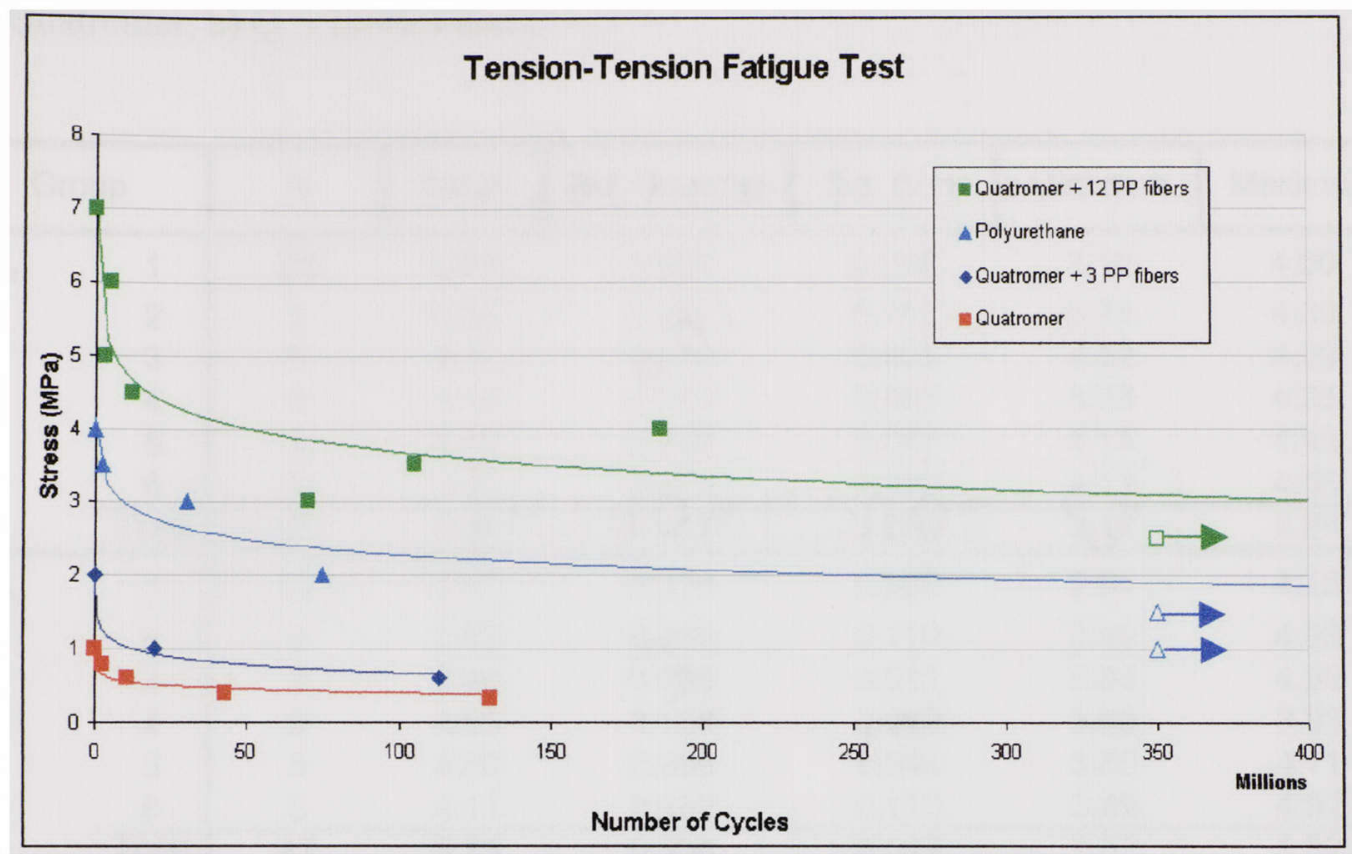

Figure 31. Stress vs. Number of Cycles of the different materials. Arrows indicate the material did not fail after 350 million cycles

\subsubsection{Bending Fatigue Test}

Bending fatigue tests were performed in order to determine if bending stresses affect the mechanical properties of Quatromer and Q +12 PP fibers. The specimens from the tensile test formed group 1 . These specimens were not cycled and formed the control group. Groups 2, 3, 4, 5, and 6 were cycles for 7, 16, 57.5, 79, and 89 million cycles, respectively. As mentioned before, each group contains five specimens of Quatromer, and five specimens of Q + 12 PP fibers. Results of six groups were compared. Tables $16 \mathrm{a}$ and $16 \mathrm{~b}$ present the results of the tensile test for the Quatromer and Q $+12 \mathrm{PP}$ fibers, respectively. 
Table 16. Numerical summary of the tensile results of the bending fatigue test a) Quatromer, b) Q + 12 PP Fibers.

\begin{tabular}{|c|c|c|c|c|c|c|c|}
\hline \multicolumn{2}{|c|}{ Group } & $N$ & Mean & Std. Deviation & Std. Error & Minimum & Maximum \\
\hline \multirow[t]{7}{*}{$\mathrm{E}_{\text {true }}$} & 1 & 22 & 3.88 & 0.405 & 0.086 & 3.12 & 4.60 \\
\hline & 2 & 5 & 4.16 & 0.262 & 0.117 & 3.74 & 4.39 \\
\hline & 3 & 5 & 4.17 & 0.070 & 0.032 & 4.12 & 4.29 \\
\hline & 4 & 5 & 4.10 & 0.145 & 0.065 & 3.93 & 4.25 \\
\hline & 5 & 5 & 4.30 & 0.105 & 0.047 & 4.14 & 4.42 \\
\hline & 6 & 5 & 4.41 & 0.275 & 0.123 & 4.13 & 4.86 \\
\hline & Total & 47 & 4.06 & 0.359 & 0.052 & 3.12 & 4.86 \\
\hline \multirow[t]{7}{*}{$\mathrm{E}_{\text {eng }}$} & 1 & 22 & 3.61 & 0.376 & 0.080 & 2.91 & 4.28 \\
\hline & 2 & 5 & 3.87 & 0.245 & 0.110 & 3.49 & 4.09 \\
\hline & 3 & 5 & 3.88 & 0.068 & 0.031 & 3.83 & 4.00 \\
\hline & 4 & 5 & 3.80 & 0.138 & 0.062 & 3.63 & 3.93 \\
\hline & 5 & 5 & 4.00 & 0.098 & 0.044 & 3.85 & 4.11 \\
\hline & 6 & 5 & 4.11 & 0.252 & 0.113 & 3.85 & 4.52 \\
\hline & Total & 47 & 3.78 & 0.334 & 0.049 & 2.91 & 4.52 \\
\hline \multirow[t]{7}{*}{$\mathrm{UTS}_{\text {true }}$} & 1 & 22 & 1.43 & 0.154 & 0.033 & 1.20 & 1.81 \\
\hline & 2 & 5 & 1.73 & 0.075 & 0.033 & 1.60 & 1.78 \\
\hline & 3 & 5 & 1.70 & 0.042 & 0.019 & 1.64 & 1.74 \\
\hline & 4 & 5 & 1.71 & 0.065 & 0.029 & 1.62 & 1.79 \\
\hline & 5 & 5 & 1.77 & 0.051 & 0.023 & 1.71 & 1.82 \\
\hline & 6 & 5 & 1.76 & 0.113 & 0.051 & 1.63 & 1.94 \\
\hline & Total & 47 & 1.59 & 0.194 & 0.028 & 1.20 & 1.94 \\
\hline \multirow[t]{7}{*}{ UTS $_{\text {eng }}$} & 1 & 22 & 0.95 & 0.102 & 0.022 & 0.80 & 1.21 \\
\hline & 2 & 5 & 1.16 & 0.051 & 0.023 & 1.07 & 1.19 \\
\hline & 3 & 5 & 1.14 & 0.025 & 0.011 & 1.10 & 1.16 \\
\hline & 4 & 5 & 1.15 & 0.040 & 0.018 & 1.09 & 1.19 \\
\hline & 5 & 5 & 1.18 & 0.036 & 0.016 & 1.14 & 1.22 \\
\hline & 6 & 5 & 1.18 & 0.070 & 0.031 & 1.10 & 1.29 \\
\hline & Total & 47 & 1.06 & 0.130 & 0.019 & 0.80 & 1.29 \\
\hline
\end{tabular}

a) 


\begin{tabular}{|c|c|c|c|c|c|c|c|}
\hline & & $N$ & Mean & Std. Deviation & Std. Error & Minimum & Maximum \\
\hline \multirow[t]{7}{*}{ US } & 1 & 10 & 0.30 & 0.026 & 0.008 & 0.25 & 0.35 \\
\hline & 2 & 4 & 0.32 & 0.013 & 0.006 & 0.31 & 0.34 \\
\hline & 3 & 5 & 0.34 & 0.052 & 0.023 & 0.28 & 0.41 \\
\hline & 4 & 5 & 0.34 & 0.019 & 0.008 & 0.31 & 0.36 \\
\hline & 5 & 5 & 0.34 & 0.032 & 0.014 & 0.30 & 0.38 \\
\hline & 6 & 5 & 0.34 & 0.024 & 0.011 & 0.30 & 0.36 \\
\hline & Total & 34 & 0.33 & 0.032 & 0.005 & 0.25 & 0.41 \\
\hline \multirow[t]{7}{*}{$\mathrm{E}_{\text {true }}$} & 1 & 10 & 42.80 & 4.856 & 1.535 & 35.95 & 49.18 \\
\hline & 2 & 4 & 44.72 & 1.950 & 0.975 & 42.51 & 46.68 \\
\hline & 3 & 5 & 41.71 & 1.698 & 0.760 & 40.59 & 44.68 \\
\hline & 4 & 5 & 41.87 & 1.523 & 0.681 & 40.44 & 43.91 \\
\hline & 5 & 5 & 44.82 & 5.311 & 2.375 & 40.75 & 54.01 \\
\hline & 6 & 5 & 45.77 & 2.806 & 1.255 & 42.77 & 49.21 \\
\hline & Total & 34 & 43.46 & 3.748 & 0.643 & 35.95 & 54.01 \\
\hline \multirow[t]{7}{*}{$\mathrm{E}_{\text {eng }}$} & 1 & 10 & 39.84 & 4.519 & 1.429 & 33.45 & 45.77 \\
\hline & 2 & 4 & 41.63 & 1.819 & 0.910 & 39.57 & 43.46 \\
\hline & 3 & 5 & 38.83 & 1.572 & 0.703 & 37.79 & 41.58 \\
\hline & 4 & 5 & 38.97 & 1.420 & 0.635 & 37.64 & 40.87 \\
\hline & 5 & 5 & 41.72 & 4.941 & 2.210 & 37.94 & 50.27 \\
\hline & 6 & 5 & 42.61 & 2.613 & 1.169 & 39.81 & 45.81 \\
\hline & Total & 34 & 40.46 & 3.488 & 0.598 & 33.45 & 50.27 \\
\hline \multirow[t]{7}{*}{$\mathrm{UTS}_{\text {true }}$} & 1 & 10 & 9.74 & 1.149 & 0.363 & 8.18 & 11.26 \\
\hline & 2 & 4 & 10.22 & 0.550 & 0.275 & 9.54 & 10.82 \\
\hline & 3 & 5 & 10.24 & 1.008 & 0.451 & 8.81 & 11.30 \\
\hline & 4 & 5 & 10.08 & 0.659 & 0.295 & 9.45 & 11.14 \\
\hline & 5 & 5 & 10.73 & 1.129 & 0.505 & 9.53 & 12.39 \\
\hline & 6 & 5 & 10.74 & 0.943 & 0.422 & 9.41 & 11.52 \\
\hline & Total & 34 & 10.21 & 0.990 & 0.170 & 8.18 & 12.39 \\
\hline \multirow[t]{7}{*}{ UTS $_{\text {eng }}$} & 1 & 10 & 7.47 & 0.817 & 0.259 & 6.36 & 8.38 \\
\hline & 2 & 4 & 7.71 & 0.345 & 0.172 & 7.28 & 8.05 \\
\hline & 3 & 5 & 7.65 & 0.611 & 0.273 & 6.87 & 8.46 \\
\hline & 4 & 5 & 7.55 & 0.383 & 0.171 & 7.32 & 8.22 \\
\hline & 5 & 5 & 8.04 & 0.901 & 0.403 & 7.30 & 9.54 \\
\hline & 6 & 5 & 8.02 & 0.576 & 0.257 & 7.25 & 8.51 \\
\hline & Total & 34 & 7.70 & 0.669 & 0.115 & 6.36 & 9.54 \\
\hline
\end{tabular}

b)

For Quatromer, a One-way ANOVA was performed to compare differences between groups in the following parameters: True Young's modulus $\left(\mathrm{E}_{\text {true }}\right)$, Engineering 
Young's modulus ( $\left.E_{\text {eng }}\right)$, True Ultimate Tensile Stress $\left(\mathrm{UTS}_{\text {true }}\right)$, and Engineering Ultimate Tensile Stress $\left(\mathrm{UTS}_{\text {eng }}\right)$. Results are presented in Table 17. This table shows there is a weak significant difference between the groups for $E_{\text {true }}$ and $E_{\text {eng. }}$. On the other hand, Table 17 shows there was a significant difference between groups for the $\mathrm{UTS}_{\text {true }}$ and UTS $_{\text {eng. }}$.

A Tukey post hoc test was performed for each of the groups in order to find out the differences between each group. For $E_{\text {true }}$ and $E_{\text {eng }}$ there was no significant difference between groups as shown in tables 18 and 19 , respectively.

Table 17. One-way ANOVA results for all Quatromer groups

\begin{tabular}{|ll|r|r|r|r|r|}
\hline & $\begin{array}{r}\text { Sum of } \\
\text { Squares }\end{array}$ & df & Mean Square & \multicolumn{1}{c|}{$F$} & Sig. \\
\hline ETRUE & Between Groups & 1.763 & 5 & .353 & 3.468 & .011 \\
& Within Groups & 4.169 & 41 & .102 & & \\
& Total & 5.931 & 46 & & & \\
\hline EENG & Between Groups & 1.540 & 5 & .308 & 3.512 & .010 \\
& Within Groups & 3.596 & 41 & .088 & & \\
& Total & 5.136 & 46 & & & \\
\hline UTSTRUE & Between Groups & 1.116 & 5 & .223 & 15.065 & .000 \\
& Within Groups & .607 & 41 & .015 & & \\
& Total & 1.723 & 46 & & & .000 \\
\hline UTSENG & Between Groups & .514 & 5 & .103 & 16.010 & \\
& Within Groups & .263 & 41 & .006 & & \\
& Total & .778 & 46 & & & \\
\hline
\end{tabular}

For UTS $_{\text {true }}$ and UTS $_{\text {eng }}$ Tukey's post hoc test showed there was no significant difference between groups $2,3,4,5$, and 6 . However, these groups were all significantly different from group 1, as shown in Tables 20 and 21. 
Table 18. Tukey post hoc test for the True Young's modulus $\left(E_{\text {true }}\right)$ of Quatromer

\section{ETRUE}

Tukey HSD ${ }^{3 . b}$
\begin{tabular}{|l|r|c|}
\hline & & $\begin{array}{c}\text { Subset } \\
\text { for alpha } \\
=.05\end{array}$ \\
\cline { 2 - 3 } TEST & $\mathrm{N}$ & 1 \\
\hline 1 & 22 & 3.8764 \\
4 & 5 & 4.1000 \\
2 & 5 & 4.1576 \\
3 & 5 & 4.1680 \\
5 & 5 & 4.3000 \\
6 & 5 & 4.4120 \\
Sig. & & .070 \\
\hline
\end{tabular}

Means for groups in homogeneous subsets are displayed.

a. Uses Harmonic Mean Sample Size $=5.739$.

b. The group sizes are unequal. The harmonic mean of the group sizes is used. Type I error levels are not guaranteed.

Table 19. Tukey post hoc test for the Engineering Young's modulus $\left(\mathrm{E}_{\mathrm{eng}}\right)$ of Quatromer

\section{EENG}

Tukey HSD ${ }^{a, b}$
\begin{tabular}{|l|r|c|}
\hline & & $\begin{array}{c}\text { Subset } \\
\text { for alpha } \\
=.05\end{array}$ \\
\cline { 3 - 3 } TEST & $N$ & 1 \\
\hline 1 & 22 & 3.6082 \\
4 & 5 & 3.8040 \\
2 & 5 & 3.8747 \\
3 & 5 & 3.8820 \\
5 & 5 & 4.0020 \\
6 & 5 & 4.1100 \\
Sig. & & .066 \\
\hline
\end{tabular}

Means for groups in homogeneous subsets are displayed.

a. Uses Harmonic Mean Sample Size $=5.739$.

b. The group sizes are unequal. The harmonic mean of the group sizes is used. Type l error levels are not guaranteed. 
Table 20. Tukey post hoc test for the True Ultimate Tensile Stress ( UTS $_{\text {true }}$ ) of Quatromer

UTSTRUE

Tukey HSD ${ }^{\text {a.b }}$
\begin{tabular}{|l|r|c|c|}
\hline \multirow{2}{*}{ TEST } & N & \multicolumn{2}{|c|}{ Subset for alpha $=.05$} \\
\cline { 3 - 4 } & 22 & 1.4277 & 2 \\
\hline 1 & 5 & & 1.7000 \\
4 & 5 & & 1.7116 \\
2 & 5 & & 1.7282 \\
6 & 5 & & 1.7600 \\
5 & 5 & & 1.7700 \\
Sig. & & 1.000 & .923 \\
\hline
\end{tabular}

Means for groups in homogeneous subsets are displayed.

a. Uses Harmonic Mean Sample Size $=5.739$.

b. The group sizes are unequal. The harmonic mean of the group sizes is used. Type I error levels are not guaranteed

Table 21. Tukey post hoc test for the Engineering Ultimate Tensile Stress $\left(\mathrm{UTS}_{\text {eng }}\right)$ of Quatromer

UTSENG

Tukey HSD ${ }^{a, b}$
\begin{tabular}{|l|r|c|c|}
\hline & & \multicolumn{2}{|c|}{ Subset for alpha $=.05$} \\
\cline { 3 - 4 } TEST & $N$ & 1 & 2 \\
\hline 1 & 22 & .9523 & \\
3 & 5 & & 1.1380 \\
4 & 5 & & 1.1450 \\
2 & 5 & & 1.1587 \\
6 & 5 & & 1.1760 \\
5 & 5 & & 1.1840 \\
Sig. & & 1.000 & 924 \\
\hline
\end{tabular}

Means for groups in homogeneous subsets are displayed.

a. Uses Harmonic Mean Sample Size $=5.739$.

b. The group sizes are unequal. The harmonic mean of the group sizes is used. Type I error levels are not guaranteed

For Q + 12 PP fibers, a One-way ANOVA was performed to compare differences between groups in the following parameters: Ultimate Strain (US), True Young's 
modulus ( $\left.E_{\text {true }}\right)$, Engineering Young's modulus $\left(E_{\text {eng }}\right)$, True Ultimate Tensile Stress (UTS true $_{\text {, }}$, and Engineering Ultimate Tensile Stress $\left(\mathrm{UTS}_{\text {eng }}\right)$. Results are presented in Table 22. This table shows there is no significant difference between groups for each of the parameters.

A Tukey post hoc test was performed for each of the parameters in order to find out the differences within each group. There was no significant difference between any of the groups for the different parameters. Results of the Tukey post hoc test are shown in Tables 23, 24, 25, 26 and 27. A summary of the bending fatigue test is shown in Table 28, where "W" means there is a weak significant difference between the fatigued specimens and the control group, "-“ means significant different between the fatigued specimens and the control group, and " $\mathrm{N}$ " means there is no significant difference between the fatigued specimens and the control group.

Table 22. One-way ANOVA results for all Quatromer + 12 PP Fibers groups

\begin{tabular}{|ll|r|r|r|r|r|}
\hline & \multicolumn{1}{c|}{$\begin{array}{c}\text { Sum of } \\
\text { Squares }\end{array}$} & df & Mean Square & \multicolumn{1}{c|}{$F$} & Sig. \\
\hline US & Getween Groups & .008 & 5 & .002 & 1.812 & .143 \\
& Within Groups & .025 & 28 & .001 & & \\
& Total & .033 & 33 & & & \\
\hline ETRUE & Between Groups & 74.727 & 5 & 14.945 & 1.077 & .395 \\
& Within Groups & 388.726 & 28 & 13.883 & & \\
& Total & 463.453 & 33 & & & \\
\hline UTSTRUE & Between Groups & 5.095 & 5 & 1.019 & 1.047 & .410 \\
& Within Groups & 27.250 & 28 & .973 & & \\
& Total & 32.345 & 33 & & & \\
\hline EENG & Between Groups & 64.839 & 5 & 12.968 & 1.079 & .394 \\
& Within Groups & 336.665 & 28 & 12.024 & & \\
& Total & 401.504 & 33 & & & .588 \\
& Between Groups & 1.760 & 5 & .352 & .757 & \\
\hline UTSENG & 13.023 & 28 & .465 & & \\
& Within Groups & 14.783 & 33 & & & \\
& Total & &
\end{tabular}


Table 23. Ultimate Strain (US) Quatromer + 12 PP Fibers

US

Tukey HSD ${ }^{a, b}$
\begin{tabular}{|l|r|r|}
\hline & & $\begin{array}{c}\text { Subset } \\
\text { for alpha } \\
=.05\end{array}$ \\
\cline { 3 - 4 } TEST & $\mathrm{N}$ & 1 \\
\hline 1 & 10 & .30316 \\
2 & 4 & .32250 \\
5 & 5 & .33600 \\
6 & 5 & .33800 \\
3 & 5 & .33800 \\
4 & 5 & .34000 \\
Sig. & & .378 \\
\hline
\end{tabular}

Means for groups in homogeneous subsets are displayed

a. Uses Harmonic Mean Sample Size $=5.217$.

b. The group sizes are unequal. The harmonic mean of the group sizes is used. Type I error levels are not guaranteed.

Table 24. True Young's Modulus $\left(E_{\text {true }}\right)$ for Quatromer + 12 PP Fibers

\section{ETRUE}

Tukey HSD ${ }^{a, b}$

\begin{tabular}{|l|r|c|}
\hline \multirow{2}{*}{ TEST } & N & $\begin{array}{c}\text { Subset } \\
\text { for alpha } \\
=.05\end{array}$ \\
\cline { 3 - 3 } & & 1 \\
\hline 3 & 5 & 41.7080 \\
4 & 5 & 41.8660 \\
1 & 10 & 42.8038 \\
2 & 4 & 44.7225 \\
5 & 5 & 44.8180 \\
6 & 5 & 45.7740 \\
Sig. & & .505 \\
\hline
\end{tabular}

Means for groups in homogeneous subsets are displayed.

a. Uses Harmonic Mean Sample Size $=5.217$.

b. The group sizes are unequal. The harmonic mean of the group sizes is used. Type / error levels are not guaranteed. 
Table 25. True Ultimate Tensile Stress $\left(\mathrm{UTS}_{\text {true }}\right)$ for Quatromer +12 PP Fibers

\section{UTSTRUE}

Tukey HSD ${ }^{a, b}$
\begin{tabular}{|l|r|c|}
\hline & & $\begin{array}{c}\text { Subset } \\
\text { for alpha } \\
=.05\end{array}$ \\
\cline { 3 - 3 } TEST & N & 1 \\
\hline 1 & 10 & 9.7357 \\
4 & 5 & 10.0800 \\
2 & 4 & 10.2175 \\
3 & 5 & 10.2400 \\
5 & 5 & 10.7300 \\
6 & 5 & 10.7400 \\
Sig. & & .578 \\
\hline
\end{tabular}

Means for groups in homogeneous subsets are displayed.

a. Uses Harmonic Mean Sample Size $=5.217$.

b. The group sizes are unequal. The harmonic mean of the group sizes is used. Type I error levels are not guaranteed.

Table 26. True Young's Modulus ( $E_{\text {eng }}$ ) for Quatromer + 12 PP Fibers

\section{EENG}

Tukey HSD
\begin{tabular}{|l|r|c|}
\hline & & $\begin{array}{c}\text { Subset } \\
\text { for alpha } \\
=.05\end{array}$ \\
\cline { 2 - 3 } TEST & $N$ & 1 \\
\hline 3 & 5 & 38.8280 \\
4 & 5 & 38.9680 \\
1 & 10 & 39.8362 \\
2 & 4 & 41.6300 \\
5 & 5 & 41.7180 \\
6 & 5 & 42.6100 \\
Sig. & & .505 \\
\hline
\end{tabular}

Means for groups in homogeneous subsets are displayed.

a. Uses Harmonic Mean Sample Size $=5.217$.

b. The group sizes are unequal. The harmonic mean of the group sizes is used. Type l error levels are not guaranteed. 


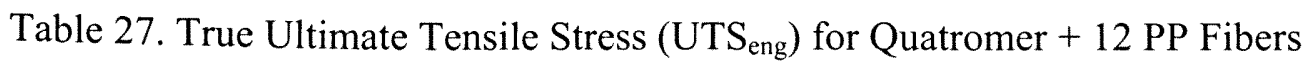

\section{UTSENG}

Tukey HSD ${ }^{a, b}$
\begin{tabular}{|l|r|c|}
\hline & & $\begin{array}{c}\text { Subset } \\
\text { for alpha } \\
=.05\end{array}$ \\
\cline { 3 - 4 } TEST & $N$ & 1 \\
\hline 1 & 10 & 7.4659 \\
4 & 5 & 7.5540 \\
3 & 5 & 7.6460 \\
2 & 4 & 7.7125 \\
6 & 5 & 8.0200 \\
5 & 5 & 8.0400 \\
Sig. & & .750 \\
\hline
\end{tabular}

Means for groups in homogeneous subsets are displayed.

a. Uses Harmonic Mean Sample Size $=5.217$.

b. The group sizes are unequal. The harmonic mean of the group sizes is used. Type I error levels are not guaranteed.

Table 28. Summary of the bending fatigue test

\begin{tabular}{|c|c|c|c|c|c|c|}
\hline \multirow{2}{*}{ Material } & \multirow{2}{*}{ Parameter } & \multicolumn{5}{|c|}{ Number of Cycles (millions) } \\
\hline & & 7 & 16 & 57.5 & 79 & 89 \\
\hline \multirow{4}{*}{$\mathbf{Q}$} & $\mathrm{E}_{\text {true }}$ & W & W & W & W & W \\
\hline & $E_{\text {eng }}$ & W & W & W & W & W \\
\hline & UTS $_{\text {true }}$ & - & - & - & - & - \\
\hline & UTS $_{\text {eng }}$ & - & - & - & - & - \\
\hline \multirow{5}{*}{$Q+12$ PP fibers } & US & $N$ & $N$ & $N$ & $N$ & $N$ \\
\hline & $E_{\text {true }}$ & $N$ & $N$ & $N$ & $N$ & $\mathrm{~N}$ \\
\hline & $E_{\text {eng }}$ & $N$ & $N$ & $N$ & $N$ & $N$ \\
\hline & $\mathrm{UTS}_{\text {true }}$ & $N$ & $N$ & $N$ & $N$ & $N$ \\
\hline & $\mathrm{UTS}_{\mathrm{eng}}$ & $\mathrm{N}$ & $N$ & $N$ & $N$ & $N$ \\
\hline
\end{tabular}




\section{DISCUSSION}

The possibility of using a Quatromer/PP fiber composite as a heart valve material was explored. The mechanical properties of PU, Quatromer, PP fibers, and a Quatromer/PP composite were determined and compared. Results of both static and dynamic mechanical tests on each of the aforementioned materials support the conclusion that the Quatromer/PP composite is a suitable material for use as a heart valve.

Dynamic tests included tension-tension and bending fatigue tests. It has been shown that failure of bioprosthetic and synthetic valves occurs as a consequence of high tensile and bending stresses acting on the leaflet during opening and closing (Chetta and Lloyd 1980; Bernacca, Mackay et al. 1995; Fann and Miller 1996; Bernacca, O'Connor et al. 2002).

Results of the tension-tension fatigue test indicate that the Quatromer/PP composite's fatigue life is statistically greater than that of the PU. PU had a greater fatigue life than Quatromer at the same stress levels. Our first educated guess was to reinforce the Quatromer with 3 PP fibers. Tensile tests were performed for this material followed by a tension-tension fatigue test. When Quatromer was embedded with 3 PP fibers its fatigue life increased, but was still lower than PU at the same stress levels. Therefore, we decided to reinforce the Quatromer with 12 PP fibers. This was number of fibers was estimated from the ultimate tensile force of PU (the material we are comparing against the Quatromer/PP composite) at $0.5 \mathrm{~mm} / \mathrm{mm}$, which was $3.3 \mathrm{~N}$. We would need 11 fibers to reach this amount of force at a strain of $0.1 \mathrm{~mm} / \mathrm{mm}$ (a force of $0.3 \mathrm{~N}$ is needed to strain $1 \mathrm{PP}$ fiber this amount). In order to simplify the manufacture process, we 
decided to reinforce the Quatromer with 12 PP fibers. It is desired not to strain the fibers not more than $0.1 \mathrm{~mm} / \mathrm{mm}$. So, once Quatromer was embedded with 12 PP fibers this composite showed a greater fatigue life than PU. Therefore, varying the number of fibers embedded in the Quatromer may result in reaching the desired dynamic mechanical properties.

It is worth noting that in vitro fatigue studies have not yet been done with the Quatromer as a heart valve material. Studies such as those done by (Chetta and Lloyd 1980; Jansen and Reul 1992; Bernacca, Mackay et al. 1995; Bernacca, O'Connor et al. 2002) have tested heart valves made of PU. Jensen et al. (Jansen and Reul 1992) have found that these heart valves may achieve up to 400 million cycles. This number of cycles represents up to 11 years of fatigue life as number of beats (physiological average of $70 \mathrm{bpm}$ ). In our tensile fatigue test, the $\mathrm{Q}+12 \mathrm{PP}$ fibers composite showed an endurance limit $66 \%$ greater than PU when tested under the same conditions up to 350 million cycles. This represents 10 years of fatigue life (physiological rate of $70 \mathrm{bpm}$ ). Taking this into consideration we might expect a heart valve made of the same vol $\%$ of the $\mathrm{Q}+12$ PP fibers composite to have a greater fatigue life than one manufactured of PU. Recent research (Bernacca, Mackay et al. 1995; Wheatley, Raco et al. 2000) have found polyurethane heart valves start long term deterioration after 4 years of life due to biochemical events.

The bending did not significantly affect the $\mathrm{Q}+12$ PP fibers composite. After the bending fatigue test was performed on this material its tensile properties (US, $E_{\text {true }}, E_{\text {eng, }}$, UTS $_{\text {true, }}$ and $\mathrm{UTS}_{\text {eng }}$ ) were measured. None of them were significantly affected by the cyclic bending. Nevertheless, this was not the case for Quatromer. $E_{\text {true }}$ and $E_{\text {eng }}$ were 
weakly affected by the cyclic bending, while $\mathrm{UTS}_{\text {true, }}$, and $\mathrm{UTS}_{\text {eng }}$ were significantly affected by this test, indicating the material was weakened by the procedure. Thus, once the Quatromer is embedded with 12 PP fibers it is not affected in the long term by cyclic bending stresses. It is worth noting the bending fatigue test was performed up to 89 million cycles. This number of cycles represents up to 2.5 years of fatigue life as number of beats (physiological average of $70 \mathrm{bpm}$ ).

Static tests included the tensile test, Poisson's ratio test, pull-out strength test, and the determination of the viscoelastic properties. Tensile tests show that $Q+12$ PP fibers had higher tensile properties when compared to PU. Even though the tensile properties of Quatromer by itself are lower than the PU that was tested, it is possible to enhance its tensile properties with embedded PP fibers. Quatromer embedded with 3 PP fibers had a Young's modulus in the same range as PU. When Quatromer was reinforced with $12 \mathrm{PP}$ fibers its Young's modulus was more than two times greater than for the PU. This shows that varying the number of fibers embedded in the matrix may modify the tensile properties of the composite. This could prove to be very useful in reaching the desired static mechanical properties. After showing that the rule of mixture may be applied to the materials tested, it is now possible and useful to extend it to a different number of fibers than 3 and 12. Despite the fact that the rule of mixture did not coincide exactly, its values are in the range of those measured. Possible reasons for this difference are: the area of the threads was not measured (an average of the value given by the manufacturer was used), measuring error, and interactions between materials.

The load contribution of the fibers to the composite was very high as compared to the Quatromer. For Q + 3 PP fibers the load contribution of the fibers was $73 \%$, while 
for $\mathrm{Q}+12$ PP fibers it was $95 \%$. These are very high values when taking into account that less than $1 \mathrm{vol} \%$ is carrying $70 \%$ or more of the entire load. The high modulus and strength of the fibers are effectively transmitted to the composite as a whole. At the same time, the ductility of the matrix is available to produce a substantially less brittle material than PP itself. By using a Quatromer/PP composite it is possible to manufacture a heart valve with very thin leaflets. This will favor hydrodynamic function.

Results yielded by measurement of Poisson's ratio indicated that the contraction perpendicular to the extension caused by tensile stresses in the Quatromer are in the range of other polymers like polyurethane foam (0.44), Acrylonitrile-butadiene-styrene (ABS) $(0.38-0.42)$

The pull-out test determined the properties of the fiber/matrix interface. In accordance to Penn et al. (1989) (Penn 1989) findings, the relationship between debonding force and embedded length was not linear, but reached a plateau. In our study a level of maximum force was reached at $0.5 \mathrm{~N}$, where any more contact surface would not increase the bonding force. It should be noted that the force never exceeds this value $(0.5 \mathrm{~N})$, which is the tensile strength of these fibers at this gauge length $(24 \mathrm{~mm})$. Moreover, our single fiber pull-out test displayed the same pattern of behavior as the one observed by Marshall et al. (1991) on a $5 \mu \mathrm{m}$ carbon fiber in a simple epoxy based on DGEBRA resin with an amine curing agent (Marshall and Price 1991).

Results obtained from the viscoelastic tests are difficult to interpret at this point. One explanation that could account for these results is concerned with the fact that the fibers embedded in the Quatromer were not as parallel to the surface as expected. During the preparation of the specimens the fibers had a tendency to sag due to the superficial 
tension created by the Quatromer solution on the fibers as it cured. As a consequence, during the creep test, once the specimen started to strain the fibers first must align with the force before they are able to carry the load. This explains why the creep curves had higher initial strain for the $\mathrm{Q}+12$ PP fibers than for the PP fibers and the Quatromer by themselves.

Although this might be the main cause for the misalignment of the fibers, other factors are worth taking into consideration. The fact that it could not be assured the fibers were parallel between each other could be related to this condition. This case was explained in section 4.2. In addition, the creep tests might be indicating that there is slippage between the PP fibers and the Quatromer. Nevertheless, there is also evidence that contradicts this hypothesis. First, visual inspection of the specimens under the microscope indicates that slippage did not occurred. Specimens were inspected in the extremities and the middle portion. If slippage had occurred the fibers would have debonded from the matrix. The same procedures used in the pull-out test to identify the debonded regions in the specimen were used here. No evidence was found supporting the fact that the fibers had debonded. Secondly, after applying the load to the specimen it strained to a value in the range of $10 \%$, as expected. If slippage had occurred, the specimen would have strained much more, see Figure 19a.

If slippage did occur, it could be argued that this might have affected the tensile tests. However, it is worth taking into consideration the manner the specimens were strained, as well as the amount of force placed on them by each test. While on the creep test a force of $3.5 \mathrm{~N}$ was suddenly applied in a step waveform, during the tensile test the specimens were slowly elongated in a ramp waveform at a rate of $5 \mathrm{~mm} / \mathrm{min}$. Hence, in 
testing the possibility of slippage the effect of different amounts and rates of loading forces should be examined.

It may also be possible that although there is no slippage of the Quatromer with reference to the grips, the fibers may be moving inside the Quatromer relative to the grips with no slippage involved. If this was the case then the fibers would be carrying less amount of the tensile load than the normally would, leaving the matrix supporting the extra load. This would also explain the large strain observed in the creep curves of the Q +12 PP fibers specimen. The fact that no debonding was evidenced supports this idea. As well, to strain the specimens $10 \%$ a $3.5 \mathrm{~N}$ load was applied to the $\mathrm{Q}+12$ PP fibers specimen, while a $0.27 \mathrm{~N}$ load was applied to the Quatromer specimens. This load is more than 10 times greater. If the fibers moved relative to the grips then the Quatromer would have mostly supported the load.

The fact that the fibers need to align before they are able to carry any load must be taken into account in the design of the synthetic heart valve. This situation is similar to the arrangement of the collagen fibers in the heart valve. In the natural valve the collagen fibers are tangled and as the load in the aorta increases the collagen bundles align with the force. It must be considered as a three-dimensional network of fibrils, although the predominant fiber direction is parallel to the surface.

Relaxation results for Quatromer and the PP fiber are very noisy. This is due to the fact that the forces measured for these two materials were relatively very low when compared to those of the other two materials. For Quatromer and the PP fiber forces were in the order of $0.25 \mathrm{~N}$ and $0.20 \mathrm{~N}$ respectively. While for $\mathrm{Q}+12 \mathrm{PP}$ fibers and PU, the forces measured were in the orders of $2.7 \mathrm{~N}$ and $1.45 \mathrm{~N}$ respectively. Still, the path in the 
graph can be seen clearly showing Quatromer had the highest amount of recovery in the shortest period of time, followed by $Q+12$ PP fibers and 1 PP fiber. PU had the least amount of recovery between these materials.

Hysteresis tests were performed in order to present the phenomenon when a body that is subjected to cyclic loading has a different stress-strain relationship in the loading process than in the unloading process. Both PU and Quatromer had the least amount of hysteresis. Conversely, the Q +12 PP fibers composite and the PP fibers showed the largest amount of hysteresis. This suggests that the behavior of the composite is governed by the PP fibers. It must be noted that the PP fibers might have suffered plastic deformation. Even though, all the materials were strained $0.15 \mathrm{~mm} / \mathrm{mm}$, this amount is 1 / 2 of the total elongation of the fibers, while it is a smaller fraction of the total elongation of the Quatromer and the PU.

Based on the results shown in Figure 29, which shows the elastic modulus and the internal friction of the Quatromer/PP composite are constant in all the frequencies at physiological range, we can conclude that this composite will behave in a similar fashion for these frequencies. The response of the Quatromer/PP composite to harmonic excitation would not vary in these frequencies.

One of the limitations of our study is the small number of cycles of the bending fatigue test (equivalent to 2.5 years at a physiological rate of $70 \mathrm{bmp}$ ). It could be the case that the $\mathrm{Q}+12$ PP fibers composite might be significantly affected by tensile and bending stresses over a longer period of time. Nevertheless, the number of cycles of our bending test is equivalent to a considerable amount of time. Moreover, the tensiontension fatigue test was performed for an equivalent of 10 years. In addition, although we 
are at the phase of testing the material, it could be important to take into consideration the amount of time synthetic tri-leaflet heart valves should be tested. According to the Food and Drug Administration, mechanical valves should be tested for an equivalent of 15 years $\left(6 \times 10^{8}\right.$ cycles), while tissue valves of all kinds should be tested for an equivalent of five years ( $2 \times 10^{8}$ cycles), (FDA 1994). However, no statement is made on synthetic tri-leaflet heart valves.

Regarding the static tests it is worth noting that the values for the pull-out strength test could have been obtained over a wider range of length. This way, both the rising and plateau regions might be seen more clearly. This would allow us to compare these two regions. Moreover, during this test a cut was made to the specimen, which could be thought to have affected the results of the pull-out test. Nonetheless, it was not possible to see through the specimen where the cut was made during testing. This leads us to believe that the cut did not open when testing. Hence, the cut seemed not to have had a large effect on the pull-out test. More information about the bonding forces between the Quatromer and the PP fibers is needed, including the microstructure of the fracture.

It cannot be assured that the fibers were always embedded in the Quatromer/PP composite in an equidistant fashion. However, this did not seem to affect the results of the static or dynamic tests. The standard deviation of the tensile test was less than $10 \%$, indicating that there was not much variation among specimens.

The actual tests procedures were carried out in air. It could be argued that results for these tests would differ if carried out in a saline solution at the body's average core temperature $\left(98.6^{\circ} \mathrm{F}\right)$. Nevertheless, Quatromer has been proved to resist boiling 
concentrated nitric acid, which suggests that it will perform well in an oxidative and acidic environments such as those found in the body (Pinchuk, Khan et al. 1999).

In choosing the number of fibers to be embedded in the matrix we took into account that we could take advantage of the high strength of the fibers and the good flexibility of the Quatromer. If the material is too stiff then the leaflets would not perform adequately, as they would not be able to open and close as freely. On the other hand, if the material is too soft the leaflets would not be able to sustain the pressure created in the aorta.

Future studies could pursue the optimization of the Quatromer/PP composite by determining the ideal vol \% of fibers in the Quatromer matrix. In order to do so, the mechanical properties of the materials, which were determined in this study, could be entered in a Finite Element Analysis computer model. Also, biaxial tests on the leaflets are necessary to recollect more information about the mechanical properties of the Quatromer/PP composite and to compare with natural valves. This might prove to be crucial for the optimal performance of the valve. Once this is achieved, efforts could be directed towards in vitro fatigue testing of whole heart valves made from the $\mathrm{Q} / \mathrm{PP}$ composite, followed by pre-clinical testing in vivo to allow for the effects of biological factors. 


\section{CONCLUSION}

In the current state of research on heart valve prostheses it is imperative to find a material with improved fatigue life, which would result in less surgical interventions and reduce the risk of death. Up to date, artificial valves are not as reliable and/or durable as healthy natural ones. Of the two commercially available groups of artificial heart valves, mechanical valves, generally show excellent durability, but also require most patients to receive permanent anticoagulant therapy due to thrombotic reactions. While the other group, bioprosthetic valves, exhibit advantages in hemodynamic properties, producing the central flow characteristic of natural valves. However, they also show leaflet stiffening due to mineralization, which results in short fatigue life (usually less than 10 years). A third group, synthetic tri-leaflet valves, is fabricated from biochemically inert synthetic materials. These valves present natural hemodynamics while also having the potential for long-term durability. Unfortunately, they have not been successful to date due to long-term material degradation. Long-term material degradation occurs through a combination of oxidative reactions with blood and the high dynamic tensile and bending stresses borne by the material. It seems reasonable to assume that synthetic flexible membrane trileaflet valves might be improved with a suitable material choice. Moreover, it was postulated that a decrease in tears and perforations might result from fiberreinforced leaflets reducing high stresses on the leaflets.

In our study we explored the possibilities of a new polymer composite to be used as a synthetic trileaflet heart valve material. This composite was made-up of Quatromer embedded with continuous polypropylene fibers due to the known properties of each of 
these two materials. Quatromer has been found to be less likely to degrade in vivo than polyurethane, while the PP fibers were known to have several unique properties that make them especially suited for use as leaflet reinforcement in a synthetic tri-leaflet heart valve. The primary finding of this study was that the Quatromer/PP composite has the potential to be a more suitable material for use in synthetic tri-leaflet heart valves, as compared to PU (the current material of choice for synthetic heart valve prostheses). In order to do so the mechanical properties of the composite were examined and compared to PU. Results of the tension-tension fatigue test indicate that the Quatromer/PP composite's fatigue life is statistically greater than that of the PU. The Quatromer/PP composite had an endurance limit of $2.5 \mathrm{MPa}$. After being cycled at this stress level for 350 million cycles the $\mathrm{Q}+12$ PP fibers specimen did not fail (this number of cycles represents up to 10 years of fatigue life as number of beats at a physiological average of $70 \mathrm{bpm})$. The endurance for PU was above $1.5 \mathrm{MPa}$. The bending fatigue test did not significantly affect the $\mathrm{Q}+12$ PP fibers composite after being stressed for 89 million cycles of pure bending (this number of cycles represents up to 2.5 years of fatigue life as number of beats at a physiological average of $70 \mathrm{bpm}$ ). The tensile test shows that even though the tensile properties of Quatromer by itself are lower than the PU, which was tested, it is possible to enhance its tensile properties with embedded PP fibers. When Quatromer was embedded with $12 \mathrm{PP}$ fibers its Young's modulus was $45.44 \mathrm{MPa}$, more than two times greater than for the PU, which had a Young's modulus of 18.53 MPa. The Poisson's ratio measured for the Quatromer was 0.41. In the pull-out test a level of maximum force was reached at $0.5 \mathrm{~N}$, where any more contact surface would not increase the bonding force. More than $2 \mathrm{~mm}$ of embedded length would not increase the amount 
of bonding force considerably. These results show that the reinforcement of Quatromer with PP fibers improves both its static and dynamic properties as compared to PU.

Based on this conclusion, we recommend the optimization of the Quatromer/PP composite by determining the ideal vol \% of fibers in the Quatromer matrix. This way it would be possible to manufacture a heart valve with very thin leaflets that would improve the hydrodynamic function while being able to sustain the pressure created in the ventricle. An important next step would involve in vitro testing of synthetic heart valves made of this composite, as well as pre-clinical in vivo studies. This is required to prove the fatigue life of the valve under conditions that resemble to a greater extent those in the heart. Also, it would be necessary to measure the performance of the valve and compare it with other valves already available in the market. 


\section{LIST OF REFERENCES}

Amin, P., J. Willie, et al. (1993). "Analysis of the Extractivew and Hydrolytic Behavior of Microthane poly(ester-urethane) Foam by High Pressure Liquid Chromatography." J. Biomed. Mater. Res. 27: 655.

Bernacca, G. M., T. G. Mackay, et al. (1995). "Calcification and fatigue failure in a polyurethane heart value." Biomaterials 16(4): 279-85.

Bernacca, G. M., B. O'Connor, et al. (2002). "Hydrodynamic function of polyurethane prosthetic heart valves: influences of Young's modulus and leaflet thickness." Biomaterials 23(1): 45-50.

Bernacca, G. M. and D. J. Wheatley (1998). "Surface Modification of Polyurethane Heart Valve: Effects on Fatigue Life and Calcification." Int. J. Artif. Organs 21: 814819.

Cannegieter, S. C., F. R. Rosendaal, et al. (1995). "Optimal oral anticoagulant therapy in patients with mechanical heart valves." N Engl J Med 333(1): 11-7.

Chandran, K. B., R. Fatemi, et al. (1989). "In vitro comparison of velocity profiles and turbulent shear distal to polyurethane trileaflet and pericardial prosthetic valves." Artif Organs 13(2): 148-54.

Chetta, G. E. and J. R. Lloyd (1980). "The design, fabrication and evaluation of a trileaflet prosthetic heart valve." J Biomech Eng 102(1): 34-41.

DeWall, R. A., N. Qasim, et al. (2000). "Evolution of mechanical heart valves." The Annals of Thoracic Surgery 69(5): 1612-1621.

Edmunds, J., L. Henry (2001). "Evolution of prosthetic heart valves." American Heart Journal 141(5): 849-55.

Fann, J. I. and D. C. Miller (1996). "Porcine valves: Hancock and Carpentier-Edwards aortic prostheses.” Semin Thorac Cardiovasc Surg 8(3): 259-68.

FDA (1994). Replacement Heart Valve Guidance, Food and Drug Administration.

Fung, Y. C. (1993). Biomechanics: Mechanical Properties of Living Tissues. La Jolla, CA, Springer.

Imamura, E. and M. P. Kaye (1977). "Function of expanded-polytetrafluoroethylene laminated trileaflet valves in animals." Mayo Clin Proc 52(12): 770-5. 
Jansen, J. and H. Reul (1992). “A synthetic three-leaflet valve.” J Med Eng Technol 16(1): 27-33.

Liu, Y. (2002). A Three-Dimensional Model of a Polymer Composite Tri-Leaflet Aortic Valve Using Finite Element Analysis. Biomedical Engineering. Miami, Florida International University: 121.

Lyengar, A. K. S., H. Sugimoto, et al. (2001). "Dynamic in vitro quantification of bioprosthetic heart valve leaflet motion using structured light projection." Ann Biomed Eng 29(11): 963-73.

Mallick, P. K. (1988). "Fiber-Reinforced Composites: materials, manufacturing, and design.".

Mallick, P. K. (1993). Fiber-reinforced composites materials, manufacturing, and design. New York, N.Y., M. Dekker.

Marshall, P. and J. Price (1991). "Fibre/matrix interface property determination." Composites 22(1): 53-57.

Martini, F. (2001). Fundamentals of Anatomy and Physiology. Upper Saddle River, New Jearsy 07458, Prentice Hall, Inc.

Parfeev, V. M., I. V. Grushetskii, et al. (1982). "Mechanical Properties of Elastomers for Artificial Leaflet Heart Valves." Mekhanika Kompozitnykh Materialov 1: 110117.

Penn, L. S. a. L., S. M. (1989). "Interpretation of Experimental Results in the Single Pullout Filament Test." Journal of Composites Technology \& Research 11(1): 23-30.

Pinchuk, L., M. C. Esquivel, et al. (1991). "Corethane, A New Replacement for Poltether Urethanes for Long-Term Implant Applications." Trans, of the 17th Annual Meeting of the Soc. for Biomat., Scottsdale, AZ.: 98.

Pinchuk, L., I. J. Khan, et al. (1999). "Polyisobutylene-based thermoplastic elastomers for ultra long-term implant applications." Proceedings of the Society of Biomaterials.

Pinchuk, L., J. B. Martin, et al. (1988). "The Use of Silicone/polyurethane Graft Polymers as a Means of Eliminating Surface Cracking of Polyurethane Prosthesis." J. Biomed. Mater. Res. 3: 260.

Press, W. H., S. A. Teukolsky, et al. (1988). Numerical Recipes in C. Cambridge. 
Reul, H. (1983). "In-vitro evaluation of artificial Heart Valves." Adv Cardiol Phy 5: 1630.

Sapirstein, J. S. and P. K. Smith (2001). "The "ideal" replacement heart valve." Am Heart J 141(5): 856-60.

Schoephoerster, R., S. Gallocher, et al. (2001). A Novel Trileaflet Synthetic Heart Valve. Advances in Bioengineering.

Schoephoerster, R. T. and K. B. Chandran (1991). "Velocity and turbulence measurements past mitral valve prostheses in a model left ventricle." 24(7): 549-62.

Senthilnathana, V., T. Treasurea, et al. (1999). "Heart valves: which is the best choice?" Cardiovascular Surgery 7(4): 393-397.

Shackelford, J. F. (1996). Introduction to Materials Science for Engineers. Upper Saddle River, New Jersey, Prentice-Hall.

Wheatley, D. J., L. Raco, et al. (2000). "Polyurethane: material for the next generation of heart valve prostheses?" Eur J Cardiothorac Surg 17(4): 440-8.

Zhao, Q., A. K. McNally, et al. (1993). "Human Plasma a2-Macroglobulin Promotes in vitro Oxidative Stress Cracking of Pellethane 2363-80A: In Vivo and In Vitro Correlations." J. Biomed. Mater. Res. 27: 379-389. 